\title{
Integrated Density of States for the Periodic Schrödinger Operator in Dimension Two
}

\author{
Alexander V. Sobolev
}

\section{Introduction}

The objective of the present paper is to study the high energy asymptotics of the density of states $D(\lambda)$ for the Schrödinger operator $\mathrm{L}^{2}\left(\mathbb{R}^{d}\right), d \geq 1$ with a periodic potential $V$ :

$$
H=-\Delta+V .
$$

Here $V$ is a real-valued function periodic with respect to a $d$-dimensional lattice $\Gamma \subset \mathbb{R}^{d}$. Below we denote by $\mathcal{O} \subset \mathbb{R}^{d}$ a standard fundamental domain of the lattice $\Gamma$, and by $\mathcal{O}^{\dagger}$ the fundamental domain of the dual lattice $\Gamma^{\dagger}$. For this operator, as well as for any other elliptic self-adjoint differential operator, the density of states is defined by the formula

$$
D(\lambda)=\lim _{R \rightarrow \infty} \frac{N\left(\lambda ; H_{D}^{(R)}\right)}{R^{d}} .
$$

Here $H_{D}^{(R)}$ is the restriction of $H$ to the cube $[0, R]^{d}$ with the Dirichlet boundary conditions, and $N(\lambda ; \cdot)$ is the counting function of the discrete spectrum of $H_{D}^{(L)}$. The above limit exists for periodic and almost periodic potentials, see [17], [22]. To be precise, the quantity $D(\lambda)$ is called the integrated density of states, but for the sake of brevity we call it simply the density of states. Calculation of the density of states $D_{0}(\lambda)$ for the unperturbed operator $H_{0}=-\Delta$ is an elementary exercise: one easily proves (see, e.g., Proposition 2.4 below) that

$$
D_{0}(\lambda)=\frac{1}{(2 \pi)^{d}} \mathrm{w}_{d} \lambda^{\frac{d}{2}}, \lambda \geq 0, \quad \mathrm{w}_{d}=\frac{\pi^{\frac{d}{2}}}{\Gamma(1+d / 2)},
$$

where $\mathrm{w}_{d}$ is the volume of the unit ball in $\mathbb{R}^{d}$.

For $d=1$ it was shown in [20] (see [19] for the almost periodic case) that the density of states admits a complete asymptotic expansion in the powers of $\lambda^{-1}$, as $\lambda \rightarrow \infty$. On the basis of this result it is natural to conjecture that for general $d \geq 2$ the asymptotics of $D(\lambda)$ exists and has the form

$$
D(\lambda)=D_{0}(\lambda)\left[1+\sum_{j=1}^{N} b_{j} \lambda^{-j}+o\left(\lambda^{-N}\right)\right], \lambda \rightarrow \infty, \forall N .
$$


For $d=1$ the coefficients $b_{j}$ satisfy simple recursive relations, see [20]. Results for the multidimensional Schrödinger operator are much less advanced: relation (1.4) has been proved only with $N=1$ so far. The first formula was found in [21] (see also [22] for an elementary proof) for almost periodic potentials $V$ :

$$
D(\lambda)=D_{0}(\lambda)+O\left(\lambda^{\frac{d}{2}-1}\right), \lambda \rightarrow \infty .
$$

A two-term asymptotics was first established in [6] for $\mathrm{C}^{\infty}$-smooth periodic potentials $V$ :

$$
D(\lambda)=D_{0}(\lambda)\left[1+b_{1} \lambda^{-1}+O\left(\lambda^{-\frac{3}{2}+\epsilon}\right)\right], \forall \epsilon>0, \quad b_{1}=-\frac{d}{2|\mathcal{O}|} \int_{\mathcal{O}} V(\mathbf{x}) d \mathbf{x} .
$$

The proof in [6] relied based on the powerful methods of microlocal analysis. In [8], via an advanced version of perturbation theory for periodic operators, this asymptotics was generalized to the case of the polyharmonic operator $(-\Delta)^{l}+V$, $l>1 / 2$, with an improvement of the remainder estimate:

$$
D\left(\lambda^{l}\right)=D_{0}\left(\lambda^{l}\right)\left[1+b_{1, l} \lambda^{-l}+O\left(\lambda^{\frac{1}{2}-2 l} \ln \lambda\right)\right], \quad b_{1, l}=b_{1} l^{-1} .
$$

A more precise result was obtained in [8] for the Schrödinger operator $-\Delta+V$ for $d=3$ :

$$
D(\lambda)=D_{0}(\lambda)\left[1+b_{1} \lambda^{-1}\right]+\hat{D}+O\left(\lambda^{-\delta}\right),
$$

with some small $\delta>0$ and a constant coefficient $\hat{D}=\hat{D}_{V}$. Recently it was observed in [11] that $\hat{D}=0$, and hence the above formula is consistent with the conjecture (1.4). Note that the density of states was also studied for the magnetic operator $(-i \nabla-\mathbf{a})^{2}+V$ with a periodic magnetic vector-potential a, see [14] and [8]. In [14] it was shown that $D(\lambda)=D_{0}(\lambda)+O\left(\lambda^{(d-2) / 2+\epsilon}\right)$ with an arbitrary $\epsilon>0$.

Justification of the hypothesis (1.4) for large $N$ would be a very hard problem. However, if one assumes that (1.4) holds, then the coefficients $b_{j}$ can be calculated relatively easily. They are obtained in [11] by a standard argument from the asymptotics of the heat kernel of the Schrödinger operator. These coefficients are integrals of certain standard polynomials depending on $V$ and derivatives of $V$. These polynomials are known both in mathematical and physical literature as heat kernel invariants. For recent work on the structure of these polynomials see, e.g., [16], [7]. In particular, this approach gives

$$
b_{2}=\frac{d(d-2)}{8|\mathcal{O}|} \int_{\mathcal{O}} V^{2}(\mathbf{x}) d \mathbf{x},
$$

which implies that $b_{2}=0$ for $d=2$. Moreover, for all even $d$ the coefficients $b_{j}$ satisfy $b_{j}=0$ if $j \geq d / 2+1$.

Our ultimate goal is to justify the conjecture (1.4) with $N=2$ for all dimensions $d \geq 2$. In this paper this is done for $d=2$, while the general case $d \geq 3$ will 
be addressed in a subsequent publication. The main result of the present paper is the formula

$$
D(\lambda)=D_{0}(\lambda)\left(1+b_{1} \lambda^{-1}\right)+O\left(\lambda^{-\frac{6}{5}+\epsilon}\right), \forall \epsilon>0
$$

for the density of states of the Schrödinger operator with $d=2$, see Theorem 2.3.

From the technical point of view the paper is a continuation of [25] where the density of states was studied in the case $d=1$ for elliptic operators of a more general form than the Schrödinger operator. As in [25], our approach is a variant of the "near-similarity" method, which is usually applied in dimension one (see [18], [1] and [12], [13]). The central idea is to reduce the operator $H$ with the help of a suitable similarity transformation, to an operator with constant coefficients. In the present paper the required similarity is implemented by a unitary operator $e^{i \Psi}$, where $\Psi$ is a bounded self-adjoint PDO with the symbol $\psi(\mathbf{x}, \boldsymbol{\xi})$. In contrast to the one-dimensional case considered in [25], for $d \geq 2$ a complete reduction to constant coefficients is not achievable, since instead of a smooth symbol $\psi(\mathbf{x}, \boldsymbol{\xi})$ (which is the case for $d=1$ ) a straightforward application of the method produces a symbol $\psi$ with singularities on a set $\Lambda$ which is a union of hyper-planes $\{\boldsymbol{\xi} \in$ $\left.\mathbb{R}^{d}: \boldsymbol{\theta}(\boldsymbol{\xi}+\boldsymbol{\theta} / 2)=0\right\}$ where $\boldsymbol{\theta} \in \Gamma^{\dagger}$, $\Gamma^{\dagger}$ being the dual lattice. To avoid the singularity, one studies the neighbourhood of $\Lambda$ separately from the region away from $\Lambda$. Outside the set $\Lambda$ the symbol $\psi$ is found as in the case $d=1$, from a series of commutator equations which emerge as a result of the requirement that the new operator should have constant coefficients. Then the density of states for the new operator is found by an elementary calculation. Near the "singular" set $\Lambda$ the operator $H$ is reduced to a "one-dimensional" effective operator of the Schrödinger type with a pseudo-differential perturbation. For this operator the density of states is found using the results of [25].

Although the set $\Lambda$ emerges in a natural way in the context of the PDO calculus, there is also a perturbation-theoretic interpretation. Recall that the eigenvalues of the unperturbed Floquet Hamiltonian $H_{0}(\mathbf{k})$ are given by $\lambda^{(\boldsymbol{\omega})}(\mathbf{k})=(\boldsymbol{\omega}+\mathbf{k})^{2}$, where $\boldsymbol{\omega} \in \Gamma^{\dagger}$ are points of the dual lattice and $\mathbf{k} \in \mathcal{O}^{\dagger}$ is the quasi-momentum. The analysis of these eigenvalues under the perturbation $V$ is dramatically different for $d=1$ and $d \geq 2$. If $d=1$, then the standard perturbation theory yields a complete asymptotic expansion of the eigenvalues. On the contrary, for $d \geq 2$ the unperturbed eigenvalues split in two groups behaving differently under the perturbation $V$, which can be described with the help of the set $\Lambda$. The eigenvalues $\lambda^{(\boldsymbol{\omega})}(\mathbf{k})$ with $\boldsymbol{\omega} \notin \Lambda$ can be more or less completely described by the perturbation theory, see [4], [9]. The eigenvalues with $\boldsymbol{\omega} \in \Lambda$ move by a quantity of order $\|V\|$ under the perturbation $V$. This effect is due to the small divisors arising when the eigenvalues $\lambda^{(\boldsymbol{\omega})}(\mathbf{k})$ get close together. In the relevant literature these exceptional eigenvalues are sometimes called resonant, unstable or singular, see [5], [9]. It was shown in [5], [9] that their behavior can be described by means of some effective one-dimensional Schrödinger operators.

The resonant set presents a major obstacle when studying spectral properties of the periodic Schrödinger operator, and in particular, the asymptotics of the 
density of states $D(\lambda)$. The precision of the asymptotics eventually depends on how well one knows the behavior of the resonant eigenvalues. For instance, estimating their number from above leads to the remainder estimate in (1.6). The more precise result (1.7) requires more thorough study of the set $\Lambda$, see [8]. In the present paper the asymptotics (1.8) is also derived via a detailed analysis of the set $\Lambda$.

Although the study of the density of states is an object of independent interest in its own right, it can be also used to investigate other spectral properties of the Schrödinger operator. One such problem is to justify the Bethe-Sommerfeld conjecture, that is to prove that the number of gaps in the spectrum of $H$ is finite. The conjecture is known to be true for all dimensions $d \geq 2$ under the condition that the lattice $\Gamma$ is rational, see [23]. For general lattices it was justified so far only for dimensions $d=2,3,4$, see [2], [24], [9], [6], [15] and references therein. This result is derived not directly from (1.5) or (1.6), but from the asymptotics of the same type for the so-called generalized density of states, see, e.g., [6] or [15] for definition. The restriction $d \leq 4$ is then dictated by the remainder estimate in this asymptotics. An improved remainder estimate would lead to the inclusion of bigger $d$ 's. Moreover, the justification of the asymptotics with more terms would allow one to increase the dimension even further.

The paper is organized as follows. Section 2 contains the precise definitions of objects studied in the paper, and the statement of the main result (see Theorem 2.3). In Section 3 necessary information on the calculus of periodic PDO's is collected, including their transformations under linear maps. Section 4 describes partitions of PDO's which are central for their reduction to constant coefficients. Section 5 is devoted to the study of the density of states for the model operator, which is based on its decomposition in the invariant subspaces. Their structure is explicitly described in terms of the resonant set $\Lambda$. On each of the invariant subspaces the model operator reduces to a one-dimensional Schrödinger-type operator, which makes possible the application of the asymptotics established in [25]. At the next step, in Section 6, the Schrödinger operator (1.1) is reduced to the model operator with the help of the unitary operator having the form $e^{i \Psi}$ with a suitable PDO $\Psi$. We loosely call this operator a gauge transformation. A further analysis of the model operator leads to the conclusion that its density of states is determined by its constant coefficients part $A^{\circ}$, see Section 7 . The proof of the Main Theorem is completed in Section 8 together with the asymptotic formula for the density of states of the operator $A^{\circ}$. The calculation of an integral featuring in Section 8, is done in the Appendix. We emphasize that although the main result concerns the case $d=2$, we do the calculations for arbitrary dimension $d \geq 2$ whenever possible, indicating the points where the argument requires $d=2$. 


\section{Main result}

\subsection{Classes of PDO's}

Before we define the pseudo-differential operators (PDO's) we introduce first the relevant classes of symbols. Let $\Gamma \in \mathbb{R}^{d}$ be a lattice. Denote by $\mathcal{O}$ its fundamental domain. For example, for $\mathcal{O}$ one can choose a parallelepiped spanned by a basis of $\Gamma$. The dual lattice and its fundamental domain are denoted by $\Gamma^{\dagger}$ and $\mathcal{O}^{\dagger}$ respectively. Sometimes we reflect the dependence on the lattice and write $\mathcal{O}_{\Gamma}$ and $\mathcal{O}_{\Gamma}^{\dagger}$. In particular in the case $\Gamma=(2 \pi \mathbb{Z})^{d}$ one has $\Gamma^{\dagger}=\mathbb{Z}^{d}$ and it is natural to take $\mathcal{O}=[0,2 \pi)^{d}, \mathcal{O}^{\dagger}=[0,1)^{d}$. For any measurable set $\mathcal{C} \subset \mathbb{R}^{d}$ we denote by $|\mathcal{C}|$ or $\operatorname{vol}(\mathcal{C})$ its Lebesgue measure (volume). The volume of the fundamental domain does not depend on its choice, it is called the determinant of the lattice $\Gamma$ and denoted $\mathrm{d}(\Gamma)=|\mathcal{O}|$. By $\mathbf{e}_{1}, \mathbf{e}_{2}, \ldots, \mathbf{e}_{d}$ we denote the standard orthonormal basis in $\mathbb{R}^{d}$.

For any $u \in \mathrm{L}^{2}(\mathcal{O})$ and $f \in \mathrm{L}^{2}\left(\mathbb{R}^{d}\right)$ define the Fourier coefficients and Fourier transform respectively:

$$
\begin{gathered}
\hat{u}(\boldsymbol{\theta})=\frac{1}{\sqrt{\mathrm{d}(\Gamma)}} \int_{\mathcal{O}} e^{-i\langle\boldsymbol{\theta}, \mathbf{x}\rangle} u(\mathbf{x}) d \mathbf{x}, \boldsymbol{\theta} \in \Gamma^{\dagger}, \\
(\mathcal{F} f)(\boldsymbol{\xi})=\frac{1}{(2 \pi)^{\frac{d}{2}}} \int_{\mathbb{R}^{d}} e^{-i\langle\boldsymbol{\xi}, \mathbf{x}\rangle} f(\mathbf{x}) d \mathbf{x}, \boldsymbol{\xi} \in \mathbb{R}^{d} .
\end{gathered}
$$

Let us now define the periodic symbols and PDO's associated with them. Let $b=b(\mathbf{x}, \boldsymbol{\xi}), \mathbf{x}, \boldsymbol{\xi} \in \mathbb{R}^{d}$, be a $\Gamma$-periodic complex-valued function, i.e.,

$$
b(\mathbf{x}+\boldsymbol{\gamma}, \boldsymbol{\xi})=b(\mathbf{x}, \boldsymbol{\xi}), \forall \boldsymbol{\gamma} \in \Gamma
$$

Let $w: \mathbb{R}^{d} \rightarrow \mathbb{R}$ be a locally bounded function such that $w(\boldsymbol{\xi}) \geq 1, \forall \boldsymbol{\xi} \in \mathbb{R}^{d}$ and

$$
w(\boldsymbol{\xi}+\boldsymbol{\eta}) \leq C w(\boldsymbol{\xi})\langle\boldsymbol{\eta}\rangle^{\kappa}, \forall \boldsymbol{\xi}, \boldsymbol{\eta} \in \mathbb{R}^{d},
$$

for some $\kappa \geq 0$. We say that the symbol $b$ belongs to the class $\mathbf{S}_{\alpha}=\mathbf{S}_{\alpha}(w)=$ $\mathbf{S}_{\alpha}(w, \Gamma), \alpha \in \mathbb{R}$, if for any $l \geq 0$ and any non-negative $s \in \mathbb{Z}$ the condition

$$
|b|_{l, s}^{(\alpha)}=\max _{p \leq l} \max _{|\mathbf{s}| \leq s} \sup _{\boldsymbol{\xi}, \boldsymbol{\theta}}\langle\boldsymbol{\theta}\rangle^{p} w(\boldsymbol{\xi})^{-\alpha+|\mathbf{s}|}\left|\mathbf{D}_{\boldsymbol{\xi}}^{\mathbf{s}} \hat{b}(\boldsymbol{\theta}, \boldsymbol{\xi})\right|<\infty
$$

is fulfilled. If necessary, we reflect the dependence of this norm on the weight $w$ and write $|b|_{l, s ; w}^{(\alpha)}$. Here we have used the standard notation $\langle\mathbf{t}\rangle=\sqrt{1+|\mathbf{t}|^{2}}, \forall \mathbf{t} \in \mathbb{R}^{d}$. Also, for any $\mathbf{s} \in \mathbb{Z}^{d}$ we denote $|\mathbf{s}|=s_{1}+s_{2}+\cdots+s_{d}$. We mainly use two types of classes $\mathbf{S}_{\alpha}$ : either with the weight $w(\boldsymbol{\xi})=\langle\boldsymbol{\xi}\rangle$, which satisfies $(2.1)$ for $\kappa=1$, or with a weight $w(\boldsymbol{\xi})=L$ where a constant $L$ is chosen in a convenient way. Note that $\mathbf{S}_{\alpha}$ is an increasing function of $\alpha$, i.e., $\mathbf{S}_{\alpha} \subset \mathbf{S}_{\beta}$ for $\alpha<\beta$. For later 
reference write the following convenient bounds that follow from Definition (2.2) and property (2.1):

$$
\begin{gathered}
\left|\mathbf{D}_{\xi}^{\mathbf{s}} \hat{b}(\boldsymbol{\theta}, \boldsymbol{\xi})\right| \leq|b|_{l, s}^{(\alpha)}\langle\boldsymbol{\theta}\rangle^{-l} w(\boldsymbol{\xi})^{\alpha-s}, \\
\left|\mathbf{D}_{\xi}^{\mathbf{s}} \hat{b}(\boldsymbol{\theta}, \boldsymbol{\xi}+\boldsymbol{\eta})-\mathbf{D}_{\boldsymbol{\xi}}^{\mathbf{s}} \hat{b}(\boldsymbol{\theta}, \boldsymbol{\xi})\right| \leq C|b|_{l, s+1}^{(\alpha)}\langle\boldsymbol{\theta}\rangle^{-l} w(\boldsymbol{\xi})^{\alpha-s-1}\langle\boldsymbol{\eta}\rangle^{\kappa|\alpha-s-1|}|\boldsymbol{\eta}|, \quad s=|\mathbf{s}|,
\end{gathered}
$$

with a constant $C$ depending only on $\alpha, s$. We introduce a separate notation for the set $\mathbf{P}_{\alpha}=\mathbf{P}_{\alpha}(w, \Gamma) \supset \mathbf{S}_{\alpha}$ of symbols $b$ such that $|b|_{l, 0}^{(\alpha)}<\infty$ for all $l \geq 0$. Periodic functions $V \in \mathrm{C}^{\infty}\left(\mathbb{R}^{d}\right)$ can be also viewed as symbols from $\mathbf{P}_{0}$. For such functions $|V|_{l, s}^{(0)}=|V|_{l, 0}^{(0)}$ for any $s$.

Now we define the PDO $\mathrm{Op}(b)$ in the usual way:

$$
\mathrm{Op}(b) u(\mathbf{x})=\frac{1}{(2 \pi)^{\frac{d}{2}}} \int b(\mathbf{x}, \boldsymbol{\xi}) e^{i\langle\boldsymbol{\xi}, \mathbf{x}\rangle}(\mathcal{F} u)(\boldsymbol{\xi}) d \boldsymbol{\xi},
$$

the integral being taken over $\mathbb{R}^{d}$. Under the condition $b \in \mathbf{P}_{\alpha}$ the integral in the r.h.s. is clearly finite for any $u$ from the class $\mathrm{B}\left(\mathbb{R}^{d}\right)$ of functions such that their Fourier transforms decay faster than any power of $\boldsymbol{\xi}$, that is

$$
\mathrm{B}\left(\mathbb{R}^{d}\right)=\left\{u: \sup _{\boldsymbol{\xi}}\langle\boldsymbol{\xi}\rangle^{l}|(\mathcal{F} u)(\boldsymbol{\xi})|<\infty, \forall l>0\right\} .
$$

In particular, $\mathrm{Op}(b)$ is well defined for $u$ in the Schwarz class $\mathbf{S}\left(\mathbb{R}^{d}\right)$. Moreover, the condition $b \in \mathbf{P}_{0}$ guarantees the boundedness of $\mathrm{Op}(b)$ in $\mathrm{L}^{2}\left(\mathbb{R}^{d}\right)$, see Proposition 3.1. Unless otherwise stated, from now on $S\left(\mathbb{R}^{d}\right)$ is taken as a natural domain for all PDO's at hand, although sometimes we need to consider $\mathrm{Op}(b)$ on functions from the bigger class $\mathrm{B}\left(\mathbb{R}^{d}\right)$ as well. Observe that the operator $\mathrm{Op}(b)$ is symmetric if its symbol satisfies the condition

$$
\hat{b}(\boldsymbol{\theta}, \boldsymbol{\xi})=\overline{\hat{b}(-\boldsymbol{\theta}, \boldsymbol{\xi}+\boldsymbol{\theta})} .
$$

We shall call such symbols symmetric.

Note that $\mathbf{S}_{\alpha}(L)=\mathbf{S}_{\beta}(L)$ for any $\alpha, \beta \in \mathbb{R}$ and

$$
|b|_{l, s}^{(\alpha)}=L^{\beta-\alpha}|b|_{l, s}^{(\beta)} .
$$

In fact, the introduction of different notation for the same class is done here to reflect the dependence on the parameter $L$.

Throughout the entire paper we adopt the following convention. An estimate (or an assertion) is said to be uniform in a symbol $b \in \mathbf{S}_{\alpha}$ (resp. $\mathbf{P}_{\alpha}$ ) if the constants in the estimate (or assertion) at hand depend only on the constants $C_{l, s}$ (resp. $C_{l, 0}$ ) in the bounds $|b|_{l, s}^{(\alpha)} \leq C_{l, s}$.

As was indicated in the introduction, our ultimate goal is to study the density of states of the Schrödinger operator $H=H_{0}+V$ in $\mathcal{H}=\mathrm{L}^{2}\left(\mathbb{R}^{d}\right)$ with $H_{0}=-\Delta$ 
and a smooth real-valued periodic potential $V$. However, some general definitions are more natural to give for more general operators. For these purposes it is not even necessary to assume that $H_{0}=-\Delta$, but it would be sufficient to suppose that $H_{0}=\mathrm{Op}\left(h_{0}\right)$ with $h_{0}(\boldsymbol{\xi})=|\mathbf{F} \boldsymbol{\xi}|^{m}, m>0$, where $\mathbf{F}$ is a non-degenerate $d \times d$ matrix. Also, the perturbation is allowed to be an arbitrary PDO of order lower than $H_{0}$. Another reason of considering more general operators is methodological: a number of intermediate results requires the use of such PDO's. Precisely, we consider the operator

$$
\left\{\begin{array}{l}
H=\mathrm{Op}(h), h(\mathbf{x}, \boldsymbol{\xi})=h_{0}(\boldsymbol{\xi})+b(\mathbf{x}, \boldsymbol{\xi}), \\
h_{0}(\boldsymbol{\xi})=|\mathbf{F} \boldsymbol{\xi}|^{m}, \quad b \in \mathbf{P}_{\alpha}(\langle\boldsymbol{\xi}\rangle), \quad \alpha<m,
\end{array}\right.
$$

with a symmetric symbol $b$. The operator $\mathrm{Op}(b)$ is $H_{0}$-bounded with an arbitrarily small relative bound. Thus $H$ is self-adjoint on the domain $D(H)=D\left(H_{0}\right)=$ $\mathrm{H}^{m}\left(\mathbb{R}^{d}\right)$. Sometimes we call symbols (PDO's) of this type elliptic symbols (PDO's) of order $m$. In this paper we do not need to consider more general elliptic symbols. Due to the $\Gamma$-periodicity of the symbol $b$, the operator $H$ commutes with the shifts along the lattice vectors, i.e.,

$$
H \mathcal{T}_{\gamma}=\mathcal{T}_{\gamma} H, \gamma \in \Gamma .
$$

with $\left(\mathcal{T}_{\boldsymbol{\gamma}} u\right)(\mathbf{x})=u(\mathbf{x}+\boldsymbol{\gamma})$. This allows us to use the Floquet decomposition.

\subsection{Floquet decomposition}

We identify the space $\mathcal{H}=\mathrm{L}^{2}\left(\mathbb{R}^{d}\right)$ with the direct integral

$$
\mathfrak{G}=\int_{\mathcal{O}^{\dagger}} \mathfrak{H} d \mathbf{k}, \mathfrak{H}=\mathrm{L}^{2}(\mathcal{O})
$$

This identification is implemented by the Gelfand transform

$$
(U u)(\mathbf{x}, \mathbf{k})=\frac{1}{\sqrt{\mathrm{d}\left(\Gamma^{\dagger}\right)}} e^{-i\langle\mathbf{k}, \mathbf{x}\rangle} \sum_{\gamma \in \Gamma} e^{-i\langle\mathbf{k}, \gamma\rangle} u(\mathbf{x}+\gamma), \mathbf{k} \in \mathcal{O}^{\dagger}
$$

which is initially defined on $u \in \mathbf{S}\left(\mathbb{R}^{d}\right)$ and extends by continuity to a unitary mapping from $\mathcal{H}$ onto $\mathfrak{G}$. In terms of the Fourier transform the Gelfand transform is defined as follows: $\widehat{(U u)}(\boldsymbol{\theta}, \mathbf{k})=(\mathcal{F} u)(\boldsymbol{\theta}+\mathbf{k}), \boldsymbol{\theta} \in \Gamma^{\dagger}$. The unitary operator $U$ reduces $\mathcal{T}_{\boldsymbol{\gamma}}$ to the diagonal form:

$$
\left(U \mathcal{T}_{\gamma} U^{-1} f\right)(\cdot, \mathbf{k})=e^{i\langle\mathbf{k}, \boldsymbol{\gamma}\rangle} f(\cdot, \mathbf{k}), \forall \gamma \in \Gamma .
$$

Let us consider a self-adjoint operator $A$ in $\mathcal{H}$ which commutes with $\mathcal{T}_{\gamma}$ for all $\gamma \in \Gamma$, i.e., $A \mathcal{T}_{\gamma}=\mathcal{T}_{\gamma} A$. We call such operators $\Gamma$-periodic or simply periodic. 
Then $A$ is partially diagonalized by $U$ (see [17]), that is, there exists a measurable family of self-adjoint operators (fibres) $A(\mathbf{k})$ acting in $\mathfrak{H}$, such that

$$
U A U^{*}=\int_{\mathcal{O}^{\dagger}} A(\mathbf{k}) d \mathbf{k} .
$$

It is easy to show that any periodic symmetric operator $T$, which is $A$-bounded with relative bound $\epsilon<1$, can be also decomposed into a measurable set of fibers $T(\mathbf{k})$ in the sense that

$$
(U T f)(\cdot, \mathbf{k})=T(\mathbf{k})(U f)(\cdot, \mathbf{k}), \text { a.e. } \mathbf{k} \in \mathcal{O}^{\dagger},
$$

for all $f \in D(A)$. Moreover, the fibers $T(\mathbf{k})$ are $A(\mathbf{k})$-bounded with the bound $\epsilon$, and if $T$ is symmetric, then the operator $A(\mathbf{k})+T(\mathbf{k})$ is self-adjoint on $D(A(\mathbf{k}))$.

Suppose that the operator $A$ (and hence $A(\mathbf{k})$ ) is bounded from below and that the spectrum of each $A(\mathbf{k})$ is discrete. Denote by $\lambda_{j}(A(\mathbf{k})), j=1,2, \ldots$, the eigenvalues of $A(\mathbf{k})$ labelled in the ascending order. Using the min-max principle one easily sees that each $\lambda_{j}(A(\cdot))$ is a measurable function of $\mathbf{k}$. Suppose also that the counting function

$$
N(\lambda, A(\mathbf{k}))=\#\left\{j: \lambda_{j}(A(\mathbf{k})) \leq \lambda\right\}, \lambda \in \mathbb{R},
$$

is bounded as a function of $\mathbf{k} \in \mathcal{O}^{\dagger}$. Then we define the integrated density of states by the formula

$$
D(\lambda)=D(\lambda ; A)=\frac{1}{(2 \pi)^{d}} \int_{\mathcal{O}^{\dagger}} N(\lambda, A(\mathbf{k})) d \mathbf{k} .
$$

This definition makes sense for the operator $A+T$ as well, since $N(\lambda, A(\cdot)+$ $T(\cdot)) \in \mathrm{L}^{\infty}\left(\mathcal{O}^{\dagger}\right)$. Sometimes we need to reflect the dependence of the counting function and density of states on the lattice. In this case we use the notation $N_{\Gamma}(\lambda, A(\mathbf{k})), D_{\Gamma}(\lambda ; A)$.

Let us indicate some elementary general properties of the density of states, following directly from Definition (2.11).

Proposition 2.1 Let $A, A_{1}$ be self-adjoint $\Gamma$-periodic operators as defined above.

(i) The density of states is monotone in $A$, that is, if $A \leq A_{1}$, then $D(\lambda ; A) \geq$ $D\left(\lambda ; A_{1}\right)$.

(ii) The density of states is a unitary invariant. Precisely, for any unitary $\Gamma$ periodic operator $W$ one has $D\left(\lambda ; W^{*} A W\right)=D(\lambda ; A)$.

Proof. The inequality in (i) follows from the inequality $A(\mathbf{k}) \leq A_{1}(\mathbf{k})$, a.a. $\mathbf{k} \in \mathcal{O}^{\dagger}$. The unitary invariance follows from the identity $N(\lambda, A(\mathbf{k}))=N\left(\lambda, W^{*}(\mathbf{k}) A(\mathbf{k})\right.$ $W(\mathbf{k}))$, where $W(\mathbf{k})$ are the fibres of the operator $W$ in the decomposition (2.10). 
If $A=\mathrm{Op}(a)$ with a real-valued symbol $a \in \mathrm{L}_{\text {loc }}^{\infty}\left(\mathbb{R}^{d}\right)$ depending only on $\boldsymbol{\xi}$, then $A(\mathbf{k})$ is a self-adjoint PDO on the torus $\mathbb{T}_{\Gamma}^{d}=\mathbb{R}^{d} / \Gamma$ defined as follows:

$$
A(\mathbf{k}) u(\mathbf{x})=\frac{1}{\sqrt{\mathrm{d}(\Gamma)}} \sum_{\boldsymbol{\gamma}^{\dagger} \in \Gamma^{\dagger}} e^{i\left\langle\boldsymbol{\gamma}^{\dagger}, \mathbf{x}\right\rangle} a\left(\boldsymbol{\gamma}^{\dagger}+\mathbf{k}\right) \hat{u}\left(\boldsymbol{\gamma}^{\dagger}\right) .
$$

If $a(\boldsymbol{\xi}) \rightarrow \infty$ as $|\boldsymbol{\xi}| \rightarrow \infty$, then the spectrum of each $A(\mathbf{k})$ is purely discrete with eigenvalues given by $\lambda^{(\mathbf{m})}(\mathbf{k})=a(\mathbf{m}+\mathbf{k}), \mathbf{m} \in \Gamma^{\dagger}$. Consequently, the number of eigenvalues below each $\lambda \in \mathbb{R}$ is essentially bounded from above uniformly in $\mathbf{k} \in \mathcal{O}^{\dagger}$. If $T$ is a periodic symmetric operator which is $A$-bounded with a bound $\epsilon<1$, then the spectrum of $A(\mathbf{k})+T(\mathbf{k})$ is also purely discrete and the counting function is also bounded uniformly in $\mathbf{k}$.

The above applies to the elliptic operator $H$ defined in (2.8), and thus the quantity $D(\lambda ; H)$ is well defined. In fact, if necessary, one can obtain more information on the operators $H_{0}(\mathbf{k})$ and $H(\mathbf{k})$. Applying the Gelfand transform (2.9) to $\mathrm{Op}(b)$, one finds that, similarly to $A$ considered above, the operator $H(\mathbf{k})$ is a PDO on the torus $\mathbb{T}_{\Gamma}^{d}$ of the form

$$
H(\mathbf{k}) u(\mathbf{x})=\frac{1}{\sqrt{\mathrm{d}(\Gamma)}} \sum_{\boldsymbol{\gamma}^{\dagger} \in \Gamma^{\dagger}} e^{i\left\langle\gamma^{\dagger}, \mathbf{x}\right\rangle} h\left(\mathbf{x}, \gamma^{\dagger}+\mathbf{k}\right) \hat{u}\left(\boldsymbol{\gamma}^{\dagger}\right) .
$$

Moreover, if the symbol $b(\mathbf{x}, \boldsymbol{\xi})$ is smooth in $\boldsymbol{\xi}$, then the family $H(\mathbf{k})$ is smooth in $\mathbf{k}$. Note however that for our purposes we need neither this explicit formula, nor the smoothness property.

Recall that for periodic elliptic differential operators $A$ there is another, equivalent, definition of the density of states given by (1.2). In the case of a pseudo-differential operator the formula (1.2) is not applicable, and we use (2.11) as definition. It is important however to convince oneself that the formula $(2.11)$ preserves the invariance of $D(\lambda)$ with respect to the choice of the lattice in the following sense: if the operator $H$ happens to be periodic with respect to the lattices $\Gamma$ and $\Lambda$, then $D_{\Gamma}(\lambda)=D_{\Lambda}(\lambda)$. To this end we write another formula for $D(\lambda)$ in terms of the spectral projection $E(\lambda)=\chi(H ;(-\infty, \lambda])$ for $H$. Here and everywhere below we denote by $\chi(\cdot ; \mathcal{C})$ the characteristic function of a measurable set $\mathcal{C} \subset \mathbb{R}^{m}$. Denote

$$
T(\lambda, \mathcal{C})=\operatorname{tr}(E(\lambda) \chi(\mathbf{x} ; \mathcal{C})) .
$$

It is easy to show that this trace is finite for any bounded set $\mathcal{C} \subset \mathbb{R}^{d}$.

Theorem 2.2 Let the operator $H$ be as defined in (2.8). Let $D(\lambda ; H)$ be its density of states defined by the formula (2.11). Then

$$
D(\lambda ; H)=\lim _{R \rightarrow \infty} \frac{1}{R^{d}} T\left(\lambda, \mathcal{K}_{R}\right),
$$

where $\mathcal{K}_{R}=[0, R]^{d}$. 
Proof. Let us make a preliminary calculation. Let $B$ be a finite subset of $\Gamma$, and let $\mathcal{C}=\cup_{\gamma \in B}(\gamma+\mathcal{O})$ be the set consisting of $\mathcal{O}$ and its translations by the vectors $\gamma \in B$. Using the invariance of the trace under unitary transformations, rewrite using the Gelfand transform (2.9):

$$
T(\lambda, \mathcal{C})=\operatorname{tr}\left(U E(\lambda) U^{*} U \chi(\cdot, \mathcal{C}) U^{*}\right) .
$$

A straightforward calculation shows that the operator $U \chi U^{*}$ has the form

$$
\begin{aligned}
\left(U \chi U^{*} f\right)(\mathbf{x}, \mathbf{k}) & =\int_{\mathcal{O}^{\dagger}} M\left(\mathbf{x} ; \mathbf{k}-\mathbf{k}^{\prime}\right) f\left(\mathbf{x}, \mathbf{k}^{\prime}\right) d \mathbf{k}^{\prime}, \\
M(\mathbf{x} ; \mathbf{t}) & =\frac{1}{\mathrm{~d}\left(\Gamma^{\dagger}\right)} \sum_{\gamma \in B} e^{-i\langle\mathbf{t}, \mathbf{x}+\gamma\rangle} .
\end{aligned}
$$

To find the trace $T(\lambda, \mathcal{C})$ we use the formula

$$
\operatorname{tr} K=\sum_{n}\left(K \phi_{n}, \phi_{n}\right)
$$

which gives tr $K$ for any trace-class operator $K$ in a Hilbert space as the sum over an arbitrary orthonormal basis $\left\{\phi_{n}\right\}$. Let us take the following orthonormal basis in $\mathfrak{G}$ :

$$
\begin{gathered}
\psi_{\boldsymbol{\gamma}, \boldsymbol{\omega}}(\mathbf{x}, \mathbf{k})=f_{\boldsymbol{\gamma}}(\mathbf{k}) g_{\boldsymbol{\omega}}(\mathbf{x}), \\
f_{\boldsymbol{\gamma}}(\mathbf{k})=\frac{1}{\sqrt{\mathrm{d}\left(\Gamma^{\dagger}\right)}} e^{i\langle\mathbf{k}, \boldsymbol{\gamma}\rangle}, g_{\boldsymbol{\omega}}(\mathbf{x})=\frac{1}{\sqrt{\mathrm{d}(\Gamma)}} e^{i\langle\mathbf{x}, \boldsymbol{\omega}\rangle}, \gamma \in \Gamma, \boldsymbol{\omega} \in \Gamma^{\dagger}
\end{gathered}
$$

Denote $\left(U E(\lambda) U^{*}\right)(\mathbf{k})=E(\lambda, \mathbf{k})$. Then

$$
\begin{aligned}
T(\lambda, \mathcal{C}) & =\sum_{\gamma, \boldsymbol{\omega}} \int_{\mathcal{O}^{\dagger}} \int_{\mathcal{O}^{\dagger}}\left(E(\lambda, \mathbf{k}) M\left(\cdot ; \mathbf{k}-\mathbf{k}^{\prime}\right) g_{\boldsymbol{\omega}}, g_{\boldsymbol{\omega}}\right) f_{\boldsymbol{\gamma}}(\mathbf{k}) \overline{f_{\gamma}\left(\mathbf{k}^{\prime}\right)} d \mathbf{k} d \mathbf{k}^{\prime} \\
& =\sum_{\boldsymbol{\omega}} \int_{\mathcal{O}^{\dagger}}\left(E(\lambda, \mathbf{k}) M(\cdot ; \mathbf{0}) g_{\boldsymbol{\omega}}, g_{\boldsymbol{\omega}}\right) d \mathbf{k} .
\end{aligned}
$$

Note that

$$
M(\mathbf{x} ; \mathbf{0})=\frac{1}{\mathrm{~d}\left(\Gamma^{\dagger}\right)} \sharp\{\gamma \in B\}=\frac{1}{\mathrm{~d}\left(\Gamma^{\dagger}\right) \mathrm{d}(\Gamma)}|\mathcal{C}|=\frac{1}{(2 \pi)^{d}}|\mathcal{C}|,
$$

where $|\mathfrak{C}|$ denotes the Lebesgue measure of $\mathcal{C}$. Consequently

$$
T(\lambda, \mathcal{C})=\frac{1}{(2 \pi)^{d}}|\mathcal{C}| \int_{\mathcal{O}^{\dagger}} \operatorname{tr} E(\lambda, \mathbf{k}) d \mathbf{k}=\frac{1}{(2 \pi)^{d}}|\mathcal{C}| \int_{\mathcal{O}^{\dagger}} N(\lambda, H(\mathbf{k})) d \mathbf{k}=|\mathcal{C}| D(\lambda) .
$$

Now let $\mathcal{C}_{>}\left(\right.$resp. $\left.\mathcal{C}_{<}\right)$be the maximal (resp. minimal) set consisting of the domain $\mathcal{O}$ and its translations by the vectors $\gamma \in \Gamma$, such that $\mathcal{C}_{<} \subset \mathcal{K}_{R} \subset \mathcal{C}_{>}$. Then, clearly,

$$
T\left(\lambda, \mathcal{C}_{<}\right) \leq T\left(\lambda, \mathcal{K}_{R}\right) \leq T\left(\lambda, \mathcal{C}_{>}\right),
$$

and both volumes $\left|\mathcal{C}_{<}\right|$and $\left|\mathcal{C}_{>}\right|$are $R^{d}+o\left(R^{d}\right), R \rightarrow \infty$. Applying (2.12) to $\mathfrak{C}_{<}$ and $\mathcal{C}_{>}$we obtain the required formula. 


\subsection{Main result}

We are now in a position to state the main result of the paper. Let $V$ be a $\Gamma$-periodic function, and let $H=-\Delta+V$. In the main Theorem below we assume that $d=2$. The multidimensional case will be considered in a subsequent publication.

Theorem 2.3 Let $d=2$. Assume that $V \in C^{\infty}\left(\mathbb{R}^{d}\right)$ is $\Gamma$-periodic and that $\hat{V}(\mathbf{0})=0$. Then there is a number $\lambda_{0}=\lambda_{0}(V)>0$ such that

$$
D(\lambda ; H)=\frac{1}{4 \pi} \lambda+O_{\delta}\left(\lambda^{-\frac{6}{5}+\delta}\right), \forall \delta>0,
$$

for all $\lambda \geq \lambda_{0}$. The constant $\lambda_{0}$ and the remainder estimate are uniform in $V$ in the sense that they depend only on the constants in the bounds $|V|_{l, 0}^{(0)} \leq C_{l}$.

The condition $\hat{V}(\mathbf{0})=0$ does not restrict generality, since $\hat{V}(\mathbf{0})$ can be always incorporated into the spectral parameter $\lambda$. This simple argument allows one to deduce the formula (1.8) announced in the introduction, from Theorem 2.3.

\section{4 "Partial" density of states}

Now we define the density of states for PDO's on their invariant subspaces. To this end we need to introduce a class of projection operators. Let $\mathcal{C} \subset \mathbb{R}^{d}$ be a measurable set. Denote by $\chi(\boldsymbol{\xi})=\chi(\boldsymbol{\xi} ; \mathcal{C})$ the characteristic function of $\mathcal{C}$. Then the operator $\mathcal{P}(\mathcal{C})=\chi(\mathbf{D} ; \mathcal{C})=\operatorname{Op}(\chi(\cdot ; \mathfrak{C}))$ is a projection in $\mathcal{H}$ on the subspace $\mathcal{H}(\mathcal{C})=\mathcal{P}(\mathcal{C}) \mathcal{H}$, and the operator $\mathcal{P}(\mathbf{k})=\mathcal{P}(\mathbf{k} ; \mathcal{C})$ is a PDO on the torus with the symbol $\chi\left(\gamma^{\dagger}+\mathbf{k}\right)$.

Suppose that $\mathcal{H}(\mathcal{C})$ is an invariant subspace of the operator $H$ defined in (2.8), that is $\mathcal{P} D(H) \subset D(H)$ and $H \mathcal{P} D(H) \subset \mathcal{H}(\mathcal{C})$. Then the subspace $\mathfrak{H}(\mathbf{k})=\mathcal{P}(\mathbf{k}) \mathfrak{H}$, $\mathbf{k} \in \mathcal{O}^{\dagger}$, is invariant for $H(\mathbf{k})$. Since the spectrum of $H(\mathbf{k})$ is purely discrete, then so is the spectrum of the restriction of $H(\mathbf{k})$ to the subspace $\mathfrak{H}(\mathbf{k})$. The counting function of this restriction is denoted by $N(\lambda, H(\mathbf{k}) ; \mathcal{C})$, and the density of states by $D(\lambda ; H ; \mathcal{C})$. The same notation can be naturally introduced for any self-adjoint PDO $A$ such that $A(\mathbf{k})\lceil\mathfrak{H}(\mathbf{k})$ has a discrete spectrum. For instance, this is the case for any symbol $a \in \mathbf{S}_{0}(\langle\boldsymbol{\xi}\rangle, \Gamma)$, such that for some bounded $\mathcal{C}$ the subspace $\mathcal{H}(\mathcal{C})$ is invariant for $A$. Note that the condition that $\mathcal{H}(\mathcal{C})$ is invariant for a bounded $\mathcal{C}$ automatically implies that the symbol $h$ is a trigonometric polynomial in $\mathbf{x}$, and that the operator $H(\mathbf{k}) \uparrow \mathfrak{H}(\mathbf{k})$ is finite-dimensional. If $\mathcal{C}$ consists of two disjoint components, i.e., $\mathcal{C}=\mathcal{C}_{1} \cup \mathcal{C}_{2}$ with $\mathcal{C}_{1} \cap \mathcal{C}_{2}=\emptyset$, then obviously, $D(\lambda ; H ; \mathcal{C})=D\left(\lambda ; H ; \mathcal{C}_{1}\right)+D\left(\lambda ; H ; \mathcal{C}_{2}\right)$. Sometimes we indicate which lattice of periodicity is used to compute the partial density of states and write $D_{\Gamma}(\lambda ; H ; \mathcal{C})$. On the other hand, similarly to the "full" density of states (see Theorem 2.2), one can easily show that $D(\lambda ; H ; \mathcal{C})$ does not depend on the choice of the lattice of periodicity for the symbol $h(\mathbf{x}, \boldsymbol{\xi})$.

The following three statements provide three important examples involving the density of states, in which the answer can be computed either completely or 
partially in terms of the symbol. The first statement provides an explicit formula for $D(\lambda)$ in the case of a symbol with constant coefficients. Before proceeding we introduce a convenient notation. Let $a \in \mathrm{L}_{\mathrm{loc}}^{\infty}\left(\mathbb{R}^{d}\right)$ be a real-valued function. Denote

$$
\mathcal{E}(\lambda ; a)=\left\{\boldsymbol{\xi} \in \mathbb{R}^{d}: a(\boldsymbol{\xi}) \leq \lambda\right\}, \forall \lambda \in \mathbb{R} .
$$

Proposition 2.4 Let $A=\mathrm{Op}(a)$ with a real-valued symbol $a \in \mathrm{L}_{\mathrm{loc}}^{\infty}\left(\mathbb{R}^{d}\right)$ such that $a(\boldsymbol{\xi}) \rightarrow \infty$ as $|\boldsymbol{\xi}| \rightarrow \infty$. Let $\mathcal{C} \subset \mathbb{R}^{d}$. Then

$$
D(\lambda ; A ; \mathcal{C})=\frac{1}{(2 \pi)^{d}} \operatorname{vol}(\mathcal{C} \cap \mathcal{E}(\lambda ; a)) .
$$

Proof. Denote

$$
\vartheta(t)=\left\{\begin{array}{l}
0, t<0 \\
1, t \geq 0
\end{array}\right.
$$

Observe that the eigenvalues of $A(\mathbf{k}) \mathcal{P}(\mathbf{k})$ equal $a(\boldsymbol{\mu}+\mathbf{k}), \boldsymbol{\mu}+\mathbf{k} \in \mathcal{C}, \boldsymbol{\mu} \in \Gamma^{\dagger}$, so that

$$
(2 \pi)^{d} D(\lambda)=\sum_{\boldsymbol{\mu}+\mathbf{k} \in{\mathrm{C} \cap \Gamma^{\dagger}}^{\dagger}} \int_{\mathcal{O}^{\dagger}} \vartheta(\lambda-a(\boldsymbol{\mu}+\mathbf{k})) d \mathbf{k}=\int_{a(\boldsymbol{\xi}) \leq \lambda, \boldsymbol{\xi} \in \mathcal{C}} d \boldsymbol{\xi},
$$

as required.

The next lemma deals with the integral of the density of states.

Lemma 2.5 Let $\mathcal{C} \subset \mathbb{R}^{d}$ be a bounded set. Suppose that $h(\mathbf{x}, \boldsymbol{\xi})$ is a symbol of the form (2.8) such that the subspace $\mathcal{H}(\mathcal{C})$ is invariant for $H$. If $\lambda \geq\|\mathcal{P}(\mathcal{C}) H\|$, then

$$
(2 \pi)^{d} \int_{-\infty}^{\lambda} D(\mu ; H ; \mathcal{C}) d \mu=\lambda|\mathcal{C}|-\frac{1}{\mathrm{~d}(\Gamma)} \int_{\mathcal{C}} \int_{\mathcal{O}_{\Gamma}} h(\mathbf{x}, \boldsymbol{\xi}) d \mathbf{x} d \boldsymbol{\xi} .
$$

Proof. For any self-adjoint operator $A$ in a finite-dimensional Hilbert space $\mathfrak{E}$ one can write

$$
\int_{-\infty}^{\lambda} N(\mu ; A) d \mu=\lambda \operatorname{dim} \mathfrak{E}-\operatorname{tr} A, \forall \lambda \geq\|A\| .
$$

Therefore for $\mathfrak{H}(\mathbf{k})=\mathcal{P}(\mathbf{D}+\mathbf{k}, \mathcal{C}) \mathfrak{H}$, we have

$$
\int_{-\infty}^{\lambda} N(\mu ; H(\mathbf{k}) ; \mathcal{C}) d \mu=\lambda \#\left\{\boldsymbol{\gamma}^{\dagger} \in \Gamma^{\dagger}: \boldsymbol{\gamma}^{\dagger}+\mathbf{k} \in \mathcal{C}\right\}-\operatorname{tr}(H(\mathbf{k})\lceil\mathfrak{H}(\mathbf{k})),
$$

if $\lambda \geq\|\mathcal{P}(\mathcal{C}) B\|$. Integrating the first term in $\mathbf{k}$, we obtain $\lambda|\mathcal{C}|$. To find the second term write the matrix elements of the operator $H(\mathbf{k})$ on the subspace $\mathfrak{H}(\mathbf{k})$ :

$$
H_{\boldsymbol{\omega}^{\dagger}, \boldsymbol{\gamma}^{\dagger}}=\frac{1}{\mathrm{~d}(\Gamma)} \int_{\mathcal{O}_{\Gamma}} e^{-i\left\langle\mathbf{x}, \boldsymbol{\omega}^{\dagger}-\boldsymbol{\gamma}^{\dagger}\right\rangle} h\left(\mathbf{x}, \boldsymbol{\gamma}^{\dagger}+\mathbf{k}\right) d \mathbf{x}, \quad \boldsymbol{\omega}^{\dagger}, \boldsymbol{\gamma}^{\dagger} \in \mathcal{C}-\mathbf{k},
$$


so that

$$
\operatorname{tr}\left(H(\mathbf{k})\lceil\mathfrak{H}(\mathbf{k}))=\sum_{\boldsymbol{\gamma}^{\dagger}: \boldsymbol{\gamma}^{\dagger}+\mathbf{k} \in \mathcal{C}} H_{\boldsymbol{\gamma}^{\dagger}, \boldsymbol{\gamma}^{\dagger}}=\sum_{\boldsymbol{\gamma}^{\dagger}: \boldsymbol{\gamma}^{\dagger}+\mathbf{k} \in \mathcal{C}} \frac{1}{\mathrm{~d}(\Gamma)} \int_{\mathcal{O}_{\Gamma}} h\left(\mathbf{x}, \boldsymbol{\gamma}^{\dagger}+\mathbf{k}\right) d \mathbf{x},\right.
$$

After integrating it in $\mathbf{k}$, we obtain the expected integral, thereby completing the proof.

In the next example we consider a PDO which admits a partial separation of variables. To illustrate we use the second order elliptic periodic operators, although the argument below can be easily extended to more general operators. Let $n, l$ be two natural numbers such that $n+l=d$. Let $\mathbf{x}=(\mathbf{y}, \mathbf{t})$ and $\boldsymbol{\xi}=(\boldsymbol{\eta}, \boldsymbol{\omega})$ with $\mathbf{y}, \boldsymbol{\eta} \in \mathbb{R}^{n}$ and $\mathbf{t}, \boldsymbol{\omega} \in \mathbb{R}^{l}$. Let $b=b(\mathbf{y}, \boldsymbol{\eta})$ be a symmetric elliptic symbol of second order on $\mathbb{R}^{n} \times \mathbb{R}^{n}$ and periodic in $\mathbf{y}$ w.r.t. the lattice $\Lambda \subset \mathbb{R}^{n}$. Let $a(\boldsymbol{\omega})=|\mathbf{R} \boldsymbol{\omega}|^{2}$, with a non-degenerate matrix $\mathbf{R}$. Then the operator $H=I \otimes \mathrm{Op}(a)+\mathrm{Op}(b) \otimes I$ is self-adjoint on $\mathrm{H}^{2}\left(\mathbb{R}^{d}\right)$.

Lemma 2.6 Let the operator $H$ be as above and let $\mathcal{C} \subset \mathbb{R}^{l}, \mathcal{D} \subset \mathbb{R}^{n}$ be subsets of $\mathbb{R}^{l}$ and $\mathbb{R}^{n}$. Then

$$
D(\lambda, H ; \mathcal{D} \times \mathcal{C})=\frac{1}{(2 \pi)^{l}} \int_{\mathcal{C}} D(\lambda-a(\mathbf{k}), B ; \mathcal{D}) d \mathbf{k} .
$$

Proof. The symbol of $H$ is periodic w.r.t. the lattice $\Lambda \times(2 \pi \mathbb{Z})^{l}$ with the fundamental domain $\mathcal{M}=\mathcal{O} \times[0,2 \pi)^{l}$. The Floquet representative of the operator $H$ is the operator

$$
H(\mathbf{K})=I \otimes A(\mathbf{k})+B(\boldsymbol{\mu}) \otimes I, \mathbf{K}=(\boldsymbol{\mu}, \mathbf{k}), \mathbf{k} \in[0,1)^{l}, \boldsymbol{\mu} \in \mathcal{O}^{\dagger} .
$$

Since the symbol $a$ does not depend on $\mathbf{x}$, after separating variables we have

$$
N(\lambda, H(\boldsymbol{\mu}, \mathbf{k}) ; \mathcal{D} \times \mathcal{C})=\sum_{\mathbf{m} \in \mathbb{Z}^{l}, \mathbf{m}+\mathbf{k} \in \mathcal{C}} N(\lambda, a(\mathbf{m}+\mathbf{k})+B(\boldsymbol{\mu}) ; \mathcal{D}), \forall \boldsymbol{\mu} \in \mathcal{O}^{\dagger},
$$

and thus

$$
D(\lambda, H ; \mathcal{D} \times \mathcal{C})=\frac{1}{(2 \pi)^{l+n}} \int_{\mathcal{C}} \int_{\mathcal{O}^{\dagger}} N(\lambda, a(\mathbf{k})+B(\boldsymbol{\mu}) ; \mathcal{D}) d \boldsymbol{\mu} d \mathbf{k},
$$

which leads to the required formula.

\section{Properties of periodic PDO's}

In this section we collect various properties of periodic PDO's to be used in what follows. 


\subsection{Some basic results on the calculus of periodic PDO's}

We begin by listing without proofs the results established in [25].

Recall that $\mathbf{S}\left(\mathbb{R}^{d}\right)$ is taken as a natural domain of $\mathrm{Op}(b)$. Unless otherwise stated, all the symbols are supposed to belong to the class $\mathbf{S}_{\alpha}=\mathbf{S}_{\alpha}(w, \Gamma)$ with an arbitrary function $w$ satisfying (2.1) and a lattice $\Gamma$. The function $w$ and the lattice $\Gamma$ are usually omitted from the notation.

Proposition 3.1 Assume that $b \in \mathbf{P}_{0}$. Then $B=\mathrm{Op}(b)$ is bounded in $\mathcal{H}$ and $\|B\| \leq C_{l}|b|_{l, 0}^{(0)}, \forall l>d$, with a constant $C=C_{l}$ independent of $b$.

Remark 3.2 The above proposition automatically implies that PDO's with symbols $b \in \mathbf{S}_{\alpha}(L, \Gamma)$ are bounded for any $\alpha \in \mathbb{R}$ and $\|\mathrm{Op}(b)\| \leq C_{l} L^{\alpha}|b|_{l, 0}^{(\alpha)}$.

Since $\mathrm{Op}(b) u \in \mathbf{S}\left(\mathbb{R}^{d}\right)$ for any $b \in \mathbf{S}_{\alpha}$ and $u \in \mathbf{S}\left(\mathbb{R}^{d}\right)$, the product $\mathrm{Op}(b) \mathrm{Op}(g)$, $b \in \mathbf{S}_{\alpha}, g \in \mathbf{S}_{\beta}$, is well defined on $\mathbf{S}\left(\mathbb{R}^{d}\right)$. A straightforward calculation leads to the following formula for the symbol $b \circ g$ of the product $\mathrm{Op}(b) \mathrm{Op}(g)$ :

$$
(b \circ g)(\mathbf{x}, \boldsymbol{\xi})=\frac{1}{\mathrm{~d}(\Gamma)} \sum_{\boldsymbol{\theta}, \boldsymbol{\phi}} \hat{b}(\boldsymbol{\theta}, \boldsymbol{\xi}+\boldsymbol{\phi}) \hat{g}(\boldsymbol{\phi}, \boldsymbol{\xi}) e^{i(\boldsymbol{\theta}+\boldsymbol{\phi}) \mathbf{x}},
$$

and hence

$$
\widehat{(b \circ g)}(\boldsymbol{\chi}, \boldsymbol{\xi})=\frac{1}{\sqrt{\mathrm{d}(\Gamma)}} \sum_{\boldsymbol{\theta}+\boldsymbol{\phi}=\boldsymbol{\chi}} \hat{b}(\boldsymbol{\theta}, \boldsymbol{\xi}+\boldsymbol{\phi}) \hat{g}(\boldsymbol{\phi}, \boldsymbol{\xi}), \boldsymbol{\chi} \in \Gamma^{\dagger}, \boldsymbol{\xi} \in \mathbb{R}^{d} .
$$

Here and below $\boldsymbol{\theta}, \boldsymbol{\phi} \in \Gamma^{\dagger}$.

Proposition 3.3 Let $b \in \mathbf{S}_{\alpha}, g \in \mathbf{S}_{\beta}$. Then $b \circ g \in \mathbf{S}_{\alpha+\beta}$ and

$$
\left.\left|b \circ g \mathbf{|}_{p, s}^{(\alpha+\beta)} \leq C_{l, s}\right| b\right|_{l, s} ^{(\alpha)} \mid g \mathbf{|}_{l, s}^{(\beta)},
$$

for some $l=l(p)$.

We are also interested in the estimates for symbols of commutators. For PDO's $A, \Psi_{l}, l=1,2, \ldots, N$, denote

$$
\begin{gathered}
\operatorname{ad}\left(A ; \Psi_{1}, \Psi_{2}, \ldots, \Psi_{N}\right)=i\left[\operatorname{ad}\left(A ; \Psi_{1}, \Psi_{2}, \ldots, \Psi_{N-1}\right), \Psi_{N}\right], \\
\operatorname{ad}(A ; \Psi)=i[A, \Psi], \operatorname{ad}^{N}(A ; \Psi)=\operatorname{ad}(A ; \Psi, \Psi, \ldots, \Psi), \operatorname{ad}^{0}(A ; \Psi)=A .
\end{gathered}
$$

For the sake of convenience we use the notation $\operatorname{ad}\left(a ; \psi_{1}, \psi_{2}, \ldots, \psi_{N}\right)$ and $\operatorname{ad}^{N}(a, \psi)$ for the symbols of multiple commutators. It follows from (3.1) that the Fourier coefficients of the symbol ad $(b, g)$ are given by

$$
\begin{array}{r}
\widehat{\operatorname{ad}(b, g)}(\boldsymbol{\chi}, \boldsymbol{\xi})=\frac{i}{\sqrt{\mathrm{d}(\Gamma)}} \sum_{\boldsymbol{\theta}+\boldsymbol{\phi}=\chi}[\hat{b}(\boldsymbol{\theta}, \boldsymbol{\xi}+\boldsymbol{\phi}) \hat{g}(\boldsymbol{\phi}, \boldsymbol{\xi})-\hat{b}(\boldsymbol{\theta}, \boldsymbol{\xi}) \hat{g}(\boldsymbol{\phi}, \boldsymbol{\xi}+\boldsymbol{\theta})], \\
\boldsymbol{\chi} \in \Gamma^{\dagger}, \boldsymbol{\xi} \in \mathbb{R}^{d} .
\end{array}
$$


Proposition 3.4 Let $b \in \mathbf{S}_{\alpha}$ and $g_{j} \in \mathbf{S}_{\beta_{j}}, j=1,2, \ldots, N$. Then $\operatorname{ad}\left(b ; g_{1}, \ldots, g_{N}\right) \in$ $\mathbf{S}_{\gamma}$ with $\gamma=\alpha+\sum_{j=1}^{N}\left(\beta_{j}-1\right)$, and

$$
\mathbf{a d}\left(b ; g_{1}, \ldots, g_{N}\right) \mathbf{|}_{l, s}^{(\gamma)} \leq C_{l, s}|b|_{p, s+N}^{(\alpha)} \prod_{j=1}^{N} \mid g_{j} \mathbf{|}_{p, s+N-j+1}^{\left(\beta_{j}\right)}
$$

with some $p=p\left(l, N, s, \alpha, \beta_{j}\right)$, and a constant $C_{l, s}$ independent of $b, g_{j}$.

\subsection{Asymptotics in the case $d=1$}

An important part is played by the result obtained in [25] for a PDO acting in $\mathrm{L}^{2}(\mathbb{R})$. We state this result in the form convenient for our purposes. Let $b \in$ $\mathbf{S}_{0}(\langle\xi\rangle, \Lambda)$ and $A=\mathrm{Op}\left(a_{0}\right)+\mathrm{Op}(b)$ with $a_{0}(\xi)=g \xi^{2}$ with a constant $g>0$. We assume that $\Lambda$ is a one-dimensional lattice with period $\tau>0$, so that $\mathrm{d}(\Lambda)=\tau$. Note that here and everywhere below, in the case $d=1$ we use the notation $x$ and $\xi$ instead of the boldface letters $\mathbf{x}, \boldsymbol{\xi}$.

Proposition 3.5 Let $d=1$. Suppose that $b \in \mathbf{S}_{0}(\langle\xi\rangle, \Lambda)$ is a $\tau$-periodic symmetric symbol. Then the density of states $D(\lambda ; A)$ is given by the formula

$$
\begin{gathered}
2 \pi D(\lambda ; A)=2 \sqrt{\frac{|\lambda|}{g}}-\frac{1}{\sqrt{\langle\lambda\rangle g}} \mathcal{A}(\lambda, g)+O\left(\langle\lambda\rangle^{-3 / 2}\right), \forall \lambda \in \mathbb{R}, \\
\mathcal{A}(\lambda, g)=\frac{1}{2 \tau} \int_{0}^{\tau}\left(b\left(x, \sqrt{|\lambda| g^{-1}}\right)+b\left(x,-\sqrt{|\lambda| g^{-1}}\right)\right) d x .
\end{gathered}
$$

This formula is uniform in the symbol $b$ and in the parameters $g$ and $\tau$ satisfying the bounds $c \leq g \leq C, c \leq \tau \leq C$.

Remark that the above formula has an asymptotic meaning only for large $\lambda$, and for bounded $\lambda$ we can only claim that the density $D(\lambda ; A)$ is bounded from above uniformly in $b$. However it is useful to express these two facts in one formula which is valid for all $\lambda \in \mathbb{R}$.

In the study of the density of states for the multidimensional case we shall encounter integrals involving densities for lower-dimensional operators. In particular, there is a need to calculate integrals of the density of states for the one-dimensional case, of the type

$$
D_{p}(\mu, \lambda ; A)=\int_{-\infty}^{\mu} D(t ; A)(\lambda-t)^{\frac{p}{2}} d t, \mu<\lambda, p \in \mathbb{R} .
$$

In the next lemma we apply Lemma 2.5 and Proposition 3.5 to compare the above integral for the operator $A=A_{0}+\mathrm{Op}(b)$ with that for the "unperturbed" operator $A^{o}=A_{0}+\mathrm{Op}\left(b^{o}\right)$, where $b^{o}=b^{o}(\xi)$ is the mean value of $b(x, \xi)$ :

$$
b^{o}(\xi)=\frac{1}{\tau} \int_{0}^{\tau} b(x, \xi) d x
$$


Lemma 3.6 Let $\ell=\left[-L g^{-1 / 2}, L g^{-1 / 2}\right], L>0$. Suppose that $b \in \mathbf{S}_{0}(\langle\xi\rangle, \tau \mathbb{Z})$ is a $\tau$-periodic symmetric symbol such that

$$
\left\|\mathrm{Op}\left(b^{o}\right)\right\| \leq L^{2},\left\|\mathrm{Op}\left(b-b^{o}\right)\right\| \leq L^{2} .
$$

Suppose also that the subspace $\mathcal{H}(\ell)$ is reducing for the operator $\mathrm{Op}(b)$ and that $\mathcal{P}(\mathbb{R} \backslash \ell) \mathrm{Op}(b)=\mathrm{Op}\left(b^{\circ}\right)$. Then for $\lambda / 2 \geq \mu \geq 3 L^{2}$ and $p \in \mathbb{R}$ one has

$$
\left|D_{p}(\mu, \lambda ; A)-D_{p}\left(\mu, \lambda ; A^{o}\right)\right| \leq C \lambda^{\frac{p-2}{2}} L .
$$

The constant $C=C(p)$ in (3.7) is finite for all $p \in \mathbb{R}$. In particular,

$$
D_{0}(\mu, \lambda ; A)=D_{0}\left(\mu, \lambda ; A^{o}\right) .
$$

The constant $C(p)$ is independent of $\mu, \lambda$ and uniform in the symbol $b$ and in the parameters $g$ and $\tau$ satisfying the conditions $c \leq \tau \leq C, c \leq g \leq C$.

Proof. Compare the densities for $A$ and $A^{o}$ :

$$
\begin{aligned}
D_{p}(\mu, \lambda ; A)= & D_{p}\left(\mu, \lambda ; A^{o}\right) \\
+ & \int_{-\infty}^{\mu}\left(D(t ; A)-D\left(t ; A^{o}\right)\right)\left[(\lambda-t)^{\frac{p}{2}}-\lambda^{\frac{p}{2}}\right] d t \\
& +\lambda^{\frac{p}{2}} \int_{-\infty}^{\mu}\left(D(t ; A)-D\left(t ; A^{o}\right)\right) d t .
\end{aligned}
$$

Let us consider the second integral first. Since $\mathcal{P}(\mathbb{R} \backslash \ell) \mathrm{Op}(b)=\mathrm{Op}\left(b^{o}\right)$, we have

$$
D(t ; A)=D(t ; A ; \ell)+D\left(t ; A^{o} ; \mathbb{R} \backslash \ell\right),
$$

and hence

$$
D(t ; A)-D\left(t ; A^{o}\right)=D(t ; A ; \ell)-D\left(t ; A^{o} ; \ell\right) .
$$

To find the integrals of the terms in the r.h.s. use Lemma 2.5. In view of (3.6), $\|A \mathcal{P}(\ell)\| \leq L^{2}+\|\mathrm{Op}(b)\| \leq 3 L^{2}$. By the conditions of the lemma this does not exceed $\mu$, and therefore, by Lemma 2.5 and (3.5),

$$
\begin{aligned}
2 \pi \int_{-\infty}^{\mu} D(t ; A ; \ell) d t & =2 \mu L g^{-1 / 2}-\frac{1}{\tau} \int_{\ell} \int_{0}^{\tau}\left(g \xi^{2}+b(x, \xi)\right) d x d \xi \\
& =2 \pi \int_{-\infty}^{\mu} D\left(t ; A^{o} ; \ell\right) d t .
\end{aligned}
$$

Hence the second integral in (3.8) vanishes.

To handle the first integral in (3.8) we note that in view of Proposition 3.5

$$
\left|D(t ; A)-D\left(t ; A^{o}\right)\right| \leq C|t|^{-3 / 2},
$$


for all $t \neq 0$ with a constant $C$ uniform in the symbol $b$. Notice also that in view of the estimates (3.6) and the property that $\mathcal{P}(\mathbb{R} \backslash \ell) \mathrm{Op}(b)=\mathrm{Op}\left(b^{\circ}\right)$, we have $D(t ; A)=D\left(t ; A^{o}\right)$ for all $t \geq 3 L^{2}$, and thus the limits of integration can be replaced by $-3 L^{2}$ and $3 L^{2}$. Now, using the straightforward estimate

$$
\left|(\lambda-t)^{\frac{p}{2}}-\lambda^{\frac{p}{2}}\right| \leq C \lambda^{\frac{p}{2}-1}|t|,|t| \leq \mu \leq \lambda / 2,
$$

with a constant $C=C(p)$, we conclude that

$$
\begin{aligned}
\mid \int_{-3 L^{2}}^{3 L^{2}}\left(D(t ; A)-D\left(t ; A^{o}\right)\right) & {\left[(\lambda-t)^{\frac{p}{2}}-\lambda^{\frac{p}{2}}\right] d t \mid } \\
\leq & C \lambda^{\frac{p-2}{2}} \int_{-3 L^{2}}^{3 L^{2}}|t|^{-1 / 2} d t \\
\leq & \tilde{C} \lambda^{\frac{p-2}{2}} L .
\end{aligned}
$$

The last inequality and the formula (3.8) lead to (3.7).

\subsection{Linear change of variables}

It is an elementary exercise to describe how the symbols of PDO's and their densities of state transform under a linear change of variables. Since in what follows we heavily use various changes of variables, below we state these elementary formulae in the form of Lemmas.

Let $a \in \mathbf{P}_{\alpha}(w, \Gamma)$ with a function $w$ satisfying (2.1). Let $\mathbf{M}: \mathbb{R}^{d} \rightarrow \mathbb{R}^{d}$ be a non-degenerate linear map. Note first of all that the lattice $\Gamma$ transforms into another lattice which we denote $\tilde{\Gamma}=\mathbf{M} \Gamma$. Also, $\tilde{\Gamma}^{\dagger}=\left(\mathbf{M}^{T}\right)^{-1} \Gamma^{\dagger}$. It is straightforward to see that the sets

$$
\mathcal{O}_{\tilde{\Gamma}}=\mathbf{M} \mathcal{O}_{\Gamma}, \quad \mathcal{O}_{\tilde{\Gamma}}^{\dagger}=\left(\mathbf{M}^{T}\right)^{-1} \mathcal{O}_{\Gamma}^{\dagger}
$$

are fundamental sets of the lattices $\tilde{\Gamma}$ and $\tilde{\Gamma}^{\dagger}$ respectively, and that

$$
\mathrm{d}(\tilde{\Gamma})=\operatorname{det} \mathbf{M} \mathrm{d}(\Gamma) .
$$

Define the unitary operator $W=W_{\mathbf{M}}: \mathcal{H} \rightarrow \mathcal{H}$ for any $u \in \mathcal{H}$ as follows:

$$
(W u)(\mathbf{t})=\sqrt{\operatorname{det} \mathbf{M}} u(\mathbf{M t}) .
$$

Lemma 3.7 Let $\mathbf{M}$ be a non-degenerate linear map from $\mathbb{R}^{d}$ to $\mathbb{R}^{d}$, and let $a \in$ $\mathbf{P}_{\alpha}(w, \Gamma)$. Then the symbol $b(\mathbf{x}, \boldsymbol{\xi})$ of the operator $B=W^{*} \mathrm{Op}(a) W$ is given by

$$
b(\mathbf{x}, \boldsymbol{\xi})=a\left(\mathbf{M}^{-1} \mathbf{x}, \mathbf{M}^{T} \boldsymbol{\xi}\right) .
$$

This symbol is $\tilde{\Gamma}$-periodic with $\tilde{\Gamma}=\mathbf{M} \Gamma$, the Fourier transform $\hat{b}(\boldsymbol{\theta}, \boldsymbol{\xi})$ of the symbol $b$ is given by

$$
\hat{b}(\boldsymbol{\theta}, \boldsymbol{\xi})=\sqrt{\operatorname{det} \mathbf{M}} \hat{a}\left(\mathbf{M}^{T} \boldsymbol{\theta}, \mathbf{M}^{T} \boldsymbol{\xi}\right), \boldsymbol{\theta} \in \tilde{\Gamma}^{\dagger},
$$

and $b \in \mathbf{P}_{\alpha}(\tilde{w}, \tilde{\Gamma}), \tilde{w}(\boldsymbol{\xi})=w\left(\mathbf{M}^{T} \boldsymbol{\xi}\right)$. Moreover, if $a \in \mathbf{S}_{\alpha}(w, \Gamma)$, then $b \in \mathbf{S}_{\alpha}(\tilde{w}, \tilde{\Gamma})$. 
Proof. The formula (3.10) for $b$ follows by a direct elementary calculation. By inspection the symbol $b$ is periodic with respect to the lattice $\tilde{\Gamma}$. To prove (3.11) write, using (3.10):

$$
\begin{aligned}
\hat{b}(\boldsymbol{\theta}, \boldsymbol{\xi}) & =\frac{1}{\sqrt{\mathrm{d}(\tilde{\Gamma})}} \int_{\mathcal{O}_{\tilde{\Gamma}}} a\left(\mathbf{M}^{-1} \mathbf{x}, \mathbf{M}^{T} \boldsymbol{\xi}\right) e^{-i\langle\boldsymbol{\theta}, \mathbf{x}\rangle} d \mathbf{x} \\
& =\frac{\operatorname{det} \mathbf{M}}{\sqrt{\mathrm{d}(\tilde{\Gamma})}} \int_{\mathcal{O}_{\Gamma}} a\left(\mathbf{x}, \mathbf{M}^{T} \boldsymbol{\xi}\right) e^{-i\left\langle\mathbf{M}^{T} \boldsymbol{\theta}, \mathbf{x}\right\rangle} d \mathbf{x}=\sqrt{\operatorname{det} \mathbf{M}} \hat{a}\left(\mathbf{M}^{T} \boldsymbol{\theta}, \mathbf{M}^{T} \boldsymbol{\xi}\right) .
\end{aligned}
$$

This proves (3.11).

Now it is immediate to see that $b \in \mathbf{P}_{\alpha}(\tilde{w}, \tilde{\Gamma})$ if $a \in \mathbf{P}_{\alpha}(w, \Gamma)$, or, $b \in \mathbf{S}_{\alpha}(\tilde{w}, \tilde{\Gamma})$ if $a \in \mathbf{S}_{\alpha}(w, \Gamma)$.

Let us find out how the density of states changes under the change of variables.

Lemma 3.8 Let $\mathbf{M}$ be a non-degenerate linear map from $\mathbb{R}^{d}$ to $\mathbb{R}^{d}$, and let a be $a \Gamma$-periodic elliptic symbol such that $\mathcal{H}(\mathcal{C}), \mathcal{C} \subset \mathbb{R}^{d}$ is an invariant subspace for $A=\mathrm{Op}(a)$. Then the subspace $\mathcal{H}\left(\left(\mathbf{M}^{T}\right)^{-1} \mathrm{C}\right)$ is invariant for $B=W^{*} A W, W=$ $W_{\mathbf{M}}$ and

$$
D_{\Gamma}(\lambda ; A ; \mathcal{C})=\operatorname{det} \mathbf{M} D_{\tilde{\Gamma}}\left(\lambda ; W^{*} A W ;\left(\mathbf{M}^{T}\right)^{-1} \mathcal{C}\right) .
$$

In particular, if $\mathcal{C}=\mathbb{R}^{d}$, then

$$
D_{\Gamma}(\lambda ; A)=\operatorname{det} \mathbf{M} D_{\tilde{\Gamma}}\left(\lambda ; W^{*} A W\right) .
$$

Proof. If $\mathcal{H}(\mathcal{C})$ is invariant for $A$, then the invariance of $\mathcal{H}\left(\mathrm{C}^{\prime}\right), \mathrm{C}^{\prime}=\left(\mathbf{M}^{T}\right)^{-1} \mathcal{C}$, for $B$ follows from the formula $\mathcal{P}\left(\mathfrak{C}^{\prime}\right)=W^{*} \mathcal{P}(\mathcal{C}) W$.

The representatives in the Floquet decomposition of $A$ and $B=W^{*} A W$ are the operators $A(\mathbf{k}), \mathbf{k} \in \mathcal{O}_{\Gamma}^{\dagger}$ and $B(\tilde{\mathbf{k}}), \tilde{\mathbf{k}} \in \mathcal{O}_{\tilde{\Gamma}}^{\dagger}$ acting in $\mathrm{L}^{2}\left(\mathcal{O}_{\Gamma}\right)$ and $\mathrm{L}^{2}\left(\mathcal{O}_{\tilde{\Gamma}}\right)$ respectively, with the symbols

$$
\begin{gathered}
a\left(\mathbf{x}, \boldsymbol{\gamma}^{\dagger}+\mathbf{k}\right), \boldsymbol{\gamma}^{\dagger} \in \Gamma^{\dagger}, \text { and } \\
b\left(\mathbf{x}, \tilde{\gamma}^{\dagger}+\tilde{\mathbf{k}}\right)=a\left(\mathbf{M}^{-1} \mathbf{x}, \boldsymbol{\gamma}^{\dagger}+\mathbf{M}^{T} \tilde{\mathbf{k}}\right), \tilde{\boldsymbol{\gamma}}^{\dagger}=\left(\mathbf{M}^{T}\right)^{-1} \boldsymbol{\gamma}^{\dagger} \in \tilde{\Gamma}^{\dagger}
\end{gathered}
$$

As in Lemma 3.7 one checks directly that $B(\tilde{\mathbf{k}})=W^{*} A\left(\mathbf{M}^{T} \tilde{\mathbf{k}}\right) W$, and therefore

$$
\begin{aligned}
(2 \pi)^{d} D_{\tilde{\Gamma}}\left(\lambda ; B ; \mathcal{C}^{\prime}\right) & =\int_{\mathcal{O}_{\tilde{\Gamma}}^{\dagger}} N\left(\lambda, B(\tilde{\mathbf{k}}) ; \mathcal{C}^{\prime}\right) d \tilde{\mathbf{k}}=\int_{\mathcal{O}_{\Gamma}^{\dagger}} N\left(\lambda, A\left(\mathbf{M}^{T} \tilde{\mathbf{k}}\right) ; \mathcal{C}\right) d \tilde{\mathbf{k}} \\
& =\frac{1}{\operatorname{det} \mathbf{M}} \int_{\mathcal{O}_{\Gamma}^{\dagger}} N(\lambda, A(\mathbf{k}) ; \mathcal{C}) d \mathbf{k}=\frac{(2 \pi)^{d}}{\operatorname{det} \mathbf{M}} D_{\Gamma}(\lambda ; A ; \mathcal{C}) .
\end{aligned}
$$




\subsection{A special orthogonal transformation}

A special role in what follows will be played by an orthogonal change of variables associated with a vector $\boldsymbol{\nu} \in \mathbb{R}^{d}$. From now on we use the notation

$$
\mathbf{n}(\boldsymbol{\nu})=\boldsymbol{\nu}|\boldsymbol{\nu}|^{-1}, \quad \mathbf{0} \neq \boldsymbol{\nu} \in \mathbb{R}^{d} .
$$

Recall that $\mathbf{e}_{j}, j=1,2, \ldots, d$ denote the vectors of the standard orthonormal basis in $\mathbb{R}^{d}$. Let $\mathbf{M}=\mathbf{M}(\boldsymbol{\nu})$ be an orthogonal transformation $\mathbf{M}: \mathbb{R}^{d} \rightarrow \mathbb{R}^{d}$ such that $\mathbf{e}_{1}=\mathbf{M n}(\boldsymbol{\nu})$. Clearly, $\mathbf{M}(t \boldsymbol{\nu})=\mathbf{M}(\boldsymbol{\nu})$ for any real $t>0$. Let us find out how this transformation acts on certain domains in $\mathbb{R}^{d}$. Let $L \geq 0, s \geq 0$ be some numbers. Now define the domains

$$
\begin{aligned}
\Lambda_{\boldsymbol{\nu}} & =\left\{\boldsymbol{\xi} \in \mathbb{R}^{d}:|\langle\boldsymbol{\xi}, \boldsymbol{\nu}\rangle| \leq L|\boldsymbol{\nu}|\right\} \\
& =\left\{\boldsymbol{\xi} \in \mathbb{R}^{d}:|\langle\boldsymbol{\xi}, \mathbf{n}(\boldsymbol{\nu})\rangle| \leq L\right\} . \\
\Omega_{\boldsymbol{\nu}}(s) & =\left\{\boldsymbol{\xi} \in \mathbb{R}^{d}:|\boldsymbol{\xi}|^{2}-|\langle\boldsymbol{\xi}, \mathbf{n}(\boldsymbol{\nu})\rangle|^{2} \geq s^{2}\right\} .
\end{aligned}
$$

The number $L$ will be kept the same throughout the paper and thus it is not reflected in the notation. The geometrical meaning of the sets $\Lambda_{\nu}$ and $\Omega_{\nu}$ is simple: in particular, $\Omega_{\nu}$ is the set of all vectors $\boldsymbol{\xi}$ such that the distance from $\boldsymbol{\xi}$ to the one-dimensional subspace spanned by $\boldsymbol{\nu}$ is greater than $s$. Clearly, for any $t \neq 0$ one has $\Lambda_{t \nu}=\Lambda_{\nu}$ and $\Omega_{t \nu}=\Omega_{\nu}$.

For the case $d=2$ the definition of $\Omega_{\nu}$ can be simplified. Namely, let

$$
\mathbf{J}=\left[\begin{array}{cc}
0 & 1 \\
-1 & 0
\end{array}\right]
$$

Then for any $\mathbf{t} \in \mathbb{R}^{2}$ the vector $\mathbf{t}^{\perp}=\mathbf{J t}$ is orthogonal to $\mathbf{t}$. Now $\Omega_{\boldsymbol{\nu}}$ can be rewritten as follows:

$$
\Omega_{\boldsymbol{\nu}}(s)=\left\{\boldsymbol{\xi} \in \mathbb{R}^{2}:\left|\left\langle\mathbf{n}^{\perp}(\boldsymbol{\nu}), \boldsymbol{\xi}\right\rangle\right| \geq s\right\} .
$$

The next lemma describes how the sets $\Lambda_{\nu}$ and $\Omega_{\boldsymbol{\nu}}(s)$ transform under $\mathbf{M}(\boldsymbol{\nu})$ :

Lemma 3.9 Let $\mathbf{M}=\mathbf{M}(\boldsymbol{\nu})$ be the orthogonal transformation defined above. Then

$$
\begin{aligned}
\mathbf{M} \Lambda_{\nu} & =\Lambda_{\mathbf{e}_{1}}=\left\{\xi_{1} \in \mathbb{R}:\left|\xi_{1}\right| \leq L\right\} \times \mathbb{R}^{d-1}, \\
\mathbf{M} \Omega_{\boldsymbol{\nu}}(s) & =\Omega_{\mathbf{e}_{1}}(s)=\mathbb{R} \times\left\{\hat{\boldsymbol{\xi}} \in \mathbb{R}^{d-1}:|\hat{\boldsymbol{\xi}}| \geq s\right\} .
\end{aligned}
$$

Proof. As $\mathbf{M} \mathcal{C}=\left\{\boldsymbol{\xi} \in \mathbb{R}^{d}: \mathbf{M}^{T} \boldsymbol{\xi} \in \mathcal{C}\right\}$ for any set $\mathcal{C} \subset \mathbb{R}^{d}$, we have

$$
\begin{aligned}
\mathbf{M} \Lambda_{\nu} & =\left\{\boldsymbol{\xi} \in \mathbb{R}^{d}:\left|\left\langle\mathbf{M}^{T} \boldsymbol{\xi}, \mathbf{n}(\boldsymbol{\nu})\right\rangle\right| \leq L\right\} \\
& =\left\{\boldsymbol{\xi} \in \mathbb{R}^{d}:\left|\left\langle\mathbf{M}^{T} \boldsymbol{\xi}, \mathbf{M}^{T} \mathbf{e}_{1}\right\rangle\right| \leq L\right\}=\Lambda_{\mathbf{e}_{1}} .
\end{aligned}
$$

Here we have used the property $\mathbf{e}_{1}=\mathbf{M n}(\boldsymbol{\nu})$. Similarly for $\Omega_{\boldsymbol{\nu}}(s)$. 


\section{Further properties of periodic PDO's. Partition of symbols}

In this section we describe two procedures of partitioning the symbols, that will play a crucial role in the study of spectral properties of PDO's. Roughly speaking, the aim of these partitions is to transform the operators to a form when separation of variables becomes possible.

\subsection{Partition I}

The first partition is designed to split the symbol into components supported on different parts of the dual space. The general definitions below will be given for symbols $b \in \mathbf{P}_{\alpha}(w, \Gamma)$ with an arbitrary weight $w$, but later we shall make more restrictive assumptions.

Let $\Upsilon \in \mathrm{C}_{0}^{\infty}(\mathbb{R})$ be a non-negative function such that

$$
0 \leq \Upsilon \leq 1, \quad \Upsilon(t)=\left\{\begin{array}{l}
1,|t| \leq 1 / 4 \\
0,|t| \geq 1 / 2
\end{array}\right.
$$

Assume also for convenience that $\Upsilon$ is even, i.e., $\Upsilon(t)=\Upsilon(-t)$. For a number $L \geq 1$ define

$$
\begin{cases}\zeta_{\boldsymbol{\theta}}(\boldsymbol{\xi} ; L)= & \Upsilon\left(\frac{\langle\boldsymbol{\theta}, \boldsymbol{\xi}+\boldsymbol{\theta} / 2\rangle}{|\boldsymbol{\theta}| L}\right), \\ \varphi_{\boldsymbol{\theta}}(\boldsymbol{\xi} ; L)= & 1-\zeta_{\boldsymbol{\theta}}(\boldsymbol{\xi} ; L) .\end{cases}
$$

We point out that

$$
\varphi_{\boldsymbol{\theta}}(\boldsymbol{\xi} ; L)=\varphi_{-\boldsymbol{\theta}}(\boldsymbol{\xi}+\boldsymbol{\theta} ; L), \quad \zeta_{\boldsymbol{\theta}}(\boldsymbol{\xi} ; L)=\zeta_{-\boldsymbol{\theta}}(\boldsymbol{\xi}+\boldsymbol{\theta} ; L),
$$

since the function $\Upsilon$ is even. Note that

$$
\left|\mathbf{D}^{\mathbf{s}} \varphi_{\boldsymbol{\theta}}(\boldsymbol{\xi} ; L)\right|+\left|\mathbf{D}^{\mathbf{s}} \zeta_{\boldsymbol{\theta}}(\boldsymbol{\xi} ; L)\right| \leq C_{s} L^{-s}, s=|\mathbf{s}| .
$$

This inequality shows that the functions $\zeta_{\boldsymbol{\theta}}$ and $\varphi_{\boldsymbol{\theta}}$, viewed as symbols, belong to the class $\mathbf{S}_{0}(L)$.

Using $\varphi_{\boldsymbol{\theta}}, \zeta_{\boldsymbol{\theta}}$, we shall introduce the following linear operations on PDO's. Fix a positive parameter $r$ and let

$$
\begin{array}{r}
\Theta_{r}=\Theta_{r}(\Gamma)=\left\{\boldsymbol{\theta} \in \Gamma^{\dagger}: 0<|\boldsymbol{\theta}| \leq r\right\}, \Theta_{r}^{0}=\Theta_{r} \cup\{\mathbf{0}\}, \\
\Xi_{r}=\Xi_{r}(\Gamma)=\left\{\boldsymbol{\theta} \in \Gamma^{\dagger}:|\boldsymbol{\theta}|>r\right\}=\Gamma^{\dagger} \backslash \Theta_{r}^{0}(\Gamma) .
\end{array}
$$


Given a symbol $b \in \mathbf{S}_{\alpha}(w)$, define four new symbols $b^{o}, b^{\sharp}, b^{b}, b^{\uparrow}$ as follows:

$$
\begin{gathered}
b^{\uparrow}(\mathbf{x}, \boldsymbol{\xi})=\frac{1}{\sqrt{\mathrm{d}(\Gamma)}} \sum_{\boldsymbol{\theta} \in \Xi_{r}} \hat{b}(\boldsymbol{\theta}, \boldsymbol{\xi}) e^{i \boldsymbol{\theta} \mathbf{x}}, \\
b^{\sharp}(\mathbf{x}, \boldsymbol{\xi})=\frac{1}{\sqrt{\mathrm{d}(\Gamma)}} \sum_{\boldsymbol{\theta} \in \Theta_{r}} \hat{b}(\boldsymbol{\theta}, \boldsymbol{\xi}) \varphi_{\boldsymbol{\theta}}(\boldsymbol{\xi} ; L) e^{i \boldsymbol{\theta} \mathbf{x}}, \\
b^{b}(\mathbf{x}, \boldsymbol{\xi})=\frac{1}{\sqrt{\mathrm{d}(\Gamma)}} \sum_{\boldsymbol{\theta} \in \Theta_{r}} \hat{b}(\boldsymbol{\theta}, \boldsymbol{\xi}) \zeta_{\boldsymbol{\theta}}(\boldsymbol{\xi} ; L) e^{i \boldsymbol{\theta} \mathbf{x}}, \\
b^{o}(\mathbf{x}, \boldsymbol{\xi})=b^{o}(\boldsymbol{\xi})=\frac{1}{\sqrt{\mathrm{d}(\Gamma)}} \hat{b}(\mathbf{0}, \boldsymbol{\xi}) .
\end{gathered}
$$

By definition of $\Upsilon$

$$
b=b^{\uparrow}+b^{o}+b^{b}+b^{\sharp} .
$$

The symbol $b^{\uparrow}$ contains only the Fourier coefficients $\hat{b}(\boldsymbol{\theta}, \boldsymbol{\xi})$ with large $\boldsymbol{\theta}$ 's, and later it will be shown not to contribute to the answer. The remaining symbols are trigonometric polynomials in $\mathbf{x}$. The Fourier coefficient $\hat{b}^{b}(\boldsymbol{\theta}, \cdot)$ is supported near the hyperplane $\langle\boldsymbol{\theta}, \boldsymbol{\xi}+\boldsymbol{\theta} / 2\rangle=0$, whereas $\hat{b}^{\sharp}(\boldsymbol{\theta}, \cdot)$ lives away from this hyperplane. It is easy to see that for any symbol $b \in \mathbf{P}_{\alpha}$ the introduced symbols also belong to the same class. The corresponding operators are denoted by

$$
B^{\uparrow}=\mathrm{Op}\left(b^{\uparrow}\right), B^{\sharp}=\mathrm{Op}\left(b^{\sharp}\right), B^{b}=\mathrm{Op}\left(b^{b}\right), B^{o}=\mathrm{Op}\left(b^{o}\right) .
$$

We also denote $B^{b, \uparrow}=B^{b}+B^{\uparrow}$.

Assume now that $b \in \mathbf{S}_{\alpha}(w, \Gamma)$ with a constant weight $w=L^{\prime} \in[1, L]$. Then the operations introduced above preserve the properties of the symbol $b$, that is for any $b \in \mathbf{S}_{\alpha}\left(L^{\prime}, \Gamma\right)$ the symbols $b^{b}, b^{\sharp}, b^{\uparrow}, b^{o}$ belong to the same class and for all $l, s$ and $p<l-d$

$$
\left\{\begin{array}{l}
\left|b^{b}\right|_{l, s}^{(\alpha)}+\left|b^{\sharp}\right|_{l, s}^{(\alpha)}+\left|b^{o}\right|_{l, s}^{(\alpha)} \leq C|b|_{l, s}^{(\alpha)}, \\
\left|b^{\uparrow}\right|_{p, s}^{(\alpha)} \leq C r^{p-l+d}|b|_{l, s}^{(\alpha)} .
\end{array}\right.
$$

The constant $C$ in the above estimates depends on $l, s$ and on the lattice $\Gamma$. The first estimate immediately follows from (4.4). The second bound is a consequence of (2.3) and (4.5). In the case $d=1$ the operation "b" possesses one more useful property:

Lemma 4.1 Let $d=1$ and let $b \in \mathbf{S}_{\alpha}(L, \Gamma), \alpha \leq 0$. Then for $r \leq L$ the symbol $b^{b}$ belongs to $\mathbf{S}_{\alpha}(\langle\xi\rangle, \Gamma)$ and

$$
\left|b^{b}\right|_{l, s ;\langle\xi\rangle}^{(\alpha)} \leq C_{l, s}|b|_{l, s ; L}^{(\alpha)}
$$

with a constant $C_{l, s}$ depending only on $l, s$. 
Proof. The proof is similar to the above proof of (4.10) for the classes with a constant weight. One uses the fact that $|\xi| \leq L / 2+r / 2 \leq L$ on the support of $\zeta_{\theta}$, and thus

$$
\left|\partial_{\xi}^{s} \hat{b}(\theta, \xi)\right| \leq|b|_{l, s ; L}\langle\theta\rangle^{-l} L^{\alpha-s} \leq C_{l, s}|b|_{l, s ; L}\langle\theta\rangle^{-l}\langle\xi\rangle^{\alpha-s}
$$

for all $\xi \in \operatorname{supp} \zeta_{\theta}$ and $\theta \in \Theta_{r}$. It also follows from (4.4) that

$$
\left|\partial_{\xi}^{s} \varphi_{\theta}(\xi ; L)\right|+\left|\partial_{\xi}^{s} \zeta_{\theta}(\xi ; L)\right| \leq C_{s}\langle\xi\rangle^{-s}
$$

The above estimates lead to the proclaimed estimate for the norm $|b|_{l, s ;\langle\xi\rangle}^{(\alpha)}$.

To check that the introduced operations preserve symmetry, calculate using (4.3) and remembering that $\boldsymbol{\theta}$ and $\boldsymbol{- \theta}$ do ( or do not ) belong to $\Theta_{r}$ simultaneously:

$$
\begin{aligned}
\overline{\hat{b}^{b}(-\boldsymbol{\theta}, \boldsymbol{\xi}+\boldsymbol{\theta})} & =\overline{\hat{b}(-\boldsymbol{\theta}, \boldsymbol{\xi}+\boldsymbol{\theta})} \zeta_{-\boldsymbol{\theta}}(\boldsymbol{\xi}+\boldsymbol{\theta} ; L) \\
& =\hat{b}(\boldsymbol{\theta}, \boldsymbol{\xi}) \zeta_{\boldsymbol{\theta}}(\boldsymbol{\xi} ; L)=\hat{b}^{b}(\boldsymbol{\theta}, \boldsymbol{\xi}) .
\end{aligned}
$$

Thus, by (2.6) the operator $B^{b}$ is symmetric if so is $B$. Similarly for $B^{\sharp}$ and $B^{\uparrow}$.

Let us find out how these symbols transform under an orthogonal change of variables $\mathbf{M}$. Below we use the notation for the transformed objects, introduced in the beginning of Subsect. 3.3. Note first a direct consequence of the Definition $(4.2)$

$$
\left\{\begin{array}{l}
\zeta_{\boldsymbol{\theta}}\left(\mathbf{M}^{T} \boldsymbol{\xi} ; L\right)=\zeta_{\boldsymbol{\omega}}(\boldsymbol{\xi} ; L), \\
\varphi_{\boldsymbol{\theta}}\left(\mathbf{M}^{T} \boldsymbol{\xi} ; L\right)=\varphi_{\boldsymbol{\omega}}(\boldsymbol{\xi} ; L),
\end{array} \quad \boldsymbol{\omega}=\mathbf{M} \boldsymbol{\theta} \in \tilde{\Gamma}^{\dagger}=\mathbf{M} \Gamma^{\dagger} .\right.
$$

Lemma 4.2 Let $\mathbf{M}: \mathbb{R}^{d} \rightarrow \mathbb{R}^{d}$ be an orthogonal transformation. Let $a \in \mathbf{P}_{\alpha}(w)$ with some $\alpha \in \mathbb{R}$. Denote by $\natural$ any of the symbols $\uparrow, b$, o or $\sharp$. Then

$$
\left(W_{\mathbf{M}}^{*} A W_{\mathbf{M}}\right)^{\natural}=W_{\mathbf{M}}^{*} A^{\natural} W_{\mathbf{M}} \text {. }
$$

Proof. Let $B=W_{\mathbf{M}}^{*} A W_{\mathbf{M}}$. We consider only the case $\downarrow=b$. By Definition (4.8) the symbol of the operator $B^{b}$ coincides with

$$
\frac{1}{\sqrt{\mathrm{d}(\tilde{\Gamma})}} \sum_{\boldsymbol{\omega} \in \Theta_{r}(\tilde{\Gamma})} \hat{b}(\boldsymbol{\omega}, \boldsymbol{\xi}) \zeta_{\boldsymbol{\omega}}(\boldsymbol{\xi} ; L) e^{i\langle\boldsymbol{\omega}, \mathbf{x}\rangle} .
$$

On the other hand, $\mathbf{M}^{T} \Theta_{r}(\tilde{\Gamma})=\Theta_{r}(\Gamma)$ and by (3.11) the Fourier transform of the symbol of the operator $W_{\mathbf{M}}^{*} A^{\natural} W_{\mathbf{M}}$ is given by

$$
\hat{a}\left(\mathbf{M}^{T} \boldsymbol{\omega}, \mathbf{M}^{T} \boldsymbol{\xi}\right) \zeta_{\mathbf{M}^{T} \boldsymbol{\omega}}\left(\mathbf{M}^{T} \boldsymbol{\xi} ; L\right), \boldsymbol{\omega} \in \tilde{\Gamma}^{\dagger}
$$

In view of $(3.11)$ and (4.11) this coincides with $\hat{b}(\boldsymbol{\omega}, \boldsymbol{\xi}) \zeta_{\boldsymbol{\omega}}(\boldsymbol{\xi} ; L)$, as required. 


\subsection{Partition II}

Here we describe a way to split symbols into components associated with the socalled primitive vectors $\boldsymbol{\nu} \in \Gamma^{\dagger}$. For a symbol $b \in \mathbf{S}_{\alpha}(w, \Gamma)$ introduce a family of associated symbols constructed as follows. For a vector $\mathbf{0} \neq \boldsymbol{\nu} \in \Gamma^{\dagger}$ define the subset

$$
\Gamma_{\nu}^{\dagger}=\{n \boldsymbol{\nu}, 0 \neq n \in \mathbb{Z}\}
$$

Let us introduce the symbol

$$
b_{\boldsymbol{\nu}}(\mathbf{x}, \boldsymbol{\xi})=\frac{1}{\sqrt{\mathrm{d}(\Gamma)}} \sum_{\boldsymbol{\theta} \in \Gamma_{\nu}^{\dagger}} \hat{b}(\boldsymbol{\theta}, \boldsymbol{\xi}) e^{i \boldsymbol{\theta} \mathbf{x}}=\frac{1}{\sqrt{\mathrm{d}(\Gamma)}} \sum_{0 \neq n \in \mathbb{Z}} \hat{b}(n \boldsymbol{\nu}, \boldsymbol{\xi}) e^{i n \boldsymbol{\nu} \mathbf{x}} .
$$

Clearly, $\left(b_{\boldsymbol{\nu}}\right)_{\boldsymbol{\nu}}=b_{\boldsymbol{\nu}}$. Notice that this symbol is symmetric if so is the initial symbol $b(\mathbf{x}, \boldsymbol{\xi})$, see $(2.6)$. Besides, $b_{\boldsymbol{\nu}} \in \mathbf{S}_{\alpha}(w, \Gamma)$ and $\left|b_{\boldsymbol{\nu}}\right|_{l, s}^{(\alpha)} \leq|b|_{l, s}^{(\alpha)}$. Let $\mathcal{C} \subset \mathbb{R}^{d}$ be a set, containing along with each point $\boldsymbol{\xi} \in \mathcal{C}$ the straight line $\{\boldsymbol{\xi}+t \boldsymbol{\nu}, t \in \mathbb{R}\}$. Then assuming that all the PDO's at hand are defined on the set $\mathrm{B}\left(\mathbb{R}^{d}\right.$ ) (see $(2.5)$ ), it is straightforward to see $\mathcal{H}(\mathcal{C})$ is a reducing subspace of $\operatorname{Op}\left(b_{\boldsymbol{\nu}}\right)$, and in particular, that $\mathcal{P}(\mathcal{C}) \mathrm{Op}\left(b_{\boldsymbol{\nu}}\right)=\mathrm{Op}\left(b_{\boldsymbol{\nu}}\right) \mathcal{P}(\mathcal{C})$ (see Subsect. 2.4 for definition of $\mathcal{P}(\mathcal{C})$ ). For example, the set $\Omega_{\boldsymbol{\nu}}=\Omega_{\boldsymbol{\nu}}(s)$ defined in (3.13) possesses this property and therefore $\mathcal{P}\left(\Omega_{\boldsymbol{\nu}}\right) \operatorname{Op}\left(b_{\boldsymbol{\nu}}\right)=\mathrm{Op}\left(b_{\boldsymbol{\nu}}\right) \mathcal{P}\left(\Omega_{\boldsymbol{\nu}}\right)$.

Below we decompose any symbol in the sum of symbols of the form $b_{\boldsymbol{\nu}}$. To explain how it is done we need to recall the definition of a primitive vector.

Definition 4.3 A non-zero vector $\nu \in \Lambda$ is said to be a primitive vector of the lattice $\Lambda \subset \mathbb{R}^{d}$ if

(i) The first non-zero coordinate of $\boldsymbol{\nu}=\left(\nu_{1}, \nu_{2}, \ldots, \nu_{d}\right)$ is positive;

(ii) There are no vector $\boldsymbol{\eta} \in \Lambda$ distinct from $\boldsymbol{\nu}$ and no integer $n>0$ such that $\boldsymbol{\nu}=n \boldsymbol{\eta}$.

It follows from this definition that for each non-zero vector $\chi \in \Lambda$ there exist a uniquely defined integer $n$ and a primitive vector $\boldsymbol{\nu} \in \Lambda$ such that $\chi=n \boldsymbol{\nu}$. Also, every two primitive vectors are linearly independent. The set of all primitive vectors of the lattice $\Lambda$ is denoted by $P \Lambda$. Now we can decompose any symbol $b \in \mathbf{S}_{\alpha}(w, \Gamma)$ into a sum over primitive vectors $\boldsymbol{\nu} \in P \Gamma^{\dagger}$ :

$$
b(\mathbf{x}, \boldsymbol{\xi})=b^{o}(\boldsymbol{\xi})+\sum_{\boldsymbol{\nu} \in P \Gamma^{\dagger}} b_{\boldsymbol{\nu}}(\mathbf{x}, \boldsymbol{\xi})
$$

see (4.9) for definition of $b^{o}$. From this and Definition (2.2) it follows that

$$
\max \left\{\left|b^{o}\right|_{l, s}^{(\alpha)}, \quad \mid b_{\boldsymbol{\eta}} \mathbf{I}_{l, s}^{(\alpha)}\right\} \leq|b|_{l, s}^{(\alpha)} \leq \max \left\{\left|b^{o}\right|_{l, s}^{(\alpha)}, \sup _{\boldsymbol{\nu} \in P \Gamma^{\dagger}}\left|b_{\boldsymbol{\nu}}\right|_{l, s}^{(\alpha)}\right\}, \quad \forall \boldsymbol{\eta} \in \Gamma^{\dagger} .
$$


Denote

$$
\widetilde{\Theta}_{r}=P \Gamma^{\dagger} \cap \Theta_{r} .
$$

Let us now combine two types of partition introduced above. Observe first that by definitions $(4.6)-(4.8)$ we always have $\left(b^{\natural}\right)_{\nu}=\left(b_{\boldsymbol{\nu}}\right)^{\natural}, \emptyset=\uparrow, \sharp, b$, so that one can use the notation $b_{\boldsymbol{\nu}}^{\natural}$ without risk of confusion. Moreover, since $b^{b}(\mathbf{x}, \boldsymbol{\xi})$ is a trigonometric polynomial, one can rewrite

$$
b^{b}(\mathbf{x}, \boldsymbol{\xi})=\sum_{\boldsymbol{\nu} \in \widetilde{\Theta}_{r}} b_{\nu}^{b}(\mathbf{x}, \boldsymbol{\xi}) .
$$

Due to the presence of a cut-off in the definition, the operators $b_{\nu}^{b}$ have an additional reducing subspace. Let $\Lambda_{\boldsymbol{\nu}}$ be the set defined in (3.12). Then the following lemma holds:

Lemma 4.4 Let the operator $B$ with the symbol $b \in \mathbf{P}_{\alpha}(w)$ be defined on $\mathrm{B}\left(\mathbb{R}^{d}\right)$ (see (2.5)). Suppose that $r \leq L$. Then $\left(B \mathcal{P}\left(\Lambda_{\boldsymbol{\nu}}\right)\right)^{b}=B_{\boldsymbol{\nu}}^{b} \mathcal{P}\left(\Lambda_{\boldsymbol{\nu}}\right)=B_{\boldsymbol{\nu}}^{b}$ for any $\boldsymbol{\nu} \in \Theta_{r}$.

For a symmetric symbol $b$ this lemma implies that the subspace $\mathcal{H}\left(\Lambda_{\nu}\right)$ is reducing for the operator $B_{\nu}^{b}$ and that $B_{\nu}^{b} \mathcal{P}\left(\mathbb{R}^{2} \backslash \Lambda_{\nu}\right)=0$.

Proof. It suffices to check that the support of $\zeta_{\boldsymbol{\theta}}, \boldsymbol{\theta}=n \boldsymbol{\nu} \in \Theta_{r}$ is contained in the domain $\Lambda_{\nu}$. By Definition (4.1), under the condition $r \leq L$ we have for each $\boldsymbol{\xi} \in \operatorname{supp} \zeta_{\boldsymbol{\theta}}$ and $n \neq 0$ :

$$
|\langle\boldsymbol{\theta}, \boldsymbol{\xi}\rangle| \leq|\langle\boldsymbol{\theta}, \boldsymbol{\xi}+\boldsymbol{\theta} / 2\rangle|+\frac{1}{2}|\boldsymbol{\theta}|^{2} \leq \frac{1}{2}(L|\boldsymbol{\theta}|+r|\boldsymbol{\theta}|) \leq L|\boldsymbol{\theta}| .
$$

It remains to recall $(3.12)$.

Suppose now that the symbol $b$ is symmetric. As was already mentioned, the subspace $\mathcal{H}\left(\Omega_{\boldsymbol{\nu}}\right)$ is then reducing for $B_{\boldsymbol{\nu}}$. Together with Lemma 4.4 this implies that for the set

$$
\hat{\Lambda}_{\nu}(s)=\Lambda_{\nu} \cap \Omega_{\nu}(s)
$$

the subspace $\mathcal{H}\left(\hat{\Lambda}_{\nu}\right)$ is reducing for $B_{\nu}^{b}$ if $r \leq L$. The properties of the operators $B_{\nu}^{b}$ on these reducing subspaces will be a key ingredient in our study of the density of states. In this study the set $\Lambda=\cup_{\boldsymbol{\nu} \in \Theta_{r}} \Lambda_{\boldsymbol{\nu}}$ plays the role of the resonant set described in the introduction.

First of all we need to establish some geometric properties of the sets $\hat{\Lambda}_{\nu}$. This will be done for the case $d=2$ only.

\subsection{Some geometric estimates for $d=2$}

Recall the notation $\mathbf{n}(\boldsymbol{\theta})=\boldsymbol{\theta}|\boldsymbol{\theta}|^{-1}$, and $\mathbf{t}^{\perp}=\mathbf{J t}$, see (3.14) for definition of $\mathbf{J}$.

Lemma 4.5 Let $d=2$. Suppose that $\boldsymbol{\theta}, \boldsymbol{\eta} \in \Theta_{r}$ are linearly independent. Then

$$
\left|\left\langle\mathbf{n}(\boldsymbol{\theta}), \mathbf{n}^{\perp}(\boldsymbol{\eta})\right\rangle\right| \geq \mathrm{d}\left(\Gamma^{\dagger}\right) r^{-2} .
$$


Proof. Let $\gamma_{1}, \gamma_{2}$ be two basis vectors of the lattice $\Gamma^{\dagger}$, so that $\boldsymbol{\theta}=n_{1} \gamma_{1}+n_{2} \gamma_{2}$ and $\boldsymbol{\eta}=m_{1} \gamma_{1}+m_{2} \gamma_{2}$ with some integers $n_{1}, n_{2}, m_{1}, m_{2}$. Consequently,

$$
\left\langle\boldsymbol{\theta},(\boldsymbol{\eta})^{\perp}\right\rangle=n_{1} m_{2}\left\langle\boldsymbol{\gamma}_{1}, \boldsymbol{\gamma}_{2}^{\perp}\right\rangle+n_{2} m_{1}\left\langle\boldsymbol{\gamma}_{1}^{\perp}, \boldsymbol{\gamma}_{2}\right\rangle=\left(n_{1} m_{2}-n_{2} m_{1}\right)\left\langle\boldsymbol{\gamma}_{1}, \boldsymbol{\gamma}_{2}^{\perp}\right\rangle .
$$

Since $\boldsymbol{\theta}$ and $\boldsymbol{\eta}$ are linearly independent, the integer factor $n_{1} m_{2}-n_{2} m_{1}$ never vanishes, and thus

$$
\left|\left\langle\boldsymbol{\theta}, \boldsymbol{\eta}^{\perp}\right\rangle\right| \geq\left|\left\langle\mathbf{J} \gamma_{1}, \gamma_{2}\right\rangle\right|=\mathrm{d}\left(\Gamma^{\dagger}\right) .
$$

Since $|\boldsymbol{\theta}| \leq r$ and $|\boldsymbol{\eta}| \leq r$, we obtain (4.17).

Lemma 4.6 Let $d=2$. Suppose that $\boldsymbol{\nu}, \boldsymbol{\mu} \in \Theta_{r}$ are linearly independent. If

$$
\mathrm{d}\left(\Gamma^{\dagger}\right) \rho r^{-2} L^{-1} \geq 4 \text { and } \rho L_{1}^{-1} \geq 4
$$

then one has

$$
|\langle\mathbf{n}(\boldsymbol{\mu}), \boldsymbol{\xi}\rangle| \geq 2^{-1} \mathrm{~d}\left(\Gamma^{\dagger}\right) r^{-2} \rho, \quad \forall \boldsymbol{\xi} \in \hat{\Lambda}_{\boldsymbol{\nu}}\left(\rho-L_{1}\right) .
$$

Moreover, $\hat{\Lambda}_{\boldsymbol{\nu}}\left(\rho-L_{1}\right) \cap \Lambda_{\boldsymbol{\mu}}(L)=\emptyset$ and $\operatorname{dist}\left(\hat{\Lambda}_{\boldsymbol{\nu}}\left(\rho-L_{1}\right), \Lambda_{\boldsymbol{\mu}}(L)\right) \geq 4^{-1} \mathrm{~d}\left(\Gamma^{\dagger}\right) r^{-2} \rho$.

Proof. Use the short-hand notation $\mathbf{n}_{\boldsymbol{\mu}}=\mathbf{n}(\boldsymbol{\mu}), \mathbf{n}_{\boldsymbol{\nu}}=\mathbf{n}(\boldsymbol{\nu})$. Decompose the vector $\mathbf{n}_{\boldsymbol{\mu}}$ into a sum as follows

$$
\begin{aligned}
\mathbf{n}_{\boldsymbol{\mu}} & =\left\langle\mathbf{n}_{\boldsymbol{\mu}}, \mathbf{n}_{\boldsymbol{\nu}}\right\rangle \mathbf{n}_{\boldsymbol{\nu}}+\left\langle\mathbf{n}_{\boldsymbol{\mu}}, \mathbf{n}_{\nu}^{\perp}\right\rangle \mathbf{n}_{\nu}^{\perp} \\
& =a \mathbf{n}_{\boldsymbol{\nu}}+a^{\perp} \mathbf{n}_{\nu}^{\perp}
\end{aligned}
$$

Then

$$
\left\langle\mathbf{n}_{\boldsymbol{\mu}}, \boldsymbol{\xi}\right\rangle=a^{\perp}\left\langle\mathbf{n}_{\boldsymbol{\nu}}^{\perp}, \boldsymbol{\xi}\right\rangle+a\left\langle\mathbf{n}_{\boldsymbol{\nu}}, \boldsymbol{\xi}\right\rangle .
$$

If $\boldsymbol{\xi} \in \hat{\Lambda}_{\boldsymbol{\nu}}=\hat{\Lambda}_{\boldsymbol{\nu}}\left(\rho-L_{1}\right)$ then $\left|\left\langle\mathbf{n}_{\boldsymbol{\nu}}, \boldsymbol{\xi}\right\rangle\right| \leq L$ and by $(3.15),\left|\left\langle\mathbf{n}_{\nu}^{\perp}, \boldsymbol{\xi}\right\rangle\right| \geq \rho-L_{1}$, so that

$$
\left|\left\langle\mathbf{n}_{\boldsymbol{\mu}}, \boldsymbol{\xi}\right\rangle\right| \geq\left|a^{\perp}\right|\left(\rho-L_{1}\right)-L .
$$

By Lemma $4.5\left|a^{\perp}\right| \geq \varkappa r^{-2}$ with $\varkappa=\mathrm{d}\left(\Gamma^{\dagger}\right)$, and hence

$$
\left|\left\langle\mathbf{n}_{\boldsymbol{\mu}}, \boldsymbol{\xi}\right\rangle\right| \geq \varkappa r^{-2} \rho-\left(\varkappa r^{-2} L_{1}+L\right)=\varkappa r^{-2} \rho\left(1-L_{1} \rho^{-1}-\varkappa^{-1} L r^{2} \rho^{-1}\right) .
$$

Since $\rho r^{-2} L^{-1} \geq 4 \varkappa^{-1}$ and $\rho L_{1} \geq 4$, the r.h.s. is greater than $2^{-1} \varkappa \rho r^{-2}$, which guarantees (4.18). The r.h.s. of (4.18) is also greater than $L$, which leads to the identity $\hat{\Lambda}_{\nu} \cap \Lambda_{\boldsymbol{\mu}}=\emptyset$. Also, for any $\boldsymbol{\xi} \in \hat{\Lambda}_{\boldsymbol{\nu}}$ and $\mathbf{t} \in \Lambda_{\boldsymbol{\mu}}$, we have

$$
|\boldsymbol{\xi}-\mathbf{t}| \geq\left|\left\langle\boldsymbol{\xi}, \mathbf{n}_{\boldsymbol{\mu}}\right\rangle\right|-\left|\left\langle\mathbf{t}, \mathbf{n}_{\boldsymbol{\mu}}\right\rangle\right| \geq 2^{-1} \varkappa \rho r^{-2}-L .
$$

Again, under the condition imposed on $\rho, r, L$ we have the required lower bound for the distance between $\hat{\Lambda}_{\nu}$ and $\Lambda_{\boldsymbol{\mu}}$. 


\subsection{Separation of variables}

Observe that the condition $d=2$ is crucial for the previous two lemmas. It guarantees that the sets $\hat{\Lambda}_{\nu}$ and $\Lambda_{\boldsymbol{\mu}}$ do not intersect for linearly independent $\boldsymbol{\nu}$ and $\boldsymbol{\mu}$. This fact allows one to "separate variables" when studying the model operator

$$
A=A^{o}+B^{b}, \quad A^{o}=\mathrm{Op}\left(a^{o}\right), a^{o}(\boldsymbol{\xi})=|\boldsymbol{\xi}|^{2}+b^{o}(\boldsymbol{\xi}) .
$$

Lemma 4.7 Let $d=2$. Suppose that $b \in \mathbf{P}_{0}(w)$ is a symmetric symbol. Let $\lambda_{0} \geq 0$ be a fixed number. Suppose that

$$
\begin{gathered}
1 \leq r \leq L, \\
\rho L_{1}^{-1} \geq 4, \\
\mathrm{~d}\left(\Gamma^{\dagger}\right) \rho r^{-2} L^{-1} \geq 4, \\
2 \rho L_{1} \geq \lambda_{0}+3 L^{2}+L_{1}^{2},
\end{gathered}
$$

and that $\left\|B^{o}\right\|+\left\|B^{b}\right\| \leq 2 L^{2}$. Then

$$
D(\lambda ; A)=D\left(\lambda ; A^{o} ; \mathbb{R}^{2} \backslash \hat{\Lambda}\right)+\sum_{\boldsymbol{\nu} \in \widetilde{\Theta}_{r}} D\left(\lambda ; A^{o}+B_{\nu}^{b} ; \hat{\Lambda}_{\nu}\right)
$$

for all $\lambda \geq \rho^{2}-\lambda_{0}$. Here $\hat{\Lambda}_{\nu}=\hat{\Lambda}_{\nu}\left(\rho-L_{1}\right)$ and $\hat{\Lambda}=\cup_{\nu} \hat{\Lambda}_{\nu}$.

Proof. Let $\mathcal{C}_{\nu}=\Lambda_{\nu} \backslash \hat{\Lambda}_{\nu}$ and

$$
\mathcal{C}=\cup_{\nu} \mathcal{C}_{\nu}, \Lambda=\cup_{\nu} \Lambda_{\nu}, \hat{\Lambda}=\cup_{\nu} \hat{\Lambda}_{\nu}
$$

By Lemma 4.4 the subspace $\mathcal{H}(\Lambda)$ is invariant for the operator $A$, and $A \mathcal{P}\left(\mathbb{R}^{2} \backslash \Lambda\right)=$ $A^{\circ} \mathcal{P}\left(\mathbb{R}^{2} \backslash \Lambda\right)$. By virtue of Lemma 4.6 the subspaces $\mathcal{H}(\hat{\Lambda})$ and $\mathcal{H}(\mathcal{C})$ are also invariant for $A$ and thus

$$
N(\lambda, A(\mathbf{k}))=N\left(\lambda, A^{o}(\mathbf{k}) ; \mathbb{R}^{2} \backslash \Lambda\right)+N(\lambda, A(\mathbf{k}) ; \mathfrak{C})+N(\lambda, A(\mathbf{k}) ; \hat{\Lambda}) .
$$

Furthermore, the third term in the last formula equals

$$
\sum_{\boldsymbol{\nu} \in \widetilde{\Theta}_{r}} N\left(\lambda, A^{o}(\mathbf{k})+B_{\nu}^{b}(\mathbf{k}) ; \hat{\Lambda}_{\nu}\right)
$$

since $\hat{\Lambda}_{\nu}$ are disjoint for distinct $\boldsymbol{\nu}$ 's. Hence it remains to verify that

$$
N(\lambda, A(\mathbf{k}) ; \mathcal{C})=N\left(\lambda, A^{o}(\mathbf{k}) ; \mathcal{C}\right) .
$$

For any $\boldsymbol{\xi} \in \mathcal{C}_{\nu}$ one has:

$$
|\boldsymbol{\xi}|^{2}=|\langle\boldsymbol{\xi}, \mathbf{n}(\boldsymbol{\nu})\rangle|^{2}+\left|\left\langle\boldsymbol{\xi}, \mathbf{n}^{\perp}(\boldsymbol{\nu})\right\rangle\right|^{2} \leq L^{2}+\left(\rho-L_{1}\right)^{2}=\rho^{2}-2 \rho L_{1}+L_{1}^{2}+L^{2} .
$$

Since $\rho L_{1}^{-1} \geq 4$ and $2 \rho L_{1} \geq \lambda_{0}+3 L^{2}+L_{1}^{2}$, the r.h.s. is bounded from above by $\rho^{2}-\lambda_{0}-2 L^{2} \leq \lambda-2 L^{2}$. Consequently, $\left\|A^{o} \mathcal{P}(\mathcal{C})\right\| \leq \lambda-2 L^{2}+\left\|B^{o}\right\|$. By virtue of the condition $\left\|B^{o}\right\|+\left\|B^{b}\right\| \leq 2 L^{2}$ we have $\|A \mathcal{P}(\mathcal{C})\| \leq \lambda$, which implies by an elementary perturbation argument that $N(\lambda, A(\mathbf{k}) ; \mathrm{C})=N\left(\lambda, A^{o}(\mathbf{k}) ; \mathrm{C}\right)$, as required. 


\section{Asymptotics in the invariant subspaces}

In this section we study the density of states for the model operator (4.19) introduced in the previous section. Our strategy is dictated by Lemma 4.7: since the sets $\hat{\Lambda}_{\boldsymbol{\nu}}$ do not intersect for distinct $\boldsymbol{\nu} \in \widetilde{\Theta}_{r}$, the investigation of each term in the r.h.s. of (4.20) is done independently. Furthermore, the symbol of the operator $A^{o}+B_{\nu}^{b}$ depends only on the projection $\langle\mathbf{x}, \mathbf{n}(\boldsymbol{\nu})\rangle$ (see (4.8)), so that in each subspace $\mathcal{H}\left(\hat{\Lambda}_{\nu}\right)$ the problem reduces to a one-dimensional one.

We begin with studying the operator $A^{o}+B_{\nu}^{b}$. Emphasize that the analysis of this operator is carried out without any restrictions on the dimension $d \geq 2$. On the contrary, in the closing subsection, dealing with the operator (4.19), we need to assume that $d=2$ in order to use Lemma 4.7 .

\subsection{A new class of symbols}

In order to describe the reduction to a one-dimensional problem we need to introduce a new class of symbols that encode the "one-dimensionality".

Let $z=z(\mathbf{x}, \eta), \mathbf{x} \in \mathbb{R}^{d}, \eta \in \mathbb{R}$ be a function and $L \geq 1$ be a constant. We say that this function belongs to the class $\mathbf{T}_{\alpha}(L, \Gamma), \alpha \in \mathbb{R}$, if it is $\Gamma$-periodic, $\mathrm{C}^{\infty}$-smooth in $\eta$ and its Fourier coefficients satisfy the condition

$$
\left|\partial_{\eta}^{s} \hat{z}(\boldsymbol{\theta}, \eta)\right| \leq C_{l, s}\langle\boldsymbol{\theta}\rangle^{-l} L^{\alpha-s},
$$

for all integer $l \geq 0$ and $s \geq 0$. Similarly to the class $\mathbf{S}_{\alpha}(L, \Gamma)$ introduce the norm

$$
\|z\|_{l, s}^{(\alpha)}=\max _{r \leq s} \max _{p \leq l} \sup _{\boldsymbol{\theta}} \sup _{\eta}\langle\boldsymbol{\theta}\rangle^{p} L^{-\alpha+r}\left|\partial_{\eta}^{r} \hat{z}(\boldsymbol{\theta}, \eta)\right| .
$$

We are interested in the PDO's with symbols having the following Fourier coefficients:

$$
\hat{b}(\boldsymbol{\theta}, \boldsymbol{\xi})=\left\{\begin{array}{l}
\hat{z}(\boldsymbol{\theta},\langle\boldsymbol{\xi}, \mathbf{n}(\boldsymbol{\theta})\rangle), \boldsymbol{\theta} \neq \mathbf{0}, \\
0, \boldsymbol{\theta}=\mathbf{0}
\end{array}\right.
$$

Clearly, $b \in \mathbf{S}_{\alpha}(L, \Gamma)$ and

$$
C_{l, s}^{-1}\|z\|_{l, s}^{(\alpha)} \leq|b|_{l, s}^{(\alpha)} \leq C_{l, s}\|z\|_{l, s}^{(\alpha)},
$$

with some constant $C_{l, s}$. We call the symbol $z$ symmetric if

$$
\hat{z}(\boldsymbol{\theta}, \eta)=\overline{\hat{z}(-\boldsymbol{\theta},-\eta-|\boldsymbol{\theta}|)}
$$

It is straightforward to check that under this condition the symbol (5.1) is symmetric in the sense of Definition (2.6). 


\subsection{Reduction to the case $d=1$}

Assuming, that the symbol $b$ is defined by (5.1) we study the density of states for an operator with the symbol

$$
\begin{aligned}
a(\mathbf{x}, \boldsymbol{\xi}) & =a^{o}(\boldsymbol{\xi})+b_{\boldsymbol{\nu}}(\mathbf{x}, \boldsymbol{\xi}), \\
a^{o}(\boldsymbol{\xi}) & =|\boldsymbol{\xi}|^{2}+f(\langle\boldsymbol{\xi}, \mathbf{n}(\boldsymbol{\nu})\rangle),
\end{aligned}
$$

with some $\mathbf{0} \neq \boldsymbol{\nu} \in \Gamma^{\dagger}$, and some real-valued uniformly bounded function $f$.

We are interested in the "partial" density of states $D(\lambda ; A ; \mathcal{C})$ with $\mathcal{C}=\Omega_{\nu}$ or $\mathcal{C}=\hat{\Lambda}_{\nu}$. The first step is to perform a change of variables which reduces $\Omega_{\nu}$ and $\hat{\Lambda}_{\nu}$ to $\Omega_{\mathbf{e}_{1}}$ and $\hat{\Lambda}_{\mathbf{e}_{1}}$. Let $\mathbf{M}=\mathbf{M}(\boldsymbol{\nu})$ be the orthogonal map from Subsect. 3.4, and let $W=W_{\mathbf{M}}$ be the unitary operator defined in (3.9). Then by Lemma 3.7 the symbol of $\tilde{A}=W^{*} A W$ is given by

$$
\tilde{a}(\mathbf{x}, \boldsymbol{\xi})=|\boldsymbol{\xi}|^{2}+f\left(\left\langle\mathbf{M}^{T} \boldsymbol{\xi}, \mathbf{n}(\boldsymbol{\nu})\right\rangle\right)+b_{\boldsymbol{\nu}}\left(\mathbf{M}^{-1} \mathbf{x}, \mathbf{M}^{T} \boldsymbol{\xi}\right) .
$$

Remembering that $\mathbf{M n}(\boldsymbol{\nu})=\mathbf{e}_{1}$, we have $\left\langle\mathbf{M}^{T} \boldsymbol{\xi}, \mathbf{n}(\boldsymbol{\nu})\right\rangle=\xi_{1}$. Using the last relation it is easy to find that $b_{\boldsymbol{\nu}}\left(\mathbf{M}^{-1} \mathbf{x}, \mathbf{M}^{T} \boldsymbol{\xi}\right)=\tilde{b}\left(x_{1}, \xi_{1}\right)$ with the symbol $\tilde{b}$ given by

$$
\tilde{b}(x, \eta)=\frac{1}{\sqrt{\mathrm{d}(\Gamma)}} \sum_{l \neq 0} \hat{z}\left(l \boldsymbol{\nu}, l|l|^{-1} \eta\right) e^{i|\boldsymbol{\nu}| l x}, x \in \mathbb{R}, \eta \in \mathbb{R} .
$$

Consequently,

$$
\begin{cases}\tilde{a}(\mathbf{x}, \boldsymbol{\xi})= & \tilde{a}^{o}(\boldsymbol{\xi})+\tilde{b}\left(x_{1}, \xi_{1}\right), \\ \tilde{a}^{o}(\boldsymbol{\xi})= & |\hat{\boldsymbol{\xi}}|^{2}+\xi_{1}^{2}+f\left(\xi_{1}\right), \hat{\boldsymbol{\xi}}=\left(\xi_{2}, \xi_{3}, \ldots, \xi_{d}\right) .\end{cases}
$$

Note that the symbol $\tilde{b}$ is periodic in $x$ with the period

$$
\tau=\tau(\boldsymbol{\nu})=2 \pi \frac{[|\boldsymbol{\nu}|]+1}{|\boldsymbol{\nu}|}
$$

so that the whole symbol $\tilde{a}$ is periodic w.r.t. the lattice $\Lambda=(\tau \mathbb{Z}) \times(2 \pi \mathbb{Z})^{d-1}$. Note that $\tau(\boldsymbol{\nu})$ is bounded from above and below uniformly in $\boldsymbol{\nu} \in P \Gamma^{\dagger}$. By Lemma 3.8 we have

$$
D_{\Gamma}(\lambda ; A ; \mathcal{C})=D_{\tilde{\Gamma}}(\lambda ; \tilde{A} ; \mathbf{M C}), \tilde{\Gamma}=\mathbf{M} \Gamma .
$$

Since the density of states does not depend on the lattice (see Subsection 2.4), we can replace $\tilde{\Gamma}$ with $\Lambda$ defined above, and thus

$$
D_{\Gamma}(\lambda ; A ; \mathcal{C})=D_{\Lambda}(\lambda ; \tilde{A} ; \mathbf{M} \mathcal{C}), \Lambda=(\tau \mathbb{Z}) \times(2 \pi \mathbb{Z})^{d-1} .
$$

In the next three lemmas we show that up to a controllable error the densities of states for the operator $A=\mathrm{Op}(a)$ and $A^{o}=\mathrm{Op}\left(a^{o}\right)$ coincide. We begin with a reduction to a one-dimensional operator $T$ with the symbol

$$
t(x, \eta)=\eta^{2}+f(\eta)+\tilde{b}(x, \eta), \quad x \in \mathbb{R}, \eta \in \mathbb{R} .
$$


The following lemma compares the densities of states for the operators $A=A^{\circ}+B_{\boldsymbol{\nu}}$ and $A^{o}+B_{\nu}^{b}$ with those for the operators $T$ and $T^{o}+T^{b}$ respectively, see subsection 4.1 for definitions.

Lemma 5.1 Suppose that $z \in \mathbf{T}_{\alpha}(L, \Gamma)$ is a symmetric symbol. Let $b$ be a symbol defined as in (5.1), and let $f$ be a real-valued uniformly bounded function on $\mathbb{R}$. Let $\tilde{b}$ and $t$ be the symbols defined in (5.5) and (5.9) respectively. Then

(i) The symbol $\tilde{b}$ belongs to $\mathbf{S}_{\alpha}(L, \tau \mathbb{Z})$ with $\tau$ specified in (5.7), it is symmetric, and $\mathbf{|} \tilde{b}_{l, s}^{(\alpha)} \leq C_{l, s} \boldsymbol{\|} z \boldsymbol{\|}_{l, s}^{(\alpha)}$ with a constant $C_{l, s}$ depending only on the lattice $\Gamma$;

(ii) For all $\lambda \in \mathbb{R}$ and $s \geq 0$ one has

$$
\left\{\begin{array}{l}
D\left(\lambda ; A ; \Omega_{\boldsymbol{\nu}}(s)\right)=\sigma D_{d-3}\left(\lambda-s^{2}, \lambda, T\right), \\
D\left(\lambda ; A^{o}+B_{\boldsymbol{\nu}}^{b} ; \Omega_{\boldsymbol{\nu}}(s)\right)=\sigma D_{d-3}\left(\lambda-s^{2}, \lambda ; T^{o}+T^{b}\right),
\end{array} \quad \sigma=\frac{\omega_{d-2}}{2(2 \pi)^{d-1}},\right.
$$

where

$$
\omega_{p}=(p+1) \mathrm{w}_{p+1}, p \geq 1,
$$

is the surface area of a unit sphere in $\mathbb{R}^{p+1}, \omega_{0}=2$, and the quantity $D_{q}$ is defined in (3.4).

Proof. (i) The $\tau$-periodicity of the symbol $\tilde{b}$ with the specified $\tau$ has already been observed. The estimate for the norm $|\tilde{b}|$ follows by inspection. The symmetry of $\tilde{b}$ follows from (5.3).

(ii) To prove (5.10) we use Lemma 3.9:

$$
\mathbf{M} \Omega_{\boldsymbol{\nu}}(s)=\Omega_{\mathbf{e}_{1}}(s)=\mathbb{R} \times\left\{\hat{\boldsymbol{\xi}} \in \mathbb{R}^{d-1}:|\hat{\boldsymbol{\xi}}| \geq s\right\} .
$$

Thus we can now use (5.8) and Lemma 2.6 to conclude that

$$
\begin{aligned}
(2 \pi)^{d-1} D_{\Gamma} & \left(\lambda, A ; \Omega_{\boldsymbol{\nu}}(s)\right)=(2 \pi)^{d-1} D_{\Lambda}\left(\lambda, \tilde{A} ; \Omega_{\mathbf{e}_{1}}(s)\right) \\
& =\int_{|\hat{\boldsymbol{\xi}}| \geq s} D\left(\lambda-|\hat{\boldsymbol{\xi}}|^{2} ; T\right) d \hat{\boldsymbol{\xi}}=\omega_{d-2} \int_{\xi \geq s} D\left(\lambda-\xi^{2} ; T\right) \xi^{d-2} d \xi \\
& =\frac{\omega_{d-2}}{2} \int_{-\infty}^{\lambda-s^{2}} D(\mu ; T)(\lambda-\mu)^{\frac{d-3}{2}} d \mu .
\end{aligned}
$$

By Lemma 4.2 an analogous formula holds for operators $A^{o}+B_{\nu}^{b}$ and $T^{o}+T^{b}$. Definition (3.4) leads to the proclaimed formula (5.10).

Before calculating the asymptotics of the r.h.s. of (5.10) we need to study the density of states for the operator $A^{o}=\operatorname{Op}\left(a^{o}\right)$ with the symbol $a^{o}$ defined in (5.4). We shall need the notation $\mathcal{E}(\lambda ; \cdot)$ introduced in $(2.13)$. 
Lemma 5.2 Let $f$ be a uniformly bounded function. Suppose that $s \geq 0$ and $\lambda \geq 0$ and $0 \leq \lambda_{0} \leq \lambda / 2$ are numbers such that

$$
\begin{gathered}
s^{2} \leq \lambda-\lambda_{0}-L^{2}-\sup _{\eta}|f(\eta)|, \\
L^{2}+\sup _{\eta}|f(\eta)| \leq \frac{\lambda-\lambda_{0}}{2} .
\end{gathered}
$$

Then for any $\lambda^{\prime}, \lambda^{\prime \prime} \in\left[\lambda-\lambda_{0}, \lambda+\lambda_{0}\right]$

$$
\left|D\left(\lambda^{\prime}, A^{o} ; \hat{\Lambda}_{\nu}(s)\right)-D\left(\lambda^{\prime \prime}, A^{o} ; \hat{\Lambda}_{\nu}(s)\right)\right| \leq C \lambda^{\frac{d-3}{2}}\left|\lambda^{\prime}-\lambda^{\prime \prime}\right| L,
$$

with a constant $C$ independent of the numbers $\lambda, \lambda^{\prime}, \lambda^{\prime \prime}, L, s$, vector $\boldsymbol{\nu}$ and symbol $f$.

Proof. Let $\mathbf{M}=\mathbf{M}(\boldsymbol{\nu})$ be the orthogonal map from Subsection 3.4. By Definition (4.16) and Lemma 3.9

$$
\mathbf{M} \hat{\Lambda}_{\nu}(s)=\hat{\Lambda}_{\mathbf{e}_{1}}(s)=\left\{\left|\xi_{1}\right| \leq L\right\} \times\{|\hat{\boldsymbol{\xi}}| \geq s\} .
$$

According to (5.8), formula (5.6) and Proposition 2.4, under the condition (5.12) for any $\lambda^{\prime} \in\left[\lambda-\lambda_{0}, \lambda+\lambda_{0}\right]$ we have the following formula:

$$
\begin{gathered}
(2 \pi)^{d} D\left(\lambda^{\prime}, A^{o} ; \hat{\Lambda}_{\boldsymbol{\nu}}(s)\right)=(2 \pi)^{d} D\left(\lambda^{\prime}, \tilde{A}^{o} ; \hat{\Lambda}_{\mathbf{e}_{1}}(s)\right)=\operatorname{vol}\left(\hat{\Lambda}_{\mathbf{e}_{1}}(s) \cap \mathcal{E}\left(\lambda^{\prime} ; \tilde{a}^{o}\right)\right) \\
=\mathrm{w}_{d-1} \int_{-L}^{L}\left[\lambda^{\prime}-\eta^{2}-f(\eta)\right]^{\frac{d-1}{2}} d \eta-2 L \mathrm{w}_{d-1} s^{d-1} .
\end{gathered}
$$

Here $\mathrm{w}_{p}$ is the volume of the unit ball in $\mathbb{R}^{p}$. Under the conditions (5.12) and (5.13) the above formula yields (5.14).

The next lemma yields an important intermediate result - it provides an asymptotic formula for the density of states of the operator $A^{o}+B_{\nu}^{b}$ :

Lemma 5.3 Let $f$ be a uniformly bounded function, and let $b$ be defined by (5.1) with a symmetric symbol $z \in \mathbf{T}_{0}(L, \Gamma)$. Suppose that $r \leq L$ and that for some $\lambda_{0} \in[0, \lambda / 2]$

$$
\left\{\begin{array}{l}
\left\|B_{\nu}^{b}\right\| \leq L^{2}, \sup _{\eta}|f(\eta)| \leq L^{2}, \\
3 L^{2} \leq \lambda-\lambda_{0}-s^{2}, s^{2} \geq \frac{\lambda+\lambda_{0}}{2} .
\end{array}\right.
$$

Then for all $\lambda^{\prime}, \lambda^{\prime \prime} \in\left[\lambda-\lambda_{0}, \lambda+\lambda_{0}\right]$ one has

$$
\left|D\left(\lambda^{\prime}, A^{o}+B_{\nu}^{b} ; \hat{\Lambda}_{\nu}(s)\right)-\frac{1}{(2 \pi)^{d}} \operatorname{vol}\left(\hat{\Lambda}_{\nu}(s) \cap \mathcal{E}\left(\lambda^{\prime} ; a^{o}\right)\right)\right| \leq C \lambda^{\frac{d-5}{2}} L,
$$

and

$$
\begin{aligned}
\mid D\left(\lambda^{\prime} ; A^{o}+B_{\nu}^{b} ; \hat{\Lambda}_{\nu}(s)\right)-D\left(\lambda^{\prime \prime} ; A^{o}+B_{\nu}^{b} ;\right. & \left.\hat{\Lambda}_{\nu}(s)\right) \mid \\
& \leq C \lambda^{\frac{d-3}{2}} L\left(\left|\lambda^{\prime}-\lambda^{\prime \prime}\right|+\lambda^{-1}\right)
\end{aligned}
$$


The constants in bounds (5.16) and (5.17) depend on $\lambda_{0}$. They do not depend on $\lambda, s, \nu, L, f$, and are uniform in the symbol $z$.

Proof. We derive the required formulas from the relation (5.10) with the use of Lemma 3.6. Let us check that its conditions are satisfied. The symbol $t^{b}=\tilde{b}^{b}=$ $\tilde{b}^{b}(x, \eta)$ (see $(5.9)$ for definition of $t$ ) has the form considered in Lemma 3.6 with $g=1$. Moreover, since $r<L$, by Lemma 4.4 we have $\mathcal{P}(\ell) \operatorname{Op}\left(\tilde{b}^{b}\right)=\operatorname{Op}\left(\tilde{b}^{b}\right)$, where $\ell=[-L, L]$. Furthermore, by Lemma $4.1 \tilde{b}^{b} \in \mathbf{S}_{0}(\langle\eta\rangle, \tau \mathbb{Z})$, and in view of Lemma $5.1(\mathrm{i})$ we have

$$
\mathbf{|} \tilde{b}^{b} \mathbf{|}_{l, s ;\langle\eta\rangle}^{(\alpha)} \leq C \mid \tilde{b} \mathbf{l}_{l, s ; L}^{(\alpha)} \leq \tilde{C}\|z\|_{l, s}^{(\alpha)},
$$

with some universal constants. And finally, the conditions (5.15) guarantee that $\|\mathrm{Op}(f)\| \leq L^{2},\left\|T^{b}\right\| \leq L^{2}$ and $\lambda^{\prime} / 2 \geq \lambda^{\prime}-s^{2} \geq 3 L^{2}$, for all $\lambda^{\prime} \in\left[\lambda-\lambda_{0}, \lambda+\lambda_{0}\right]$. Now we can apply Lemma 3.6 with $p=d-3$ to the r.h.s. of (5.10), which leads to

$$
\left|D_{d-3}\left(\lambda^{\prime}-s^{2}, \lambda^{\prime} ; T^{o}+T^{b}\right)-D_{d-3}\left(\lambda^{\prime}-s^{2}, \lambda^{\prime} ; T^{o}\right)\right| \leq C \lambda^{\prime \frac{d-5}{2}} L \leq \tilde{C} \lambda^{\frac{d-5}{2}} L,
$$

and hence

$$
\left|D\left(\lambda^{\prime}, A^{o}+B_{\nu}^{b} ; \Omega_{\nu}(s)\right)-D\left(\lambda^{\prime}, A^{o} ; \Omega_{\nu}(s)\right)\right| \leq C^{\prime} \lambda^{\frac{d-5}{2}} L,
$$

for all $\lambda^{\prime} \in\left[\lambda-\lambda_{0}, \lambda+\lambda_{0}\right]$. To establish a similar estimate for the set $\hat{\Lambda}_{\nu}(s)$ note that by Lemma 4.4

$$
D\left(\lambda^{\prime}, A^{o}+B_{\nu}^{b} ; \hat{\Lambda}_{\nu}(s)\right)=D\left(\lambda^{\prime} ; A^{o}+B_{\nu}^{b} ; \Omega_{\boldsymbol{\nu}}(s)\right)-D\left(\lambda^{\prime}, A^{o} ; \Omega_{\boldsymbol{\nu}}(s) \backslash \hat{\Lambda}_{\boldsymbol{\nu}}(s)\right),
$$

and hence (5.18) yields

$$
\left|D\left(\lambda^{\prime}, A^{o}+B_{\nu}^{b} ; \hat{\Lambda}_{\nu}(s)\right)-D\left(\lambda^{\prime}, A^{o} ; \hat{\Lambda}_{\nu}(s)\right)\right| \leq C^{\prime} \lambda^{\frac{d-5}{2}} L,
$$

for all $\lambda^{\prime} \in\left[\lambda-\lambda_{0}, \lambda+\lambda_{0}\right]$. By virtue of Proposition 2.4 the second term in the l.h.s. coincides with

$$
\frac{1}{(2 \pi)^{d}} \operatorname{vol}\left(\Omega_{\boldsymbol{\nu}}(s) \backslash \hat{\Lambda}_{\boldsymbol{\nu}}(s) \cap \mathcal{E}\left(\lambda^{\prime} ; a^{o}\right)\right),
$$

which implies (5.16).

Note that the conditions (5.15) guarantee (5.12) and (5.13). Now to obtain (5.17) it suffices to use (5.19) for $\lambda^{\prime}, \lambda^{\prime \prime}$, and (5.14).

\subsection{Density of states for the model operator (4.19)}

Our goal in this subsection is to establish a formula similar to (5.16) for the model operator (4.19). Now it is crucial to assume that $d=2$.

Let us first specify the symbols that we are working with. Let $f^{(\boldsymbol{\nu})}=f^{(\boldsymbol{\nu})}(\eta)$, $\eta \in \mathbb{R}, \boldsymbol{\nu} \in \widetilde{\Theta}_{r}$ be a collection of real-valued uniformly bounded functions. Also suppose that the quantity

$$
\varkappa_{\nu}=\sup _{|\eta| \geq 4 L}|\eta|^{\beta}\left|f^{(\boldsymbol{\nu})}(\eta)\right|
$$


is finite for some $\beta \geq 0$. This implies that

$$
\left|f^{(\boldsymbol{\nu})}(\eta)\right| \leq \varkappa_{\nu}|\eta|^{-\beta}, \forall|\eta| \geq 4 L .
$$

Instead of (5.4) assume that

$$
a^{o}(\boldsymbol{\xi})=|\boldsymbol{\xi}|^{2}+f(\boldsymbol{\xi}), f(\boldsymbol{\xi})=\sum_{\boldsymbol{\nu} \in \tilde{\Theta}_{r}} f^{(\boldsymbol{\nu})}(\langle\mathbf{n}(\boldsymbol{\nu}), \boldsymbol{\xi}\rangle) .
$$

All the subsequent results will be uniform in the function $f$ in the sense that they depend only on the constant $C$ in the bound $\sup _{\boldsymbol{\xi}}|f(\boldsymbol{\xi})| \leq C$.

The perturbation symbol $b$ is chosen in the same way as above, i.e., it is defined by the formula (5.1) for some symmetric $z \in \mathbf{T}_{0}(L, \Gamma)$. Our objective is to compare the density of states for the operator $A=A^{o}+B^{\mathrm{b}}$ with that of $A^{o}$. We are going to use the notation already exploited in the proof of Lemma 4.7:

$$
\Lambda=\cup_{\nu \in \widetilde{\Theta}_{r}} \Lambda_{\nu}, \hat{\Lambda}_{\nu}=\hat{\Lambda}_{\nu}\left(\rho-L_{1}\right), \hat{\Lambda}=\cup_{\nu \in \tilde{\Theta}_{r}} \hat{\Lambda}_{\nu} .
$$

For technical reasons we also need to include a bounded perturbation given by a self-adjoint $\mathrm{PDO} Q$ with a symbol $q \in \mathbf{P}_{0}(w)$ with an arbitrary weight $w$.

Theorem 5.4 Let $d=2$. Let the operator $A$ be as described above with $z \in \mathbf{T}_{0}(L, \Gamma)$, and let $q \in \mathbf{P}_{0}(w)$ be a symmetric symbol for some weight $w$. Denote

$$
\delta=\max _{\boldsymbol{\nu} \in \widetilde{\Theta}_{r}}\left\|Q_{\nu}^{b} \mathcal{P}\left(\hat{\Lambda}_{\nu}\right)\right\|, \quad \varkappa=\sum_{\boldsymbol{\nu} \in \tilde{\Theta}_{r}} \varkappa_{\nu} .
$$

For a fixed $\lambda_{0} \geq 0$ denote

$$
\lambda_{1}=\lambda_{0}+\delta+\varkappa(4 L)^{-\beta} .
$$

Suppose that $\rho^{2} \geq 16 \lambda_{1}$ and that

$$
\begin{aligned}
& \left\{\begin{array}{l}
1 \leq r \leq L, \\
\rho \geq 4 L_{1}, \\
\mathrm{~d}\left(\Gamma^{\dagger}\right) \rho r^{-2} \geq 8 L,
\end{array}\right. \\
& 2 \rho L_{1} \geq 2 \lambda_{1}+3 L^{2}+L_{1}^{2} .
\end{aligned}
$$

Then there exists a constant $L_{0}=L_{0}(z, q, f)$ such that under the condition

$$
L \geq L_{0},
$$

one has

$\left|D\left(\lambda ; A^{o}+B^{b}+Q^{b}\right)-\frac{1}{(2 \pi)^{2}} \operatorname{vol} \mathcal{E}\left(\lambda ; a^{o}\right)\right| \leq C r^{2} \rho^{-1} L\left(\varkappa r^{2 \beta} \rho^{-\beta}+\delta\right)+C r^{2} \rho^{-3} L$,

for all $\lambda \in\left[\rho^{2}-\lambda_{0}, \rho^{2}+\lambda_{0}\right]$. The constants $C, L_{0}$ are uniform in the symbols $f, q$ and $z$, and $C$ may depend on $\lambda_{0}$ and $\lambda_{1}$. 
Proof. Since $z \in \mathbf{T}_{0}(L)$ and $q \in \mathbf{P}_{0}(w)$, the operators $B, B^{b}, Q, Q^{b}$ are bounded by Proposition 3.1 and Lemma 5.1(i):

$$
\left\{\begin{array}{l}
\left\|B_{\nu}^{b}\right\|+\left\|B^{b}\right\|+\|B\| \leq C_{l}\|z\|_{l, 0}^{(0)}, \\
\left\|Q_{\nu}^{b}\right\|+\left\|Q^{b}\right\|+\|Q\| \leq C_{l}|q|_{l, 0}^{(0)},
\end{array} \quad \forall \boldsymbol{\nu} \in \Gamma^{\dagger}, l>2 .\right.
$$

Choosing sufficiently large $L_{0}$ one ensures that $\|\mathrm{Op}(f)\|+\left\|B^{\mathrm{b}}+Q^{\mathrm{b}}\right\| \leq 2 L^{2}$. Remembering also (5.23), (5.24), one guarantees that the conditions of Lemma 4.7 are fulfilled. Consequently, in view of (4.20)

$$
D\left(\lambda ; A^{o}+B^{b}+Q^{b}\right)=D\left(\lambda ; A^{o} ; \mathbb{R}^{2} \backslash \hat{\Lambda}\right)+\sum_{\nu \in \tilde{\Theta}_{r}} D\left(\lambda ; A^{o}+B_{\nu}^{b}+Q_{\nu}^{b} ; \hat{\Lambda}_{\nu}\right),
$$

for all $\lambda \geq \rho^{2}-\lambda_{1}$. By Proposition 2.4 the first term equals

$$
D\left(\lambda ; A^{o} ; \mathbb{R}^{2} \backslash \hat{\Lambda}\right)=\frac{1}{(2 \pi)^{2}} \operatorname{vol}\left(\mathbb{R}^{2} \backslash \hat{\Lambda} \cap \mathcal{E}\left(\lambda ; a^{o}\right)\right) .
$$

Let us consider each summand in the second term separately. Let us fix a $\boldsymbol{\nu} \in \widetilde{\Theta}_{r}$ and define

$$
y^{o}(\boldsymbol{\xi})=|\boldsymbol{\xi}|^{2}+f^{(\boldsymbol{\nu})}(\langle\mathbf{n}(\boldsymbol{\nu}), \boldsymbol{\xi}\rangle), Y^{o}=\mathrm{Op}\left(y^{o}\right) .
$$

Note that in view of (4.18) we have $|\langle\mathbf{n}(\boldsymbol{\mu}), \boldsymbol{\xi}\rangle| \geq 2^{-1} \mathrm{~d}\left(\Gamma^{\dagger}\right) r^{-2} \rho \geq 4 L$ for all $\boldsymbol{\xi} \in \hat{\Lambda}_{\boldsymbol{\nu}}$ for any two $\boldsymbol{\nu}, \boldsymbol{\mu} \in \widetilde{\Theta}_{r}$ such that $\boldsymbol{\nu} \neq \boldsymbol{\mu}$, so that by (5.20)

$$
\max _{\boldsymbol{\nu} \in \tilde{\Theta}_{r}} \sup _{\boldsymbol{\xi} \in \hat{\Lambda}_{\nu}}\left|\sum_{\boldsymbol{\nu} \neq \boldsymbol{\mu} \in \widetilde{\Theta}_{r}} f^{(\boldsymbol{\mu})}(\langle\mathbf{n}(\boldsymbol{\mu}), \boldsymbol{\xi}\rangle)\right| \leq 2^{\beta}\left(\mathrm{d}\left(\Gamma^{\dagger}\right)\right)^{-\beta} \varkappa r^{2 \beta} \rho^{-\beta} \leq \varkappa(4 L)^{-\beta} .
$$

Consequently, with $\delta_{\nu}=\left\|Q_{\nu}^{\text {b }} \mathcal{P}\left(\hat{\Lambda}_{\nu}\right)\right\|$ one has

$$
\begin{aligned}
Y^{o}+B_{\nu}^{b}-2^{\beta}\left(\mathrm{d}\left(\Gamma^{\dagger}\right)\right)^{-\beta} \varkappa r^{2 \beta} \rho^{-\beta}-\delta_{\boldsymbol{\nu}} & \leq A^{o}+B_{\nu}^{b}+Q_{\nu}^{b} \\
& \leq Y^{o}+B_{\nu}^{b}+2^{\beta}\left(\mathrm{d}\left(\Gamma^{\dagger}\right)\right)^{-\beta} \varkappa r^{2 \beta} \rho^{-\beta}+\delta_{\nu}
\end{aligned}
$$

Here we assume that all the operators are considered on their invariant subspace $\mathcal{H}\left(\hat{\Lambda}_{\boldsymbol{\nu}}\right)$. Choosing $L_{0}$ sufficiently large we may assume that $\left\|B_{\nu}^{\mathrm{b}}\right\| \leq L^{2}$, $\sup _{\eta}\left|f^{(\boldsymbol{\nu})}(\eta)\right| \leq L^{2}$, so that the first half of the conditions (5.15) are satisfied. Using the bounds $\rho^{2} \geq 16 \lambda_{1}$ and (5.24), under the condition $\lambda \in\left[\rho^{2}-\lambda_{1}, \rho^{2}+\lambda_{1}\right]$ (see (5.22) for definition of $\lambda_{1}$ ) one proves that the second half of (5.15) is also satisfied with $s=\rho-L_{1}$ and $\lambda_{1}$ instead of $\lambda_{0}$. Therefore, by (5.16)

$$
\begin{gathered}
\left|D\left(\lambda ; Y^{o}+B_{\nu}^{b} ; \hat{\Lambda}_{\nu}\right)-\frac{1}{(2 \pi)^{2}} \operatorname{vol}\left(\hat{\Lambda}_{\nu} \cap \mathcal{E}\left(\lambda ; y^{o}\right)\right)\right| \leq C \rho^{-3} L, \\
\forall \lambda \in\left[\rho^{2}-\lambda_{1}, \rho^{2}+\lambda_{1}\right] .
\end{gathered}
$$


In view of monotonicity of the density of states (see Proposition 2.1) and the bounds (5.28), (5.17) we have

$$
\begin{gathered}
\left|D\left(\lambda ; Y^{o}+B_{\nu}^{b} ; \hat{\Lambda}_{\nu}\right)-D\left(\lambda ; A^{o}+B_{\nu}^{b}+Q_{\nu}^{b} ; \hat{\Lambda}_{\nu}\right)\right| \leq C \rho^{-3} L+C^{\prime} \rho^{-1} L\left(\varkappa r^{2 \beta} \rho^{-\beta}+\delta\right), \\
\forall \lambda \in\left[\rho^{2}-\lambda_{0}, \rho^{2}+\lambda_{0}\right] .
\end{gathered}
$$

The last two estimates lead to the bound

$$
\begin{gathered}
\left|D\left(\lambda ; A^{o}+B_{\nu}^{b}+Q_{\nu}^{b} ; \hat{\Lambda}_{\nu}\right)-\frac{1}{(2 \pi)^{2}} \operatorname{vol}\left(\hat{\Lambda}_{\nu} \cap \mathcal{E}\left(\lambda ; y^{o}\right)\right)\right| \\
\leq C \rho^{-3} L+C^{\prime} \rho^{-1} L\left(\varkappa r^{2 \beta} \rho^{-\beta}+\delta\right), \\
\forall \lambda \in\left[\rho^{2}-\lambda_{0}, \rho^{2}+\lambda_{0}\right] .
\end{gathered}
$$

Using this estimate for $Q=B=0$, in combination with Proposition 2.4 one also concludes that

$$
\left|\operatorname{vol}\left(\hat{\Lambda}_{\nu} \cap \mathcal{E}\left(\lambda ; a^{o}\right)\right)-\operatorname{vol}\left(\hat{\Lambda}_{\nu} \cap \mathcal{E}\left(\lambda ; y^{o}\right)\right)\right| \leq C \rho^{-3} L+C^{\prime} \rho^{-1} L \varkappa r^{2 \beta} \rho^{-\beta} .
$$

This shows that in the estimate (5.29) the set $\mathcal{E}\left(\lambda ; y^{\circ}\right)$ can be replaced with $\mathcal{E}\left(\lambda ; a^{o}\right)$. Adding together the formulae (5.29) for all $\boldsymbol{\nu} \in \tilde{\Theta}_{r}$, taking into account that card $\tilde{\Theta}_{r} \leq C r^{2}$, we obtain that

$$
\begin{aligned}
& \mid \sum_{\nu \in \tilde{\Theta}_{r}} D\left(\lambda ; A^{o}+B_{\nu}^{b}+Q_{\nu}^{b} ; \hat{\Lambda}_{\nu}\right)-\frac{1}{(2 \pi)^{2}} \operatorname{vol}\left(\hat{\Lambda} \cap \mathcal{E}\left(\lambda ; a^{o}\right)\right) \mid \\
& \leq C r^{2} \rho^{-3} L+C^{\prime} r^{2} \rho^{-1} L\left(\varkappa r^{2 \beta} \rho^{-\beta}+\delta\right) .
\end{aligned}
$$

It remains to combine the obtained formula with (5.27), using (5.26).

\section{A "gauge transformation"}

In this and all the subsequent sections we use the notation $\mathbf{S}_{\alpha}$ for the class $\mathbf{S}_{\alpha}(L)$. For the classes $\mathbf{S}_{\alpha}(w)$ with different weight $w$ we use the full notation to avoid confusion.

\subsection{Preparation}

Our strategy will be to find a unitary operator which reduces an elliptic PDO $H=H_{0}+\operatorname{Op}(b)$ (see Definition (2.8)) with $b \in \mathbf{S}_{\alpha}(\langle\boldsymbol{\xi}\rangle), \alpha<m$ to another PDO, whose symbol, up to some controllable small errors, depends only on $\boldsymbol{\xi}$. Very soon we shall focus on the operators of second order, but in this subsection the order is irrelevant and it is allowed to be any positive $m>0$. The sought unitary operator will be constructed in the form $U=e^{i \Psi}$ with a suitable bounded self-adjoint $\Gamma$ periodic PDO $\Psi$. This is why we sometimes call it a "gauge transformation". It is useful to consider $e^{i \Psi}$ as an element of the group

$$
U(t)=\exp \{i \Psi t\}, \quad \forall t \in \mathbb{R} .
$$


We assume that the operator $\operatorname{ad}\left(H_{0}, \Psi\right)$ is bounded, so that $U(t) D\left(H_{0}\right)=D\left(H_{0}\right)$. This assumption will be justified later on. Let us express the operator

$$
A_{t}=U(-t) H U(t)
$$

via its (weak) derivative w.r.t. $t$ :

$$
A_{t}=H+\int_{0}^{t} U(-\tau) \operatorname{ad}(H ; \Psi) U(\tau) d \tau
$$

By induction it is easy to show that

$$
\begin{aligned}
A_{1} & =H+\sum_{j=1}^{M} \frac{1}{j !} \operatorname{ad}^{j}(H ; \Psi)+R_{M+1}^{(1)}, \\
R_{M+1}^{(1)} & =\int_{0}^{1} d \tau_{1} \int_{0}^{\tau_{1}} d \tau_{2} \ldots \int_{0}^{\tau_{M}} U\left(-\tau_{M+1}\right) \operatorname{ad}^{M+1}(H ; \Psi) U\left(\tau_{M+1}\right) d \tau_{M+1} .
\end{aligned}
$$

The operator $\Psi$ is sought in the form

$$
\Psi=\sum_{k=1}^{N} \Psi_{k}, \Psi_{k}=\mathrm{Op}\left(\psi_{k}\right), \psi_{k} \in \mathbf{S}_{k(\alpha-m)+1} .
$$

Substitute this formula in (6.1) and rewrite, regrouping the terms:

$$
\begin{aligned}
A_{1}= & H_{0}+B+\sum_{j=1}^{M} \frac{1}{j !} \sum_{l=j}^{M} \sum_{k_{1}+k_{2}+\cdots+k_{j}=l} \operatorname{ad}\left(H ; \Psi_{k_{1}}, \Psi_{k_{2}}, \ldots, \Psi_{k_{j}}\right) \\
& +R_{M+1}^{(1)}+R_{M+1}^{(2)}, \\
R_{M+1}^{(2)}= & \sum_{j=1}^{M} \frac{1}{j !} \sum_{k_{1}+k_{2}+\cdots+k_{j} \geq M+1} \operatorname{ad}\left(H ; \Psi_{k_{1}}, \Psi_{k_{2}}, \ldots, \Psi_{k_{j}}\right) .
\end{aligned}
$$

Rewrite:

$$
\begin{aligned}
A_{1}= & H_{0}+B+\sum_{l=1}^{M} \operatorname{ad}\left(H_{0} ; \Psi_{l}\right)+\sum_{j=2}^{M} \frac{1}{j !} \sum_{l=j}^{M} \sum_{k_{1}+k_{2}+\cdots+k_{j}=l} \operatorname{ad}\left(H_{0} ; \Psi_{k_{1}}, \Psi_{k_{2}}, \ldots, \Psi_{k_{j}}\right) \\
& +\sum_{j=1}^{M} \frac{1}{j !} \sum_{l=j}^{M} \sum_{k_{1}+k_{2}+\cdots+k_{j}=l} \operatorname{ad}\left(B ; \Psi_{k_{1}}, \Psi_{k_{2}}, \ldots, \Psi_{k_{j}}\right)+R_{M+1}^{(1)}+R_{M+1}^{(2)} .
\end{aligned}
$$

Switch the summation signs:

$$
\begin{aligned}
A_{1}= & H_{0}+B+\sum_{l=1}^{M} \operatorname{ad}\left(H_{0} ; \Psi_{l}\right)+\sum_{l=2}^{M} \sum_{j=2}^{l} \frac{1}{j !} \sum_{k_{1}+k_{2}+\cdots+k_{j}=l} \operatorname{ad}\left(H_{0} ; \Psi_{k_{1}}, \Psi_{k_{2}}, \ldots, \Psi_{k_{j}}\right) \\
& +\sum_{l=2}^{M+1} \sum_{j=1}^{l-1} \frac{1}{j !} \sum_{k_{1}+k_{2}+\cdots+k_{j}=l-1} \operatorname{ad}\left(B ; \Psi_{k_{1}}, \Psi_{k_{2}}, \ldots, \Psi_{k_{j}}\right)+R_{M+1}^{(1)}+R_{M+1}^{(2)} .
\end{aligned}
$$


Introduce the notation

$$
\begin{gathered}
B_{1}=B, \\
B_{l}=\sum_{j=1}^{l-1} \frac{1}{j !} \sum_{k_{1}+k_{2}+\cdots+k_{j}=l-1} \operatorname{ad}\left(B ; \Psi_{k_{1}}, \Psi_{k_{2}}, \ldots, \Psi_{k_{j}}\right), l \geq 2, \\
T_{l}=\sum_{j=2}^{l} \frac{1}{j !} \sum_{k_{1}+k_{2}+\cdots+k_{j}=l} \operatorname{ad}\left(H_{0} ; \Psi_{k_{1}}, \Psi_{k_{2}}, \ldots, \Psi_{k_{j}}\right), l \geq 2 .
\end{gathered}
$$

We emphasize that the operators $B_{l}$ and $T_{l}$ depend only on $\Psi_{1}, \Psi_{2}, \ldots, \Psi_{l-1}$. One more rearrangement:

$$
\begin{gathered}
A_{1}=H_{0}+B+\sum_{l=1}^{M} \operatorname{ad}\left(H_{0}, \Psi_{l}\right)+\sum_{l=2}^{M} B_{l}+\sum_{l=2}^{M} T_{l}+R_{M+1}, \\
R_{M+1}=B_{M+1}+R_{M+1}^{(1)}+R_{M+1}^{(2)} .
\end{gathered}
$$

Now we can specify our algorithm for finding $\Psi_{k}$ 's. The symbols $\psi_{k}$ will be found from the following system of commutator equations:

$$
\begin{gathered}
\operatorname{ad}\left(H_{0} ; \Psi_{1}\right)+B_{1}^{\sharp}=0, \\
\operatorname{ad}\left(H_{0} ; \Psi_{l}\right)+B_{l}^{\sharp}+T_{l}^{\sharp}=0, l \geq 2,
\end{gathered}
$$

and hence

$$
\left\{\begin{array}{l}
A_{1}=A_{0}+X_{M}^{b, \uparrow}+R_{M+1} \\
X_{M}=\sum_{l=1}^{M} B_{l}+\sum_{l=2}^{M} T_{l} \\
A_{0}=H_{0}+\sum_{l=1}^{M} B_{l}^{o}+\sum_{l=2}^{M} T_{l}^{o} .
\end{array}\right.
$$

Below, in Lemma 6.3 we shall prove that all the symbols $b_{l}$ and $t_{l}$ belong to appropriate classes $\mathbf{S}_{\beta}$ with some $\beta$, and thus by (4.10) the symbols $b_{l}^{\sharp}, t_{l}^{\sharp}$ possess the same property. This means that $B_{l}^{\sharp}$ and $T_{l}^{\sharp}$ are bounded (see Proposition 3.1) and hence the commutators $\operatorname{ad}\left(H_{0}, \Psi_{l}\right)$ are also bounded in view of $(6.7),(6.8)$. This justifies the assumption that $\operatorname{ad}\left(H_{0}, \Psi\right)$ is bounded, made in the beginning of the formal calculations in this Section.

\subsection{Commutator equations}

Since our primary concern is the Schrödinger operator, from now on we assume that $m=2$ and $\mathbf{F}=\mathbf{I}$ in Definition (2.8). Before proceeding to the study of the commutator equations $(6.7),(6.8)$ note that the symbol

$$
\tau_{\boldsymbol{\theta}}(\boldsymbol{\xi})=h_{0}(\boldsymbol{\xi}+\boldsymbol{\theta})-h_{0}(\boldsymbol{\xi})=2\langle\boldsymbol{\theta}, \boldsymbol{\xi}+\boldsymbol{\theta} / 2\rangle
$$


satisfies the bound

$$
\left|\mathbf{D}_{\boldsymbol{\xi}}^{\mathbf{s}} \tau_{\theta}^{-1}\right| \leq C_{s}|\boldsymbol{\theta}|^{-1} L^{-s-1}, \boldsymbol{\theta} \neq 0,
$$

for all $\boldsymbol{\xi}$ in the support of the function $\varphi_{\boldsymbol{\theta}}$ (see (4.2)). This estimate will come in handy in the next lemma.

Lemma 6.1 Let $A=\operatorname{Op}(a)$ be a symmetric PDO such that $a \in \mathbf{S}_{\alpha}$. Then the PDO $\Psi$ with the Fourier coefficients of the symbol $\psi(\mathbf{x}, \boldsymbol{\xi})$ given by

$$
\hat{\psi}(\boldsymbol{\theta}, \boldsymbol{\xi})=i \frac{\hat{a}^{\sharp}(\boldsymbol{\theta}, \boldsymbol{\xi})}{\tau_{\boldsymbol{\theta}}(\boldsymbol{\xi})},
$$

solves the equation

$$
\operatorname{ad}\left(H_{0} ; \Psi\right)+\mathrm{Op}\left(a^{\sharp}\right)=0 .
$$

Moreover, the operator $\Psi$ is bounded and self-adjoint, $\psi \in \mathbf{S}_{\alpha-1}$ and

$$
\left|\psi \mathbf{|}_{l, s}^{(\alpha-1)} \leq C\right| a \mathbf{|}_{l-1, s}^{(\alpha)} .
$$

The constant $C$ is independent of the parameter $L \geq 1$ and the symbol a.

Proof. Let $t$ be the symbol of $\operatorname{ad}\left(H_{0} ; \Psi\right)$. The Fourier transform $\hat{t}(\boldsymbol{\theta}, \boldsymbol{\xi})$ is easy to find using (3.2):

$$
\hat{t}(\boldsymbol{\theta}, \boldsymbol{\xi})=i\left(h_{0}(\boldsymbol{\xi}+\boldsymbol{\theta})-h_{0}(\boldsymbol{\xi})\right) \hat{\psi}(\boldsymbol{\theta}, \boldsymbol{\xi})=i \tau_{\boldsymbol{\theta}}(\boldsymbol{\xi}) \hat{\psi}(\boldsymbol{\theta}, \boldsymbol{\xi}) .
$$

Therefore the equation (6.13) amounts to

$$
i \tau_{\boldsymbol{\theta}}(\boldsymbol{\xi}) \hat{\psi}(\boldsymbol{\theta}, \boldsymbol{\xi})=-\hat{a}^{\sharp}(\boldsymbol{\theta}, \boldsymbol{\xi})=-\hat{a}(\boldsymbol{\theta}, \boldsymbol{\xi}) \varphi_{\boldsymbol{\theta}}(\boldsymbol{\xi} ; L) .
$$

By definition of the function $\varphi_{\boldsymbol{\theta}}$, a solution $\hat{\psi}$ exists and is given by (6.12). This symbol satisfies the condition (2.6), so that $\Psi$ is a symmetric operator. Note also that by (4.4) and (6.11) the symbol $\psi$ belongs to $\mathbf{S}_{\alpha-1}$ and one easily shows that

$$
|\psi| \mathbf{l}_{l, s}^{(\alpha-1)} \leq C \mid a \mathbf{|}_{l-1, s}^{(\alpha)} .
$$

This estimate for $s=0$ and Proposition 3.1 ensure the boundedness of $\Psi$.

Remark 6.2 Let the symbols $a$ and $\psi$ be as in Lemma 6.1 and consider the commutator $\operatorname{Op}(\mathfrak{a})=\operatorname{ad}(\mathrm{Op}(g), \Psi)$ with some symmetric symbol $g \in \mathbf{S}_{\gamma}$. By (3.2)

$$
\begin{aligned}
\hat{\mathfrak{a}}(\boldsymbol{\chi}, \boldsymbol{\xi}) & =\frac{i}{\sqrt{\mathrm{d}(\Gamma)}} \sum_{\boldsymbol{\theta}+\boldsymbol{\phi}=\boldsymbol{\chi}}[\hat{g}(\boldsymbol{\phi}, \boldsymbol{\xi}+\boldsymbol{\theta}) \hat{\psi}(\boldsymbol{\theta}, \boldsymbol{\xi})-\hat{g}(\boldsymbol{\theta}, \boldsymbol{\xi}) \hat{\psi}(\boldsymbol{\phi}, \boldsymbol{\xi}+\boldsymbol{\theta})] \\
& =-\frac{1}{\sqrt{\mathrm{d}(\Gamma)}} \sum_{\boldsymbol{\theta}+\boldsymbol{\phi}=\boldsymbol{\chi}}\left[\frac{\hat{g}(\boldsymbol{\phi}, \boldsymbol{\xi}+\boldsymbol{\theta}) \hat{a}^{\sharp}(\boldsymbol{\theta}, \boldsymbol{\xi})}{\tau_{\boldsymbol{\theta}}(\boldsymbol{\xi})}-\frac{\hat{g}(\boldsymbol{\phi}, \boldsymbol{\xi}) \hat{a}^{\sharp}(\boldsymbol{\theta}, \boldsymbol{\xi}+\boldsymbol{\phi})}{\tau_{\boldsymbol{\theta}}(\boldsymbol{\xi}+\boldsymbol{\phi})}\right] .
\end{aligned}
$$


Analogously, one can easily derive a formula for the commutator symbol $\mathfrak{a}_{\mu, \boldsymbol{\eta}}=$ $\operatorname{ad}\left(g_{\boldsymbol{\mu}}, \psi_{\boldsymbol{\eta}}\right)$ with arbitrary $\boldsymbol{\mu}, \boldsymbol{\eta} \in \Gamma^{\dagger}$, see Subsection 4.2 for definition of these symbols. It is the same formula as above, but with the summation restricted to appropriate subsets of the lattice $\Gamma^{\dagger}$ :

$$
\hat{\mathfrak{a}}_{\boldsymbol{\mu}, \boldsymbol{\eta}}(\boldsymbol{\chi}, \boldsymbol{\xi})=-\frac{1}{\sqrt{\mathrm{d}(\Gamma)}} \sum_{\substack{\boldsymbol{\theta}+\boldsymbol{\phi}=\chi \\ \boldsymbol{\theta} \in \Gamma_{\eta}^{\dagger}, \boldsymbol{\phi} \in \Gamma_{\mu}^{\dagger}}}\left[\frac{\hat{g}(\boldsymbol{\phi}, \boldsymbol{\xi}+\boldsymbol{\theta}) \hat{a}^{\sharp}(\boldsymbol{\theta}, \boldsymbol{\xi})}{\tau_{\boldsymbol{\theta}}(\boldsymbol{\xi})}-\frac{\hat{g}(\boldsymbol{\phi}, \boldsymbol{\xi}) \hat{a}^{\sharp}(\boldsymbol{\theta}, \boldsymbol{\xi}+\boldsymbol{\phi})}{\tau_{\boldsymbol{\theta}}(\boldsymbol{\xi}+\boldsymbol{\phi})}\right],
$$

see (4.12) for definition of $\Gamma_{\nu}^{\dagger}$. Recalling that $\tau_{-\boldsymbol{\theta}}(\boldsymbol{\xi}+\boldsymbol{\theta})=-\tau_{\boldsymbol{\theta}}(\boldsymbol{\xi})$, and using the property (2.6) we obtain

$$
\begin{aligned}
\hat{t}(0, \boldsymbol{\xi}) & =-\frac{1}{\sqrt{\mathrm{d}(\Gamma)}} \sum_{\boldsymbol{\theta}}\left[\frac{\hat{g}(-\boldsymbol{\theta}, \boldsymbol{\xi}+\boldsymbol{\theta}) \hat{a}^{\sharp}(\boldsymbol{\theta}, \boldsymbol{\xi})}{\tau_{\boldsymbol{\theta}}(\boldsymbol{\xi})}-\frac{\hat{g}(\boldsymbol{\theta}, \boldsymbol{\xi}) \hat{a}^{\sharp}(-\boldsymbol{\theta}, \boldsymbol{\xi}+\boldsymbol{\theta})}{\tau_{-\boldsymbol{\theta}}(\boldsymbol{\xi}+\boldsymbol{\theta})}\right] \\
& =-\frac{1}{\sqrt{\mathrm{d}(\Gamma)}} \sum_{\boldsymbol{\theta}} \frac{1}{\tau_{\boldsymbol{\theta}}(\xi)}\left[\overline{\hat{g}(\boldsymbol{\theta}, \boldsymbol{\xi})} \hat{a}^{\sharp}(\boldsymbol{\theta}, \boldsymbol{\xi})+\hat{g}(\boldsymbol{\theta}, \boldsymbol{\xi}) \overline{\hat{a}^{\sharp}(\boldsymbol{\theta}, \boldsymbol{\xi})}\right] .
\end{aligned}
$$

Let us apply Lemma 6.1 to equations (6.7) and (6.8).

Lemma 6.3 Let $b \in \mathbf{S}_{\alpha}$ be a symmetric symbol. Then there exists a sequence of selfadjoint bounded PDO's $\Psi_{l}, l=1,2, \ldots$ with the symbols $\psi_{l} \in \mathbf{S}_{\beta_{l}}, \beta_{l}=l(\alpha-2)+1$, such that (6.7) and (6.8) hold, and

$$
\mid \psi_{l} \mathbf{|}_{r, s}^{\left(\beta_{l}\right)} \leq C\left(\mathbf{|} b \mathbf{|}_{p, n}^{(\alpha)}\right)^{l}, l \geq 1 ;
$$

(ii) The symbols $b_{l}, t_{l}$ of the corresponding operators $B_{l}, T_{l}$ belong to $\mathbf{S}_{\gamma_{l}}$ with $\gamma_{l}=l(\alpha-2)+2$ and

$$
\begin{aligned}
& \left|b_{l}\right|_{r, s}^{\left(\gamma_{l}\right)}+\left|t_{l}\right|_{r, s}^{\left(\gamma_{l}\right)} \leq C\left(|b|_{p, n}^{(\alpha)}\right)^{l}, l \geq 2 ; \\
& b_{2}^{o}(\boldsymbol{\xi})+t_{2}^{o}(\boldsymbol{\xi})=-\frac{1}{\mathrm{~d}(\Gamma)} \sum_{\boldsymbol{\theta} \in \Theta_{r}} \frac{|\hat{b}(\boldsymbol{\theta}, \boldsymbol{\xi})|^{2}}{\tau_{\boldsymbol{\theta}}(\boldsymbol{\xi})}\left(1-\zeta_{\boldsymbol{\theta}}^{2}(\boldsymbol{\xi} ; L)\right) .
\end{aligned}
$$

The constant $C$ in (6.15) and (6.16) does not depend on b, but depends on l, $r, s, \alpha$. The integer-valued parameters $p, n$ in (6.15) and (6.16) depend on $l, r, s, \alpha$.

(iv) If $|b|_{l, s}^{(\alpha)} \leq C_{l, s} L^{2-\alpha}$ for all $l$ and $s$, then for some positive integer $n, p$ the following bounds hold:

$$
\begin{gathered}
\left\|R_{M+1}\right\| \leq \tilde{C}\left(|b|_{p, n}^{(\alpha)}\right)^{M+1} L^{(M+1)(\alpha-2)+2}, \quad p=p(M, \alpha), n=n(M, \alpha) ; \\
\left|x_{M}\right|_{l, s}^{(\alpha)} \leq \tilde{C}|b|_{p, n}^{(\alpha)}, p=p(l, s, \alpha, M), \quad n=n(l, s, \alpha, M) .
\end{gathered}
$$

The constant $\tilde{C}$ depends only on the constants $C_{l, s}$ and the parameters $M, \alpha$. 
Proof. The existence of $\psi_{1} \in \mathbf{S}_{\beta_{1}}$ with required properties follows from Lemma 6.1. Further proof is by induction.

To make the calculations less cumbersome, throughout the proof we adopt the following notational convention. If two symbols, $\phi_{1}$ and $\phi_{2}$ satisfy the estimate $\left|\phi_{1}\right|_{l, s}^{(\beta)} \leq C\left|\phi_{2}\right|_{p, n}^{(\omega)}$ with some $p=p(l, s)$ and $n=n(l, s)$ we simply write $\left|\phi_{1}\right|^{(\beta)} \leq C\left|\phi_{2}\right|^{(\omega)}$.

Suppose that $\psi_{k}$ with $k=1,2, \ldots, K-1$ satisfy (6.15). In order to conclude that $\psi_{K}$ also satisfies (6.15), first we need to check that $b_{K}$ and $t_{K}$ satisfy (6.16). Step I. Estimates for $b_{l}$. To begin with we prove that all the symbols $b_{l}$ with $l \leq K$, satisfy the estimate (6.16). We first obtain a bound for $\operatorname{ad}\left(b ; \psi_{k_{1}} \psi_{k_{2}}, \ldots, \psi_{k_{j}}\right)$ with $k_{1}+k_{2}+\cdots+k_{j}=l-1$. To this end we use (6.15) and Proposition 3.4 to conclude that

$$
\mathbf{|} \operatorname{ad}\left(b ; \psi_{k_{1}}, \psi_{k_{2}}, \ldots, \psi_{k_{j}}\right) \mathbf{|}^{(\gamma)} \leq C \mid b \mathbf{|}^{(\alpha)} \prod_{n=1}^{j}\left(|b|^{(\alpha)}\right)^{k_{n}}=C\left(|b|^{(\alpha)}\right)^{l}
$$

with

$\gamma=\alpha+\sum_{n=1}^{j}\left(\beta_{k_{j}}-1\right)=\alpha+\sum_{n=1}^{j} k_{j}(\alpha-2)=(l-1)(\alpha-2)+\alpha-2+2=l(\alpha-2)+2$.

This implies that $b_{l}$ satisfies (6.16) for all $l \leq K$.

Step II. Estimates for $t_{l}$. For the symbols $t_{l}$ the proof is by induction. First of all, $\overline{\text { note that } \operatorname{ad}\left(h_{0} ; \psi_{1}, \psi_{1}\right)}=-\operatorname{ad}\left(b^{\sharp}, \psi_{1}\right)$, so that, by Proposition 3.4

$$
\mid \operatorname{ad}\left(h_{0} ; \psi_{1}, \psi_{1}\right) \mathbf{|}^{(2 \alpha-2)} \leq C\left(|\boldsymbol{|} b|^{(\alpha)}\right)^{2},
$$

and thus $t_{2}$ satisfies (6.16). Suppose that all $t_{k}$ with $k \leq l-1 \leq K-1$ satisfy (6.16). Then by Definition (6.8) and (4.10) all ad $\left(h_{0} ; \psi_{k}\right), k \leq l-1$, satisfy the same bound. Remembering that the definition of $t_{l}$ involves only $\psi_{k}$ with $k \leq l-1$, and applying Proposition 3.4, we obtain for $k_{1}+k_{2}+\cdots+k_{j}=l, j \geq 2$ :

$$
\mathbf{|} \operatorname{ad}\left(h_{0} ; \psi_{k_{1}}, \psi_{k_{2}}, \ldots, \psi_{k_{j}}\right) \mathbf{|}^{(\gamma)}=\mathbf{I} \operatorname{ad}\left(\operatorname{ad}\left(h_{0} ; \psi_{k_{1}}\right) ; \psi_{k_{2}}, \ldots, \psi_{k_{j}}\right) \mathbf{|}^{(\gamma)} \leq\left(\mid \boldsymbol{|} b \mathbf{|}^{(\alpha)}\right)^{l},
$$

with

$$
\gamma=k_{1}(\alpha-2)+2+\sum_{n=2}^{j}\left(k_{n}(\alpha-2)+1-1\right)=l(\alpha-2)+2 .
$$

This leads to (6.16) for all $t_{l}, l \leq K$.

Step III. To handle $\Psi_{K}$ we use the solution $\Psi$ of the equation (6.13) constructed in Lemma 6.1. Then from Definition (6.8) and steps I, II we immediately conclude that $\psi_{K} \in \mathbf{S}_{\gamma}$ with $\gamma=\beta-1, \beta=K(\alpha-2)+2$ and that

$$
\mid \psi_{K} \mathbf{|}^{(\beta)} \leq C\left(\left|\mathbf{|} b_{K} \mathbf{|}^{(\beta)}+\right| t_{K} \mathbf{|}^{(\beta)}\right) \leq C\left(|\mathbf{|} b|^{(\alpha)}\right)^{K},
$$

as required. 
Step IV. Proof of (iii). By (6.4) and by (6.5), (6.7)

$$
B_{2}=\operatorname{ad}\left(B ; \Psi_{1}\right), T_{2}=-\frac{1}{2} \operatorname{ad}\left(B^{\sharp} ; \Psi_{1}\right) .
$$

It follows from (6.12) that

$$
\hat{\psi}_{1}(\boldsymbol{\theta}, \boldsymbol{\xi})=i \frac{\hat{b}^{\sharp}(\boldsymbol{\theta}, \boldsymbol{\xi})}{\tau_{\theta}(\boldsymbol{\xi})} .
$$

Remark 6.2 and Definition (4.9) lead to the formulas

$$
b_{2}^{o}(\boldsymbol{\xi})=-\frac{2}{\mathrm{~d}(\Gamma)} \sum_{\boldsymbol{\theta} \in \Theta_{r}} \frac{|\hat{b}(\boldsymbol{\theta}, \boldsymbol{\xi})|^{2}}{\tau_{\boldsymbol{\theta}}(\boldsymbol{\xi})} \varphi_{\boldsymbol{\theta}}(\boldsymbol{\xi} ; L), t_{2}^{o}(\boldsymbol{\xi})=\frac{1}{\mathrm{~d}(\Gamma)} \sum_{\boldsymbol{\theta} \in \Theta_{r}} \frac{|\hat{b}(\boldsymbol{\theta}, \boldsymbol{\xi})|^{2}}{\tau_{\boldsymbol{\theta}}(\boldsymbol{\xi})}\left(\varphi_{\boldsymbol{\theta}}(\boldsymbol{\xi} ; L)\right)^{2} .
$$

Adding them up and recalling that $\varphi_{\boldsymbol{\theta}}=1-\zeta_{\boldsymbol{\theta}}$, we get (6.17).

Step V. Proof of (iv). The remainder $R_{M+1}$ (see (6.6)) consists of three components. In view of $(6.16), b_{M+1} \in \mathbf{S}_{(M+1)(\alpha-2)+2}$, so that by Remark 3.2 the norm of $B_{M+1}$ is bounded by $\left(|\boldsymbol{b}|^{(\alpha)}\right)^{M+1} L^{(M+1)(\alpha-2)+2}$ as required.

Consider now $R_{M+1}^{(1)}$ defined in (6.1). Let $\psi=\sum_{l=1}^{M} \psi_{l}$. Since $|b|^{(\alpha)} \leq$ $C L^{2-\alpha}$, according to $(6.15),(2.7)$ we have

$$
\left|\psi_{l} \mathbf{|}^{(\alpha-1)} \leq C L^{(l-1)(\alpha-2)}\left(\mid \boldsymbol{|} \mathbf{|}^{(\alpha)}\right)^{l} \leq C^{\prime}\right| \boldsymbol{b} \mathbf{|}^{(\alpha)} .
$$

Similarly, by Definition (6.9) we have $|X|^{(\alpha)} \leq C|b|^{(\alpha)}$ in view of (6.16), which proves (6.19). It follows from (6.7) and (6.8) that $\operatorname{ad}\left(H_{0}, \Psi\right)+X=0$. Now, repeating the same argument as on Steps 1 and II, we conclude that $\left.\left|\operatorname{ad}^{M+1}(H, \Psi)\right|\right|^{(\gamma)} \leq$ $\left(\left.|b|\right|^{(\alpha)}\right)^{M+1}$ with $\gamma=(M+1)(\alpha-2)+2$. By Remark 3.2 this leads to the required estimate for the norm $\left\|R_{M+1}^{(1)}\right\|$.

In the same way the norm of the error $R_{M+1}^{(2)}$ defined in (6.3) can be shown to satisfy the same bound. This completes the proof of (6.18).

\section{Density of states for operator $A_{1}$}

In this section we apply the transformation constructed in the previous section, to the Schrödinger operator, that is to the operator (2.8) with $m=2$ and $\mathbf{F}=\mathbf{I}$, $b(\mathbf{x}, \boldsymbol{\xi})=V(\mathbf{x})$, so that $\alpha=0$. For the proof of Theorem 2.3 we shall need the representation (6.9) with $M=2$.

We begin with deriving further consequences from Lemma 6.3. From now on we shall use the notation $V_{j}$ instead of $B_{j}, j=1,2, \ldots$ All the estimates below are uniform in $V$.

The majority of the results below are obtained for $d=2$, although for some intermediate results the condition $d \geq 2$ will be sufficient. 


\subsection{Operators $V_{2}^{b}$ and $T_{2}^{b}$}

Let us investigate in more detail the operators $V_{2}^{\mathrm{b}}$ and $T_{2}^{\mathrm{b}}$. Recall again that by (6.4), (6.5) and (6.7)

$$
V_{2}=\operatorname{ad}\left(V ; \Psi_{1}\right), T_{2}=-\frac{1}{2} \operatorname{ad}\left(V^{\sharp} ; \Psi_{1}\right) .
$$

We start by studying the symbols

$$
\mathfrak{a}_{\boldsymbol{\mu}, \boldsymbol{\eta}}=\operatorname{ad}\left(V_{\boldsymbol{\mu}},\left(\psi_{1}\right)_{\boldsymbol{\eta}}\right), \quad \mathfrak{b}_{\boldsymbol{\mu}, \boldsymbol{\eta}}=-\frac{1}{2} \operatorname{ad}\left(V_{\boldsymbol{\mu}}^{\sharp},\left(\psi_{1}\right)_{\boldsymbol{\eta}}\right)
$$

with $\boldsymbol{\mu}, \boldsymbol{\eta} \in P \Gamma^{\dagger}$, see Subsection 4.2 for definition of the symbols $b_{\boldsymbol{\nu}}$ and of the set of the primitive lattice vectors $P \Gamma^{\dagger}$. By (4.14), (4.10) and Lemma 6.3 used with $m=2, \alpha=0$, we have $V_{\boldsymbol{\mu}}, V_{\boldsymbol{\mu}}^{\sharp} \in \mathbf{S}_{0},\left(\psi_{1}\right)_{\boldsymbol{\eta}} \in \mathbf{S}_{-1}$, and

$$
\left|V_{\mu} \mathbf{|}_{l, s}^{(0)}+\right| V_{\mu}^{\sharp} \mathbf{I}_{l, s}^{(0)} \leq C_{l, s}\left|V \mathbf{|}_{l, 0}^{(0)}, \quad\right|\left(\psi_{1}\right)_{\eta} \mathbf{|}_{l, s}^{(-1)} \leq C_{l, s} \mid V \mathbf{|}_{p, 0}^{(0)}, p=p(l, s) .
$$

Consequently by Proposition $3.4 \mathfrak{a}_{\boldsymbol{\mu}, \boldsymbol{\eta}}, \mathfrak{b}_{\mu, \boldsymbol{\eta}} \in \mathbf{S}_{-2}$ and

$$
\left|\mathfrak{a}_{\boldsymbol{\mu}, \boldsymbol{\eta}} \mathbf{|}_{l, s}^{(-2)}+\right| \mathfrak{b}_{\boldsymbol{\mu}, \boldsymbol{\eta}} \mathbf{|}_{l, s}^{(-2)} \leq C_{l, s}\left(\mathbf{|} V \mathbf{|}_{p, 0}^{(0)}\right)^{2}, \quad p=p(l, s)
$$

uniformly in $\boldsymbol{\mu}, \boldsymbol{\eta} \in P \Gamma^{\dagger}$. We shall need more detailed properties of these commutators. In particular, let us find bounds for symbols $\left(\mathfrak{a}_{\mu, \boldsymbol{\eta}}\right)_{\nu}$ and $\left(\mathfrak{b}_{\mu, \boldsymbol{\eta}}\right)_{\boldsymbol{\nu}}, \boldsymbol{\nu} \in P \Gamma^{\dagger}$. In the next lemma and further on we shall need the explicit formulas for these symbols, which follow from (6.14) and (6.22). For brevity we write only the formula for $\hat{\mathfrak{a}}_{\mu, \eta}$ :

$$
\hat{\mathfrak{a}}_{\boldsymbol{\mu}, \boldsymbol{\eta}}(\boldsymbol{\chi}, \boldsymbol{\xi})=-\frac{1}{\sqrt{\mathrm{d}(\Gamma)}} \sum_{\substack{\boldsymbol{\phi}+\boldsymbol{\theta}=\chi \\ \boldsymbol{\phi} \in \mathrm{\Gamma}_{\mu}^{+}, \boldsymbol{\theta} \in \Gamma_{\eta}^{\dagger} \cap \Theta_{r}}} \hat{V}(\boldsymbol{\phi}) \hat{V}(\boldsymbol{\theta})\left[\frac{\varphi_{\boldsymbol{\theta}}(\boldsymbol{\xi} ; L)}{\tau_{\boldsymbol{\theta}}(\boldsymbol{\xi})}-\frac{\varphi_{\boldsymbol{\theta}}(\boldsymbol{\xi}+\boldsymbol{\phi} ; L)}{\tau_{\boldsymbol{\theta}}(\boldsymbol{\xi}+\boldsymbol{\phi})}\right] .
$$

In this formula $\chi \in \Gamma^{\dagger}$ and $\tau_{\boldsymbol{\theta}}$ is defined in (6.10). Recall the notation $\hat{\Lambda}_{\nu}=$ $\hat{\Lambda}_{\nu}\left(\rho-L_{1}\right)$ and (4.15).

Lemma 7.1 Let $d=2$. Let $V$ be as above and $\psi_{1}$ be as found in Lemma 6.3. Suppose that

$$
\left\{\begin{array}{l}
1 \leq r \leq L \\
\rho \geq 4 L_{1} \\
\mathrm{~d}\left(\Gamma^{\dagger}\right) \rho r^{-2} \geq 16 L
\end{array}\right.
$$

Then for any $\boldsymbol{\nu} \in \widetilde{\Theta}_{r}$ one has

$$
\sum_{\substack{\boldsymbol{\mu} \in P \Gamma^{\dagger}, \boldsymbol{\eta} \in \tilde{\Theta}_{r} \\ \boldsymbol{\mu} \neq \boldsymbol{\eta}}}\left(\mathbf{I}\left(\mathfrak{a}_{\boldsymbol{\mu}, \boldsymbol{\eta}}\right)_{\boldsymbol{\nu}}^{b} \chi\left(\cdot ; \hat{\Lambda}_{\boldsymbol{\nu}}\right) \mathbf{I}_{l, 0}^{(0)}+\mathbf{I}\left(\mathfrak{b}_{\boldsymbol{\mu}, \boldsymbol{\eta}}\right)_{\boldsymbol{\nu}}^{b} \chi\left(\cdot ; \hat{\Lambda}_{\boldsymbol{\nu}}\right) \mathbf{I}_{l, 0}^{(0)}\right) \leq C_{l}\left(\mathbf{|} V \mathbf{I}_{p, 0}^{(0)}\right)^{2} r^{4} \rho^{-2}
$$

for all $l \geq 0, p=p(l)$, uniformly in $\boldsymbol{\nu} \in \widetilde{\Theta}_{r}$. (Here $\chi\left(\cdot ; \hat{\Lambda}_{\nu}\right)$ denotes the multiplication by the function $\chi\left(\boldsymbol{\xi} ; \hat{\Lambda}_{\boldsymbol{\nu}}\right)$.) 
Proof. Let us estimate each term in the sum (7.4) individually. For the sake of brevity we conduct the proof only for the case of the symbol $\mathfrak{a}_{\boldsymbol{\mu}, \boldsymbol{\eta}}$. For this we use (7.2) with $\chi \in \Gamma_{\nu}^{\dagger}$, assuming that $\boldsymbol{\xi} \in \hat{\Lambda}_{\boldsymbol{\nu}}$. Since we are interested in the operator $\mathfrak{a}_{\boldsymbol{\mu}, \boldsymbol{\eta}}^{b}$, we may assume that $\chi \in \Theta_{r}$ (see Definition (4.8)), and hence we have $\phi \in \Theta_{2 r}$ in (7.2).

Let us estimate first the terms in the square brackets in (7.2). Since $\boldsymbol{\mu} \neq \boldsymbol{\eta}$, the vectors $\boldsymbol{\nu}$ and $\boldsymbol{\theta}, \boldsymbol{\phi}$ in (7.2) are pairwise linearly independent. Consequently, in view of (4.18) and (7.3) we have for $\boldsymbol{\xi} \in \hat{\Lambda}_{\nu}$ the bounds

and

$$
\begin{aligned}
|\langle\mathbf{n}(\boldsymbol{\theta}), \boldsymbol{\xi}\rangle| & \geq 2^{-1} \mathrm{~d}\left(\Gamma^{\dagger}\right) r^{-2} \rho \geq 8 L, \\
|\langle\mathbf{n}(\boldsymbol{\theta}), \boldsymbol{\xi}+\boldsymbol{\phi}\rangle| & \geq 2^{-1} \mathrm{~d}\left(\Gamma^{\dagger}\right) r^{-2} \rho-2 r \geq 6 L, \\
\left|\tau_{\boldsymbol{\theta}}(\boldsymbol{\xi})\right| & =2|\langle\boldsymbol{\theta}, \boldsymbol{\xi}+\boldsymbol{\theta} / 2\rangle| \\
& \geq|\boldsymbol{\theta}|\left(\mathrm{d}\left(\Gamma^{\dagger}\right) r^{-2} \rho-r\right) \geq \frac{1}{2}|\boldsymbol{\theta}| \mathrm{d}\left(\Gamma^{\dagger}\right) r^{-2} \rho, \\
\left|\tau_{\boldsymbol{\theta}}(\boldsymbol{\xi}+\boldsymbol{\phi})\right| & =2|\langle\boldsymbol{\theta}, \boldsymbol{\xi}+\boldsymbol{\phi}+\boldsymbol{\theta} / 2\rangle| \\
& \geq|\boldsymbol{\theta}|\left(\mathrm{d}\left(\Gamma^{\dagger}\right) r^{-2} \rho-5 r\right) \geq \frac{1}{2}|\boldsymbol{\theta}| \mathrm{d}\left(\Gamma^{\dagger}\right) r^{-2} \rho .
\end{aligned}
$$

By Definitions (4.1) and (4.2), in view of the above bounds we have

$$
\varphi_{\boldsymbol{\theta}}(\boldsymbol{\xi})=\varphi_{\boldsymbol{\theta}}(\boldsymbol{\xi}+\boldsymbol{\phi})=1, \forall \boldsymbol{\xi} \in \hat{\Lambda}_{\boldsymbol{\nu}},
$$

and hence for $\chi \in \Theta_{r} \cap \Gamma_{\nu}^{\dagger}, \boldsymbol{\xi} \in \hat{\Lambda}_{\boldsymbol{\nu}}$ the symbol $\hat{\mathfrak{a}}_{\boldsymbol{\mu}, \boldsymbol{\eta}}$ has the form:

$$
\hat{\mathfrak{a}}_{\boldsymbol{\mu}, \boldsymbol{\eta}}(\boldsymbol{\chi}, \boldsymbol{\xi})=-\frac{1}{\sqrt{\mathrm{d}(\Gamma)}} \sum_{\substack{\boldsymbol{\phi}+\boldsymbol{\theta}=\boldsymbol{\chi} \\ \boldsymbol{\phi} \in \Gamma_{\boldsymbol{\mu}}^{\dagger}, \boldsymbol{\theta} \in \Gamma_{\boldsymbol{\eta}}^{\dagger} \cap \Theta_{r}}} \hat{V}(\boldsymbol{\phi}) \hat{V}(\boldsymbol{\theta})\left[\frac{1}{\tau_{\boldsymbol{\theta}}(\boldsymbol{\xi})}-\frac{1}{\tau_{\boldsymbol{\theta}}(\boldsymbol{\xi}+\boldsymbol{\phi})}\right] .
$$

According to (7.5), (7.6),

$$
\left|\frac{1}{\tau_{\boldsymbol{\theta}}(\boldsymbol{\xi})}-\frac{1}{\tau_{\boldsymbol{\theta}}(\boldsymbol{\xi}+\boldsymbol{\phi})}\right|=\frac{2|\langle\boldsymbol{\theta}, \boldsymbol{\phi}\rangle|}{\tau_{\boldsymbol{\theta}}(\boldsymbol{\xi}) \tau_{\boldsymbol{\theta}}(\boldsymbol{\xi}+\boldsymbol{\phi})} \leq C|\phi| r^{4} \rho^{-2},
$$

and hence

$$
\begin{aligned}
\mathbf{|}\left(\mathfrak{a}_{\boldsymbol{\mu}, \boldsymbol{\eta}}^{b}\right)_{\boldsymbol{\nu}} \chi\left(\cdot ; \hat{\Lambda}_{\boldsymbol{\nu}}\right) \mathbf{|}_{l, 0}^{(0)} & \leq C \max _{\boldsymbol{\chi} \in \Theta_{r}} \max _{\boldsymbol{\xi} \in \hat{\Lambda}_{\boldsymbol{\nu}}}\langle\boldsymbol{\chi}\rangle^{l}\left|\hat{\mathfrak{a}}_{\boldsymbol{\mu}, \boldsymbol{\eta}}(\boldsymbol{\chi}, \boldsymbol{\xi})\right| \\
& \leq C_{l} r^{4} \rho^{-2} \sum_{\boldsymbol{\phi} \in \Gamma_{\boldsymbol{\mu}}^{\dagger}}|\boldsymbol{\phi}|^{l+1}|\hat{V}(\boldsymbol{\phi})| \sum_{\boldsymbol{\theta} \in \Gamma_{\boldsymbol{\eta}}^{\dagger}}|\boldsymbol{\theta}|^{l}|\hat{V}(\boldsymbol{\theta})| .
\end{aligned}
$$

The r.h.s. is finite since $V \in \mathbf{S}_{0}$. Summing these estimates over $\boldsymbol{\mu} \in P \Gamma^{\dagger}$ and $\boldsymbol{\eta} \in P \Gamma^{\dagger}$, we bound the r.h.s. by the product

$$
\sum_{\boldsymbol{\phi} \in \Gamma^{\dagger}}|\boldsymbol{\phi}|^{l+1}|\hat{V}(\boldsymbol{\phi})| \sum_{\boldsymbol{\theta} \in \Gamma^{\dagger}}|\boldsymbol{\theta}|^{l}|\hat{V}(\boldsymbol{\theta})| \leq C_{p}\left(|V|_{p, 0}^{(0)}\right)^{2}
$$

for any $p>l+3$. Consequently, the estimate $(7.4)$ is fulfilled. The symbol $\mathfrak{b}_{\boldsymbol{\mu}, \boldsymbol{\eta}}$ can be treated in the same way. These calculations are omitted to avoid repetitions. 

follows:

Using (4.13) for $V, V^{\sharp}$ and $\psi_{1}$, we can now decompose the symbol $V_{2}+t_{2}$ as

$$
\begin{aligned}
& V_{2}+t_{2}=V_{2}^{o}+t_{2}^{o}+\mathfrak{f}+\mathfrak{g}, \\
& \mathfrak{f}=\sum_{\boldsymbol{\nu} \in P \Gamma^{\dagger}}\left(\mathfrak{a}_{\boldsymbol{\nu}, \boldsymbol{\nu}}+\mathfrak{b}_{\boldsymbol{\nu}, \boldsymbol{\nu}}\right)_{\boldsymbol{\nu}}, \mathfrak{g}=\sum_{\boldsymbol{\nu} \in P \Gamma^{\dagger}} \sum_{\substack{\boldsymbol{\mu}, \boldsymbol{\eta} \in P^{\dagger} \\
\boldsymbol{\mu} \neq \boldsymbol{\eta}}}\left(\mathfrak{a}_{\boldsymbol{\mu}, \boldsymbol{\eta}}+\mathfrak{b}_{\boldsymbol{\mu}, \boldsymbol{\eta}}\right)_{\boldsymbol{\nu}} .
\end{aligned}
$$

Our objective is to show that the symbol $\mathfrak{f}$ has the form (5.1) and the symbol $\mathfrak{g}$ has a "small" norm. These properties are proved in the next lemma.

Theorem 7.2 Let $d=2$. The symbols $\mathfrak{f}, \mathfrak{g}$ defined above, satisfy the following properties:

(i) $\mathfrak{f}, \mathfrak{g} \in \mathbf{S}_{-2}(L)$ and $\left.\left|\mathfrak{f} \mathbf{|}_{l, s}^{(-2)}+\right| \mathfrak{g}\right|_{l, s} ^{(-2)} \leq C_{l, s}\left(|V|_{p, 0}^{(0)}\right)^{2}, p=p(l, s)$;

(ii) For some $z \in \mathbf{T}_{-2}(L)$ one has

$$
\begin{aligned}
\hat{\mathfrak{f}}(\boldsymbol{\chi}, \boldsymbol{\xi}) & =\hat{z}(\boldsymbol{\chi},\langle\boldsymbol{\xi}, \mathbf{n}(\boldsymbol{\chi})\rangle), \boldsymbol{\chi} \neq \mathbf{0}, \\
\boldsymbol{\|} z \boldsymbol{\|}_{l, s}^{(-2)} & \leq C_{l, s}\left(|\boldsymbol{|} V|_{p, 0}^{(0)}\right)^{2}, p=p(l, s) .
\end{aligned}
$$

(iii) Let the conditions (7.3) be fulfilled. Then

$$
\left\|\mathrm{Op}\left(\mathfrak{g}_{\nu}^{b}\right) \mathcal{P}\left(\hat{\Lambda}_{\nu}\right)\right\| \leq C_{p}\left(\mid V \mathbf{I}_{p, 0}^{(0)}\right)^{2} r^{4} \rho^{-2}, \hat{\Lambda}_{\nu}=\hat{\Lambda}_{\boldsymbol{\nu}}\left(\rho-L_{1}\right)
$$

with some integer $p$, uniformly in $\boldsymbol{\nu} \in \widetilde{\Theta}_{r}$.

The constants $C$ in the above inequalities are independent of $V$ and $\rho, L, r$.

Proof. Let us prove (i) first. By Lemma 6.3 we have $\left|V_{2}+t_{2}\right|_{l, s}^{(-2)} \leq C_{l, s}\left(|V|_{p, 0}^{(0)}\right)^{2}$ with some $p=p(l, s)$. By (4.14) this guarantees the same estimate for $V_{2}^{o}$ and $t_{2}^{o}$. Consequently, part (i) will be proved if we establish this estimate for $\mathfrak{f}$ only. The required bound follows from (7.1) in view of (4.14).

Proof of (ii). Use (7.2) with $\boldsymbol{\mu}=\boldsymbol{\eta}=\boldsymbol{\nu}$, and a similar formula for $\mathfrak{b}_{\boldsymbol{\nu}, \boldsymbol{\nu}}$. Recalling that

$$
\begin{gathered}
\varphi_{\boldsymbol{\theta}}(\boldsymbol{\xi} ; L)=1-\Upsilon\left(L^{-1}\left(\langle\mathbf{n}(\boldsymbol{\theta}), \boldsymbol{\xi}\rangle+2^{-1}|\boldsymbol{\theta}|\right)\right), \\
\tau_{\boldsymbol{\theta}}(\boldsymbol{\xi})=2\langle\boldsymbol{\theta}, \boldsymbol{\xi}+\boldsymbol{\theta} / 2\rangle,
\end{gathered}
$$

by Definitions (4.2) and (6.10), we conclude that $\hat{\mathfrak{f}}_{\nu}=\hat{\mathfrak{a}}_{\nu, \nu}+\hat{\mathfrak{b}}_{\nu, \nu}$ has the form (5.1). This means that $\hat{f}$ also has this property, that is $\hat{f}$ satisfies (7.8) with some function $z$. Moreover, in view of (5.2) and part (i), the function $z$ belongs to $\mathbf{T}_{-2}$ and satisfies the bound (7.9). One can write an explicit formula for the function $z$, but it is too cumbersome and is therefore omitted.

Proof of (iii). The estimate (7.10) follows from Definition (7.7) by virtue of (7.4) and Proposition 3.1. 


\subsection{Operator $A_{1}$}

Now we use the established results to study the operator $A_{1}$ from Section 6 with $M=2$. According to $(6.9)$

$$
\left\{\begin{array}{l}
A_{1}=A^{o}+V^{\mathrm{b}}+\left(V_{2}^{\mathrm{b}}+T_{2}^{\mathrm{b}}\right)+X_{2}^{\uparrow}+R_{3} \\
A^{o}=H_{0}+V_{2}^{o}+T_{2}^{o}
\end{array}\right.
$$

Recall that $\hat{V}(\mathbf{0})=0$, so that the term $V_{1}^{o}$ drops out. The remainders $X_{2}^{\uparrow}$ and $R_{3}$ satisfy the bounds

$$
\left\|R_{3}\right\| \leq C L^{-4}, \quad\left\|X_{2}^{\uparrow}\right\| \leq C_{p} r^{-p}, \forall p>0
$$

Indeed, the estimate for $R_{3}$ follows from (6.18) used with $\alpha=0$ and $M=2$. Furthermore, the estimate for $X_{2}^{\uparrow}$ is a consequence of (6.19), (4.10) and Remark 3.2.

In this section we establish a suitable asymptotic formula for the density of states of the operator $A^{o}+V^{b}+V_{2}^{b}+T_{2}^{b}$ with the help of Theorem 5.4. Let us verify first that the symbol $a^{o}$ has the required form.

Lemma 7.3 Let $d \geq 2$. The symbol $a^{o}$ has the form (5.21) with

$$
f^{(\boldsymbol{\nu})}(\eta)=-\frac{1}{\mathrm{~d}(\Gamma)} \sum_{\substack{0 \neq l \in \mathbb{Z}, l \boldsymbol{\nu} \in \Theta_{r}}} \frac{|\hat{V}(l \boldsymbol{\nu})|^{2}}{2 l|\boldsymbol{\nu}|(\eta+l|\boldsymbol{\nu}| / 2)}\left[1-\Upsilon^{2}\left((\eta+l|\boldsymbol{\nu}| / 2) L^{-1}\right)\right], \boldsymbol{\nu} \in \tilde{\Theta}_{r}
$$

The function $f=\sum_{\boldsymbol{\nu} \in \widetilde{\Theta}_{r}} f^{(\boldsymbol{\nu})}$ in (5.21) belongs to $\mathbf{S}_{-2}(L)$ uniformly in $V$. Moreover, under the condition $1 \leq r \leq L$ one has

$$
\sup _{|\eta| \geq 4 L}|\eta|^{2}\left|f^{(\boldsymbol{\nu})}(\eta)\right| \leq \varkappa_{\boldsymbol{\nu}}, \quad \varkappa=\sum_{\boldsymbol{\nu} \in \widetilde{\Theta}_{r}} \varkappa_{\boldsymbol{\nu}} \leq \frac{1}{\mathrm{~d}(\Gamma)}\|V\|_{\mathrm{L}^{2}}^{2} .
$$

Proof. We need to show that the symbol $V_{2}^{o}+t_{2}^{o}$ has the form $f(\boldsymbol{\xi})$ as specified in (5.21). To this end rewrite (6.17), replacing the sum over all $\boldsymbol{\theta} \in \Theta_{r}$ by the double sum

$$
\sum_{\nu \in \widetilde{\Theta}_{r}} \sum_{\boldsymbol{\theta} \in \Gamma_{\nu}^{\dagger} \cap \Theta_{r}}
$$

Now, denoting $\boldsymbol{\theta}=l \boldsymbol{\nu}, 0 \neq l \in \mathbb{Z}$ and $\eta=\langle\boldsymbol{\xi}, \mathbf{n}(\boldsymbol{\nu})\rangle$ we can write for each $\boldsymbol{\theta} \in \Gamma_{\boldsymbol{\nu}}^{\dagger}$ that

$$
\zeta_{\boldsymbol{\theta}}(\boldsymbol{\xi} ; L)=\Upsilon\left((\eta+l|\boldsymbol{\nu}| / 2) L^{-1}\right), \quad \tau_{\boldsymbol{\theta}}(\boldsymbol{\xi})=2 l|\boldsymbol{\nu}|(\eta+l|\boldsymbol{\nu}| / 2)
$$

Now it is clear that $f=V_{2}^{o}+t_{2}^{o}$ has the form (5.21) with $f^{(\boldsymbol{\nu})}$ as in (7.13). Observe also that according to Lemma 6.3 (ii), the function $f$ belongs to $\mathbf{S}_{-2}$ uniformly 
in $V$. In order to establish (7.14) observe that for $r \leq L$ and $|\eta| \geq 4 L$ the function $\Upsilon$ in the Definition (7.13) vanishes (see Definition (4.1)), and hence

$$
\begin{aligned}
f^{(\boldsymbol{\nu})}(\eta) & =-\frac{1}{\mathrm{~d}(\Gamma)|\boldsymbol{\nu}|} \sum_{\substack{0<l \in \mathbb{Z}, l \boldsymbol{\nu} \in \Theta_{r}}}|\hat{V}(l \boldsymbol{\nu})|^{2}\left[\frac{1}{2 l(\eta+l|\boldsymbol{\nu}| / 2)}-\frac{1}{2 l(\eta-l|\boldsymbol{\nu}| / 2)}\right] \\
& =\frac{1}{2 \mathrm{~d}(\Gamma)} \sum_{\substack{0<l \in \mathbb{Z}, l \boldsymbol{\nu} \in \Theta_{r}}}|\hat{V}(l \boldsymbol{\nu})|^{2} \frac{1}{\eta^{2}-l^{2}|\boldsymbol{\nu}|^{2} / 4}
\end{aligned}
$$

Remembering again that $|\eta| \geq 4 L \geq 4 r \geq 4|l||\boldsymbol{\nu}|$, we conclude that for $|\eta| \geq 4 L$

$$
\left|f^{(\boldsymbol{\nu})}(\eta)\right| \leq \varkappa_{\boldsymbol{\nu}} \eta^{-2}, \quad \varkappa_{\boldsymbol{\nu}}=\frac{1}{\mathrm{~d}(\Gamma)} \sum_{l}|\hat{V}(l \boldsymbol{\nu})|^{2},
$$

and hence

$$
\varkappa=\sum_{\boldsymbol{\nu} \in \tilde{\Theta}_{r}} \varkappa_{\boldsymbol{\nu}} \leq \frac{1}{\mathrm{~d}(\Gamma)} \sum_{\boldsymbol{\theta} \neq 0}|\hat{V}(\boldsymbol{\theta})|^{2}=\frac{1}{\mathrm{~d}(\Gamma)}\|V\|_{\mathrm{L}^{2}}^{2},
$$

as required.

We need to specify the operators which will play the role of $B$ and $Q$ in Theorem 5.4. Let $\mathfrak{f}$ and $\mathfrak{g}$ be as defined in (7.7). We use Theorem 5.4 with $B=$ $\operatorname{Op}(b), Q=\operatorname{Op}(q)$ where

$$
b=V+\mathfrak{f}, q=\mathfrak{g} .
$$

Let us establish a useful estimate for the operator $Q$ stating the result in a form of a lemma for the reference convenience:

Lemma 7.4 Let $d=2$. Under conditions (7.3) one has

$$
\delta=\max _{\boldsymbol{\nu} \in \widetilde{\Theta}_{r}}\left\|Q_{\nu}^{b} \mathcal{P}\left(\hat{\Lambda}_{\nu}\right)\right\| \leq C r^{4} \rho^{-2}, \hat{\Lambda}_{\nu}=\hat{\Lambda}_{\nu}\left(\rho-L_{1}\right)
$$

with a constant uniform in $V$.

Proof. The sought result immediately follows from the Definition (7.15) and the bound (7.10).

Theorem 7.5 Let $d=2$ and let $\varkappa$ and $\delta$ be as defined in (7.14) and (7.16). Let $\lambda \in\left[\rho^{2}-\lambda_{0}, \rho^{2}+\lambda_{0}\right]$ with some $\lambda_{0} \geq 1$, and let $\rho^{2} \geq 20 \lambda_{0}$. Assume that (7.3) and (5.24) are satisfied. Then there exists a constant $L_{0}=L_{0}(V)$ such that under the condition (5.25) one has

$$
\left|D\left(\lambda ; A^{o}+V^{b}+V_{2}^{b}+T_{2}^{b}\right)-\frac{1}{(2 \pi)^{2}} \operatorname{vol} \mathcal{E}\left(\lambda ; a^{o}\right)\right| \leq C r^{6} \rho^{-3} L .
$$

The constants $L_{0}, C$ are uniform in $V$ and do not depend on $\lambda, \rho, L, r$. 
Proof. Note first of all that in view of (7.3) the conditions (5.23) are satisfied. Moreover, by the third estimate in (7.3), $\delta \leq C L^{-2}$ and for sufficiently large $L \geq L_{0}$ the condition $\rho^{2} \geq 20 \lambda_{0}$ will ensure that $\rho^{2} \geq 16 \lambda_{1}$ with the number $\lambda_{1}$ defined in (5.22).

Let us check now that further conditions of Theorem 5.4 are fulfilled. Let $B=\mathrm{Op}(b)$ and $Q=\mathrm{Op}(q)$ with the symbols $b, q$ defined in (7.15). From Theorem 7.2(i) we know that $q \in \mathbf{P}_{0}(L)$, and that the symbol $b$ can be represented in the form (5.1) with some $z \in \mathbf{T}_{0}(L)$. Besides, according to Lemma 7.3 the symbol $a^{o}=h_{0}+V_{2}^{o}+t_{2}^{o}$ can be represented in the form (5.21), and the property (5.20) is satisfied with $\beta=2$ and $\varkappa_{\nu}$ specified in (7.14). Remembering the bound (7.16) and applying Theorem 5.4, we obtain that

$\left|D\left(\lambda ; A^{o}+V^{b}+V_{2}^{b}+T_{2}^{b}\right)-\frac{1}{(2 \pi)^{2}} \operatorname{vol} \mathcal{E}\left(\lambda ; a^{o}\right)\right| \leq C r^{2} \rho^{-1} L\left(r^{4} \rho^{-2}+r^{4} \rho^{-2}\right)+C r^{2} \rho^{-3} L$,

which leads to the stated estimate.

\section{Density of states for operator $A^{o}$. Proof of Theorem 2.3}

\subsection{Operator $A^{o}$}

The last step of the proof of Theorem 2.3 is the asymptotics of the quantity $\operatorname{vol} \varepsilon\left(\lambda ; a^{o}\right)$. Recall that $a^{o}(\boldsymbol{\xi})=|\boldsymbol{\xi}|^{2}+f(\boldsymbol{\xi}), \quad f=V_{2}^{o}+t_{2}^{o}$. Now we do not need the formula (7.13) for $f$, but apply the initial formula (6.17):

$$
f(\boldsymbol{\xi})=-\frac{1}{\mathrm{~d}(\Gamma)} \sum_{\boldsymbol{\theta} \in \Theta_{r}} \frac{|\hat{V}(\boldsymbol{\theta})|^{2}}{\tau_{\boldsymbol{\theta}}(\boldsymbol{\xi})}\left(1-\zeta_{\boldsymbol{\theta}}^{2}(\boldsymbol{\xi} ; L)\right) .
$$

Recall that $\tau_{\boldsymbol{\theta}}$ and $\zeta_{\boldsymbol{\theta}}$ are defined in (6.10) and (4.2) respectively. The calculations in this section are done for the case of a general dimension $d \geq 2$.

Our aim is to prove

Theorem 8.1 Let $a^{o}$ be as defined above. Suppose that $2 L \leq \rho^{\epsilon}$ with some $\epsilon \in(0,1)$. Then for any $l>d / 2$ one has

$$
\begin{aligned}
& \left.\left|\operatorname{vol} \varepsilon\left(\rho^{2} ; a^{o}\right)-\mathrm{w}_{d} \rho^{d}-\frac{d(d-2) \mathrm{w}_{d}}{8 \mathrm{~d}(\Gamma)} \rho^{d-4} \int_{\mathcal{O}}\right| V(\mathbf{x})\right|^{2} d \mathbf{x} \mid \\
& \leq C_{l} \rho^{d-4}\left[\rho^{\epsilon-1}+\rho^{4 \epsilon-3} \ln \rho+r^{-2 l+d}+L^{-4}+\rho L^{-5}\right] .
\end{aligned}
$$

The constant $C_{l}$ is uniform in $V$.

To begin with, we need to find the formula describing the level surface $a^{o}(\boldsymbol{\xi})=$ $\rho^{2}$. Denote

$$
\boldsymbol{\xi}=t \boldsymbol{\omega}, \quad t=|\boldsymbol{\xi}|,|\boldsymbol{\omega}|=1
$$


Rewrite the equation $a^{o}(\boldsymbol{\xi})=\rho^{2}$ :

$$
t^{2}+f(t \omega)=\rho^{2}
$$

and solve it for $t$. Since $f \in \mathbf{S}_{-2}(L)$ (see Lemma 7.3), one can write $\left|\nabla_{\boldsymbol{\xi}} f(\boldsymbol{\xi})\right| \leq$ $C L^{-3}$, so that the solution of this equation is

$$
t=t(\rho, \boldsymbol{\omega})=\rho-\frac{f(\rho \boldsymbol{\omega})}{2 \rho}+O\left(\rho^{-3} L^{-4}\right)+O\left(\rho^{-2} L^{-5}\right) .
$$

Here and further on all remainder estimates are uniform in $V$. Therefore

$$
\begin{aligned}
\operatorname{vol} \mathcal{E}\left(\rho^{2} ; a^{o}\right) & =\int_{\mathbb{S}^{d-1}} \int_{0}^{t(\rho, \boldsymbol{\omega})} \tau^{d-1} d \tau d \boldsymbol{\omega}=\frac{1}{d} \int_{\mathbb{S}^{d-1}}(t(\rho, \boldsymbol{\omega}))^{d} d \boldsymbol{\omega} \\
& =\frac{1}{d} \int_{\mathbb{S}^{d-1}}\left[\rho^{d}-d \rho^{d-2} \frac{f}{2}\right] d \boldsymbol{\omega}+O\left(\rho^{d-4} L^{-4}\right)+O\left(\rho^{d-3} L^{-5}\right) \\
& =\mathrm{w}_{d} \rho^{d}-\rho^{d-2} \frac{1}{2} \int_{\mathbb{S}^{d-1}} f(\rho \boldsymbol{\omega}) d \boldsymbol{\omega}+O\left(\rho^{d-4} L^{-4}\right)+O\left(\rho^{d-3} L^{-5}\right) .
\end{aligned}
$$

We concentrate on the second term:

$$
M(\rho)=\frac{1}{2} \int_{\mathbb{S}^{d-1}} f(\rho \boldsymbol{\omega}) d \boldsymbol{\omega}
$$

Let us rewrite the function $f$ in a more manageable form:

$$
f(\boldsymbol{\xi})=-\frac{1}{\mathrm{~d}(\Gamma)} \sum_{\boldsymbol{\theta} \in \Theta_{r}} \frac{\mid \hat{V}(\boldsymbol{\theta}))\left.\right|^{2}}{2|\boldsymbol{\theta}|\langle\mathbf{n}(\boldsymbol{\theta}), \boldsymbol{\xi}\rangle+|\boldsymbol{\theta}|^{2}} g(\langle\mathbf{n}(\boldsymbol{\theta}), \boldsymbol{\xi}\rangle+|\boldsymbol{\theta}| / 2),
$$

where

$$
g(\eta)=1-\Upsilon^{2}\left(\eta L^{-1}\right)
$$

The value of $f(\boldsymbol{\xi})$ will not change if we replace $\boldsymbol{\theta}$ by $-\boldsymbol{\theta}$ in the above sum. Remembering that the function $\Upsilon$ is even ( see (4.1)) and adding up both sums we conclude that

$$
\begin{gathered}
f(\boldsymbol{\xi})=-\frac{1}{4 \mathrm{~d}(\Gamma)} \sum_{\boldsymbol{\theta} \in \Theta_{r}} \frac{\mid \hat{V}(\boldsymbol{\theta}))\left.\right|^{2}}{|\boldsymbol{\theta}|} z(\langle\mathbf{n}(\boldsymbol{\theta}), \boldsymbol{\xi}\rangle ;|\boldsymbol{\theta}| / 2), \\
z\left(\eta ; \eta_{0}\right)=\frac{g\left(\eta+\eta_{0}\right)}{\eta+\eta_{0}}-\frac{g\left(\eta-\eta_{0}\right)}{\eta-\eta_{0}} .
\end{gathered}
$$

We begin the calculation of the integral $M$ in (8.1) with finding the asymptotics of the integral

$$
Z\left(\rho ; \eta_{0}\right)=\int_{\mathbb{S}^{d-1}} z\left(\rho\left\langle\boldsymbol{\omega}, \mathbf{e}_{1}\right\rangle ; \eta_{0}\right) d \boldsymbol{\omega},
$$

as $\rho \rightarrow \infty$. 
Lemma 8.2 Let the functions $g$ and $z$ be as defined above, and let $0 \leq \eta_{0} \leq L$, $2 L \leq \rho^{\epsilon}$ for some $\epsilon \in(0,1)$. Then

$$
Z\left(\rho ; \eta_{0}\right)=2 d(d-2) \mathrm{w}_{d} \eta_{0} \rho^{-2}+\eta_{0} O\left(\rho^{\epsilon-3}\right)+O\left(\rho^{4 \epsilon-5} \ln \rho\right)+O\left(\eta_{0}^{3} \rho^{-4}\right) .
$$

Proof. The integral at hand can be rewritten as follows:

$$
\begin{aligned}
Z\left(\rho ; \eta_{0}\right) & =\int_{\mathbb{S}^{d-2}} d \boldsymbol{\phi} \int_{0}^{\pi} z\left(\rho \cos \beta ; \eta_{0}\right) \sin ^{d-2} \beta d \beta=\omega_{d-2} \int_{-1}^{1} z\left(\rho t, \eta_{0}\right)\left(1-t^{2}\right)^{\varkappa} d t \\
& =\omega_{d-2} \rho^{-1} \int_{-\rho}^{\rho} z\left(t, \eta_{0}\right)\left(1-t^{2} \rho^{-2}\right)^{\varkappa} d t, \quad \varkappa=\frac{d-3}{2} .
\end{aligned}
$$

Here $\omega_{d-2}$ is defined in (5.11). Denote

$$
S_{1}(\rho)=\int_{-\rho}^{\rho} \frac{g\left(t+\eta_{0}\right)}{t+\eta_{0}}\left(1-t^{2} \rho^{-2}\right)^{\varkappa} d t, \quad S_{2}(\rho)=\int_{-\rho}^{\rho} \frac{g\left(t-\eta_{0}\right)}{t-\eta_{0}}\left(1-t^{2} \rho^{-2}\right)^{\varkappa} d t,
$$

so that $Z=\rho^{-1} \omega_{d-2}\left(S_{1}-S_{2}\right)$.

Step 1. Each of these two integrals is split into the sum of two integrals in the following way. Represent the integrals $S_{1}, S_{2}$ in the form

$$
\begin{aligned}
S_{1} & =S_{11}+S_{12}, \quad S_{2}=S_{21}+S_{22}, \\
S_{11} & =\int_{-\rho^{\epsilon}-\eta_{0}}^{\rho^{\epsilon}-\eta_{0}} \frac{g\left(t+\eta_{0}\right)}{t+\eta_{0}}\left(1-t^{2} \rho^{-2}\right)^{\varkappa} d t, \\
S_{12} & = \\
S_{21} & =\int_{-\rho^{\epsilon}+\eta_{0}} \frac{g\left(t+\eta_{0}\right)}{t+\eta_{0}}\left(1-t^{2} \rho^{-2}\right)^{\varkappa} d t, \\
S_{22} & = \\
\rho_{(-\rho, \rho) \backslash\left(-\eta_{0}^{\epsilon}+\eta_{0}, \rho^{\epsilon}+\eta_{0}\right)} & \frac{g\left(t-\eta_{0}, \rho^{\epsilon}-\eta_{0}\right)}{\left.t-\eta_{0}\right)}\left(1-t_{0}^{2} \rho^{-2}\right)^{\varkappa} d t,
\end{aligned}
$$

For the integrals $S_{11}$ and $S_{21}$ we use the decomposition

$$
\left(1-t^{2} \rho^{-2}\right)^{\varkappa}=1-\varkappa t^{2} \rho^{-2}+O\left(\rho^{4(\epsilon-1)}\right),|t| \leq C \rho^{\epsilon},
$$

Recalling Definitions (8.3), (4.1) one can write

$$
S_{11}(\rho)=\tilde{S}_{11}+O\left(\rho^{4(\epsilon-1)} \ln \rho\right), \quad S_{21}(\rho)=\tilde{S}_{21}+O\left(\rho^{4(\epsilon-1)} \ln \rho\right),
$$

with

$$
\begin{gathered}
\tilde{S}_{11}(\rho)=\int_{-\rho^{\epsilon}-\eta_{0}}^{\rho^{\epsilon}-\eta_{0}} \frac{g\left(t+\eta_{0}\right)}{t+\eta_{0}}\left(1-\varkappa t^{2} \rho^{-2}\right) d t=\int_{-\rho^{\epsilon}}^{\rho^{\epsilon}} \frac{g(t)}{t}\left[1-\varkappa\left(t-\eta_{0}\right)^{2} \rho^{-2}\right] d t \\
\tilde{S}_{21}(\rho)=\int_{-\rho^{\epsilon}}^{\rho^{\epsilon}} \frac{g(t)}{t}\left[1-\varkappa\left(t+\eta_{0}\right)^{2} \rho^{-2}\right] d t .
\end{gathered}
$$


Consequently,

$$
\begin{aligned}
S_{11}-S_{21} & =\tilde{S}_{11}-\tilde{S}_{21}+O\left(\rho^{4(\epsilon-1)} \ln \rho\right) \\
& =4 \varkappa \eta_{0} \rho^{-2} \int_{-\rho^{\epsilon}}^{\rho^{\epsilon}} g(t) d t+O\left(\rho^{4(\epsilon-1)} \ln \rho\right) \\
& =O\left(\eta_{0} \rho^{\epsilon-2}\right)+O\left(\rho^{4(\epsilon-1)} \ln \rho\right) .
\end{aligned}
$$

Step 2. Let us now concentrate on the remaining integrals $S_{12}, S_{22}$. Introduce the following contours (paths):

$$
\begin{gathered}
\ell_{12}=[-\rho, \rho] \backslash\left(-\rho^{\epsilon}-\eta_{0}, \rho^{\epsilon}-\eta_{0}\right), \ell_{22}=[-\rho, \rho] \backslash\left(-\rho^{\epsilon}+\eta_{0}, \rho^{\epsilon}+\eta_{0}\right), \\
\ell_{11}=\left\{z \in \mathbb{C}: \operatorname{Im} z \geq 0,\left|z+\eta_{0}\right|=\rho^{\epsilon}\right\}, \quad \ell_{21}=\left\{z \in \mathbb{C}: \operatorname{Im} z \geq 0,\left|z-\eta_{0}\right|=\rho^{\epsilon}\right\}, \\
\ell_{2}=\{z \in \mathbb{C}: \operatorname{Im} z \geq 0,|z|=\rho\} .
\end{gathered}
$$

The paths $\ell_{12}, \ell_{22}$ consist of two segments each. Define also

$$
\ell_{1}=\left\{z \in \mathbb{C}: \operatorname{Im} z \geq 0,|z|=\rho^{\epsilon}\right\},
$$

so that $\ell_{11}, \ell_{21}$ can be rewritten as

$$
\ell_{11}=\left\{z \in \mathbb{C}: z+\eta_{0} \in \ell_{1}\right\}, \quad \ell_{21}=\left\{z \in \mathbb{C}: z-\eta_{0} \in \ell_{1}\right\} .
$$

By Definition (8.3) and because of the conditions $2 L \leq \rho^{\epsilon}, \eta_{0} \leq L$, we have $g\left(t \pm \eta_{0}\right)=1$ for all $|t| \geq \rho^{\epsilon}$, and hence

$$
S_{12}=\int_{\ell_{12}} \frac{1}{t+\eta_{0}}\left(1-t^{2} \rho^{-2}\right)^{\varkappa} d t, \quad S_{22}=\int_{\ell_{22}} \frac{1}{t-\eta_{0}}\left(1-t^{2} \rho^{-2}\right)^{\varkappa} d t .
$$

In view of the analyticity of the integrands away from $\pm t_{0}$ and $\pm \rho$, these integrals can be rewritten as follows:

$$
S_{12}=\left(\int_{\ell_{11}}-\int_{\ell_{2}}\right) \frac{1}{z+\eta_{0}}\left(1-z^{2} \rho^{-2}\right)^{\varkappa} d z, \quad S_{22}=\left(\int_{\ell_{21}}-\int_{\ell_{2}}\right) \frac{1}{z-\eta_{0}}\left(1-z^{2} \rho^{-2}\right)^{\varkappa} d z .
$$

The direction of integration is counter-clockwise. Let us show first that the integrals over $\ell_{11}$ and $\ell_{21}$ give a lower order contribution. Using (8.4), as in Step 1 write the difference of these integrals in the form:

$$
4 \varkappa \eta_{0} \rho^{-2} \int_{\ell_{1}} d z+O\left(\rho^{4(\epsilon-1)} \ln \rho\right)=\eta_{0} O\left(\rho^{\epsilon-2}\right)+O\left(\rho^{4(\epsilon-1)} \ln \rho\right) .
$$

Consider now the difference of the integrals over the large semi-circle $\ell_{2}$ :

$$
\begin{aligned}
-\int_{\ell_{2}}\left[\frac{1}{z+\eta_{0}}-\frac{1}{z-\eta_{0}}\right]\left(1-z^{2} \rho^{-2}\right)^{\varkappa} d z & =2 \eta_{0} \int_{\ell_{2}} \frac{1}{z^{2}-\eta_{0}^{2}}\left(1-z^{2} \rho^{-2}\right)^{\varkappa} d z \\
& =2 \eta_{0} \int_{\ell_{2}} \frac{1}{z^{2}}\left(1-z^{2} \rho^{-2}\right)^{\varkappa} d z+O\left(\eta_{0}^{3} \rho^{-3}\right) .
\end{aligned}
$$


After rewriting the integral over $\ell_{2}$ with substitution $z=\rho e^{i \phi}, \phi \in[0, \pi]$, and collecting all the pieces together we obtain:

$$
\begin{gathered}
S_{21}-S_{22}=2 \eta_{0} \rho^{-1} J+\eta_{0} O\left(\rho^{\epsilon-2}\right)+O\left(\rho^{4(\epsilon-1)} \ln \rho\right)+O\left(\eta_{0}^{3} \rho^{-3}\right), \\
J=i \int_{0}^{\pi} e^{-i \phi}\left(1-e^{2 i \phi}\right)^{\varkappa} d \phi .
\end{gathered}
$$

Adding to this the estimate for $S_{11}-S_{21}$ obtained on Step I, recalling that $Z=$ $\omega_{d-2} \rho^{-1}\left(S_{1}-S_{2}\right)$, and using the formula (9.1) derived in the Appendix, we arrive at the expected formula.

Let us now calculate $M(\rho)$, see (8.2):

Lemma 8.3 Let $r \leq 2 L \leq \rho^{\epsilon}$ for some $\epsilon \in(0,1)$. Then for any $l>d / 2$

$$
\left|M(\rho)+\frac{d(d-2) \mathrm{w}_{d}}{8 \rho^{2} \mathrm{~d}(\Gamma)}\|V\|_{\mathrm{L}^{2}(\mathcal{O})}^{2}\right| \leq C_{l}\left(\rho^{-2} r^{-2 l+d}+\rho^{\epsilon-3}+\rho^{4 \epsilon-5} \ln \rho\right)
$$

with a constant $C_{l}$ uniform in $V$.

Proof. It follows from definition of $Z\left(\rho ; \eta_{0}\right)$ that

$$
M=-\frac{1}{8 \mathrm{~d}(\Gamma)} \sum_{\boldsymbol{\theta} \in \Theta_{r}} \frac{|\hat{V}(\boldsymbol{\theta})|^{2}}{|\boldsymbol{\theta}|} Z(\rho ;|\boldsymbol{\theta}| / 2) .
$$

According to the Lemma 8.2 and Parceval's identity the leading term of the r.h.s. is given by

$$
-\frac{d(d-2) \mathrm{w}_{d}}{8 \rho^{2} \mathrm{~d}(\Gamma)} \sum_{\boldsymbol{\theta} \in \Theta_{r}}|\hat{V}(\boldsymbol{\theta})|^{2}=-\frac{d(d-2) \mathrm{w}_{d}}{8 \rho^{2} \mathrm{~d}(\Gamma)}\|V\|_{L^{2}(\mathcal{O})}^{2}+O\left(\rho^{-2}\right) \sum_{\boldsymbol{\theta} \in \Xi_{r}}|\hat{V}(\boldsymbol{\theta})|^{2},
$$

see (4.5) for definition of $\Xi_{r}$. The error term does not exceed, up to a multiplicative constant independent of $\rho$ and $V$,

$$
\left(\rho^{\epsilon-3}+\rho^{4 \epsilon-5} \ln \rho\right)\|V\|_{\mathbf{L}^{2}(\mathcal{O})}^{2}+\rho^{-4}\|\Delta V\|_{\mathbf{L}^{2}(\mathcal{O})}^{2} .
$$

In view of (2.3), for any $l>d / 2$ one has

$$
\sum_{\boldsymbol{\theta} \in \Xi_{r}}|\hat{V}(\boldsymbol{\theta})|^{2} \leq\left.\left|V \mathbf{l}_{l, 0}^{(0)} \sum_{|\boldsymbol{\theta}| \geq r}\right| \boldsymbol{\theta}\right|^{-2 l} \leq C_{l} \mid V \mathbf{|}_{l, 0}^{(0)} r^{-2 l+d} .
$$

This leads to the proclaimed formula.

Proof of Theorem 8.1. The required asymptotics immediately follows from Lemma 8.3 and formula (8.1). 


\subsection{Completion of the proof of Theorem 2.3}

In contrast to the previous subsection, where we could allow any dimension $d \geq 2$, now we restrict ourselves to $d=2$ only.

Recall that the unitary operator $U=e^{i \Psi}$ constructed in Section 6 is $\Gamma$ periodic. Consequently, by Proposition 2.1(ii), we have $D(\lambda ; H)=D\left(\lambda ; A_{1}\right)$, and hence it remains to establish the sought asymptotics for the operator $A_{1}$ only. To this end we shall use the formula (7.11). By monotonicity of the density of states (see Proposition 2.1(i)) the formulae (7.12) and (7.11) give the estimates

$$
\begin{aligned}
D\left(\rho^{2}-C L^{-4}-C r^{-p} ; A^{o}+B^{b}\right. & \left.+B_{2}^{b}+T_{2}^{b}\right) \leq D\left(\rho^{2} ; A_{1}\right) \\
& \leq D\left(\rho^{2}+C L^{-4}+C r^{-p} ; A^{o}+B^{b}+B_{2}^{b}+T_{2}^{b}\right) .
\end{aligned}
$$

In order to apply Theorem 7.5, assume that $L=L_{1}=\rho^{\epsilon} / 2, r=\rho^{\beta}$ with some $\epsilon \in(0,1)$ and $\beta \in(0, \min \{\epsilon,(1-\epsilon) / 2\})$, so that the conditions (7.3), (5.23), (5.25) are satisfied for all $\rho \geq \rho_{0}$ with a sufficiently large $\rho_{0}=\rho_{0}(V)$ which is uniform in $V$. According to Theorems 7.5 and 8.1 we have

$$
\begin{aligned}
& \left|D\left(\rho^{2} ; A_{1}\right)-\frac{\mathrm{w}_{2}}{(2 \pi)^{2}} \rho^{2}\right| \leq C_{l} \rho^{-2}\left[\rho^{\epsilon-1}+\rho^{4 \epsilon-3} \ln \rho\right. \\
& \left.\quad+r^{-2 l+d}+L^{-4}+\rho L^{-5}+r^{6} \rho^{-1} L\right]+C^{\prime} L^{-4}+C_{p}^{\prime \prime} r^{-p}, \forall l>d / 2, \forall p>0 .
\end{aligned}
$$

Substitute $L=\rho^{\epsilon} / 2$ and $r=\rho^{\beta}$ :

$$
C \rho^{-2}\left[r^{6 \beta} \rho^{\epsilon-1}+\rho^{4 \epsilon-3} \ln \rho+\rho^{2-4 \epsilon}\right]+C_{p}^{\prime} \rho^{-p \beta}, \forall p>0 .
$$

Optimizing in $\epsilon$ we get $\epsilon=3 / 5$. Choose an arbitrarily small $\beta$ and a suitably large $p$.

\section{Appendix}

Our aim is to find the value of the integral

$$
J=i \int_{0}^{\pi} e^{-i \phi}\left(1-e^{2 i \phi}\right)^{\varkappa} d \phi, \varkappa=\frac{d-3}{2},
$$

featuring in the proof of Lemma 8.2.

Lemma 9.1 The integral $J$ is given by

$$
J=d(d-2) \frac{\mathrm{w}_{d}}{\omega_{d-2}}=\sqrt{\pi} \frac{d(d-2)}{d-1} \frac{\Gamma\left(\frac{d+1}{2}\right)}{\Gamma\left(\frac{d+2}{2}\right)} .
$$

Proof. Recall that (see (1.3), (5.11))

$$
\mathrm{w}_{d}=\frac{\pi^{\frac{d}{2}}}{\Gamma\left(\frac{d+2}{2}\right)}, \quad \omega_{d-2}=(d-1) \mathrm{w}_{d-1}=\frac{(d-1) \pi^{\frac{d-1}{2}}}{\Gamma\left(\frac{d+1}{2}\right)},
$$

so that we need to prove only that $J$ coincides with the r.h.s. of (9.1). 
Define

$$
J(t)=i \int_{0}^{\pi} e^{-i \phi}\left(1-t e^{2 i \phi}\right)^{\varkappa} d \phi,|t|<1 .
$$

Expanding the integrand in the absolutely convergent series, we find

$$
J=\lim _{t \uparrow 1} J(t)=\lim _{t \uparrow 1} i \int_{0}^{\pi} \sum_{n=0}^{\infty} t^{n} e^{(2 n-1) i \phi}(-1)^{n}\left(\begin{array}{l}
\varkappa \\
n
\end{array}\right) d \phi=-2 \sum_{n=0}^{\infty}(-1)^{n} \frac{1}{2 n-1}\left(\begin{array}{l}
\varkappa \\
n
\end{array}\right) .
$$

For $d=2$ we have $\varkappa=-1 / 2$ and we can use the formula

$$
\left(\begin{array}{c}
-1 / 2 \\
n
\end{array}\right)=\left(\begin{array}{c}
1 / 2 \\
n
\end{array}\right)(1-2 n)
$$

so that

$$
J=2 \sum_{n=0}^{\infty}(-1)^{n}\left(\begin{array}{c}
1 / 2 \\
n
\end{array}\right)=2(1-1)^{1 / 2}=0 .
$$

For $d \geq 3$ use [3], formula 1.4(2), which implies that the r.h.s. of (9.2) coincides with

$$
-\frac{\Gamma\left(-\frac{1}{2}\right) \Gamma(\varkappa+1)}{\Gamma\left(\varkappa+\frac{1}{2}\right)}=2 \sqrt{\pi} \frac{\Gamma\left(\frac{d-1}{2}\right)}{\Gamma\left(\frac{d-2}{2}\right)}
$$

In view of the relations

$$
\Gamma\left(\frac{d+1}{2}\right)=\frac{d-1}{2} \Gamma\left(\frac{d-1}{2}\right), \Gamma\left(\frac{d+2}{2}\right)=\frac{d(d-2)}{4} \Gamma\left(\frac{d-2}{2}\right),
$$

this leads to $(9.1)$.

Acknowledgments. The author is grateful to L. Parnovski for discussions. The paper was completed during the author's stay at the Mittag-Leffler Institute in September 2002.

\section{References}

[1] M.S. Agranovich, Elliptic operators on closed manifolds, Itogi Nauki Tekh., Ser. Sovrem. Probl. Mat., Fundam. Napravleniya 63, 5-129 (1994); Engl. transl. in: Partial differential equations. VI, Encycl. Math. Sci. 63, 1-130 (1994).

[2] B.E.J. Dahlberg, E. Trubowitz, A remark on two-dimensional periodic potentials, Comment. Math. Helvetici 57, 130-134 (1982).

[3] A. Erdélyi, Higher transcendental functions, V. I, McGraw-Hill 1953. 
[4] J. Feldman, H. Knörrer, E. Trubowitz, The perturbatively stable spectrum of a periodic Schrödinger operator, Invent. Math. 100, 259-300 (1990).

[5] tor, Comment. Math. Helvetici 66, 557-579 (1991).

[6] B. Helffer, A. Mohamed, Asymptotics of the density of states for the Schrödinger operator with periodic electric potential, Duke Math. J. 92, 1-60 (1998).

[7] M. Hitrik, I. Polterovich, Regularized traces and Taylor expansions for the heat semigroup, J. London Math. Soc.II. Ser. 68, No. 2, 402-418 (2003).

[8] Yu. Karpeshina, On the density of states for the periodic Schrödinger operator, Ark. Mat. 38, 111-137 (2000).

[9] _ Perturbation theory for the Schrödinger operator with a periodic potential, Lecture Notes in Math. vol 1663, Springer Berlin 1997.

[10] T. Kato, Perturbation theory for linear operators, Springer 1966.

[11] E.Korotyaev, A. Pushnitski, On the high energy asymptotics of the integrated density of states, Bull. London Math. Soc. 35, 770-776 (2003).

[12] A.S. Milevskij, Similarity transformations and spectral properties of hypoelliptic pseudodifferential operators on a circle, Funk. Anal. Prilozen. 23 no 3, 71-72 (1989); Engl. transl. in Funct. Anal. Appl. 23 no 3, 231-233 (1989).

[13] _ A simplification of hypoelliptic pseudodifferential operators on the circle via Fourier integral operators, Moscow 1988, dep. in VINITI 05.09.88, No 6856-B88.

[14] A. Mohamed, Asymptotics of the density of states for the Schrödinger operator with periodic electromagnetic potential, J. Math. Phys. 38, 4023-4051 (1997).

[15] L. Parnovski, A.V. Sobolev, Lattice points, perturbation theory and the periodic polyharmonic operator, Ann. H. Poincaré 2, 573-581 (2001).

[16] I. Polterovich, Heat invariants of Riemannian manifolds, Israel J. Math. 119, 239-252 (2000).

[17] M. Reed, B. Simon, Methods of modern mathematical physics, IV, Academic Press, New York, 1975.

[18] G.V. Rozenbljum, Near-similarity of operators and the spectral asymptotic behavior of pseudodifferential operators on the circle, Trudy Moskov. Mat. Obshch. 36, 59-84 (1978) (Russian). 
[19] A.V. Savin, Asymptotic expansion of the density of states for one-dimensional Schrödinger and Dirac operators with almost periodic and random potentials, (in Russian), Sb. Nauchn. Tr. IFTP, 1988, Moscow.

[20] D. Shenk and M. Shubin, Asymptotic expansion of the state density and the spectral function of a Hill operator, Math. USSR Sbornik 56 no. 2, 473-490 (1987).

[21] M.A. Shubin, Weyl's theorem for the Schrödinger operator with an almost periodic potential, Vestnik Moskov. Univ. Ser. I Mat. Mekh. 31, no 2, 84-88 (1976)(Russian). Engl. transl.: Moscow Univ. Math. Bull. 31, 133-137 (1976).

$[22]$, The spectral theory and the index of elliptic operators with almost periodic coefficients, Russian Math. Surveys 34 no 2, 109-157 (1979).

[23] M. Skriganov, Geometrical and arithmetical methods in the spectral theory of the multi-dimensional periodic operators, Proc. Steklov Math. Inst. Vol. 171, 1984.

[24] operator with periodic potential, Inv. Math. 80, 107-121 (1985).

[25] A.V. Sobolev, Asymptotics of the integrated density of states for periodic elliptic pseudo-differential operators in dimension one, to appear in Revista Matematica Iberoamericana (2005).

\author{
Alexander V. Sobolev \\ Department of Mathematics \\ University of Sussex \\ Falmer \\ Brighton BN1 9RH \\ United Kingdom \\ email: A.V.Sobolev@sussex.ac.uk \\ Communicated by Bernard Helffer \\ submitted 30/04/04, accepted 26/07/04
}

\title{
To access this journal online: \\ (20) http://www.birkhauser.ch
}

